\title{
STABILITY OF A FRICTIONAL MATERIAL LAYER RESTING ON A VISCOUS HALF-SPACE
}

\author{
N. TRiantafyllidis \\ Department of Aerospace Engineering. The University of Michigan, Ann Arbor, MI 48109-2 I40, U.S.A. \\ and \\ Y. M. LEROY \\ Shell Research, K.S.E.P.L., 2288 GD Rijswijk Z.H., The Netherlands
}

(Receired 4 March 1993)

\begin{abstract}
THE STABILITY OF a geological two-layer system composed of a frictional material layer of finite thickness, called the overburden, resting on a viscous half-space of lower density is investigated. The salient features of this study are a realistic description of the stiffness of the overburden and its state of (in situ) prestress. and the use of the viscosity of the substratum to define a characteristic time for the stability analysis. A general variational formulation for the lincarized, non-selfadjoint stability problem is presented, followed by asymptotic analyses for the cases of large and small perturbation wavelengths and by an analytical solution in the absence of gravity. Results obtained by a finite-element method are compared with the analytical and asymptotic predictions ; they permit the detection of various modes of instability : interfaceand beam-type modes in the compressive range of deformation, and neck-type modes in the tensile range. It is found that the system's stability is not only governed by geometry and density contrast, as expected from the conclusions of earlier studies on viscous and viscoelastic models, but is also sensitive to the state of in situ stress. A complete parametric study reveals that the overburden material cohesion and workhardening properties have more influence on stability than the friction angle. Furthermore, it is found that critical stresses at neutral stability predicted by deformation theory, which is an appropriate model for studying the initiation of faulting in rocks, are smaller in magnitude than those obtained by the corresponding flow theory with a smooth yield surface. Implications of this work for the interpretation of various laboratory analogue model cxperiments pertaining to geological two-layer systems are also discussed
\end{abstract}

\section{INTRODUCTION}

A VARIETY OF PROBLEMs leads to the question of the precise conditions under which an initially plane interface between two layers of different properties may be destabilized by layer-parallel stresses, counteracted or aided in their effect by body forces. An example that has been of particular interest to geologists and geophysicists has to do with density-stratified two-layer systems in a gravitational field [see, for example, RAMBERG (1981) and references quoted therein]. Earlier theoretical studies of this problem have mostly been concerned with the Rayleigh -Taylor model of two superimposed viscous fluid half-spaces, in which gravity provides the only destabilizing force. In the classical stability analysis of this problem (IAYLOR, IYSO; CHANDRASEKHAR, 1961; DANEŠ, 1964 ; SELIG, 1965) the initial growth of a small interfacial

1993 Shell Research BV. 
perturbation is considered. Although all wavelengths are found to satisfy the linearized perturbation equations, the presence of viscosity implies the existence of a dominant wavelength that possesses the fastest growing amplitude and that is therefore expected to characterize the evolution of the system. However, an essential limitation of fluiddynamic models of interfacial stability has been their inability to account for the effects of layer stiffness and the role of anisotropic and nonhydrostatic states of stress. An alternative approach to the problem of surface as well as interfacial instability in laterally compressed layered media was pioneered by Biot, who introduced viscoelastic constitutive models (BIOT and ODÉ, 1965: BIOT, 1966) and who also treated the onset of interfacial instability as a bifurcation problem (BIOT, 1963a, b, c: 1965). A similar approach was adopted by DORRIS and NEMAT-NASSER (1980) for a problem with a geometry similar to the one considered here: a layer of infinite extent and finite thickness resting on a semi-infinite substratum. However, they modelled the materials in these two regions as elastic or strain-rate-independent elastoplastic with symmetric moduli and disregarded any gradient in in situ stress.

In a recent paper by TRIantafyllidis and LeHnFr (1993) Biot's analysis was extended by considering the combined effects of gravity and nonhomogeneous states of initial stress on the interfacial instability of two half-spaces. In that work the stability of interfacial disturbances of arbitrary wavelength is characterized by means of a perturbation analysis of the system's linearized equations of motion, the time scale for the system being provided by inertia. The stability problem is formulated as a linear (with respect to the square of the stability exponent) eigenvalue problem. A perturbation is considered unstable if its amplitude increases with time and is characterized by a positive value for the square of the stability exponent; periodic perturbations of constant amplitude correspond to a negative value for the square of the stability exponent and are considered stable (given that no time-dependent dissipation mechanism exists in the model). Finally, perturbations that have a zero stability exponent exhibit neutral stability. The corresponding time-independent perturbation modes are shown to be identical to the eigenmodes obtained from a bifurcation analysis of the rate-independent incremental equilibrium equations of the system. quantitatively establishing the connection between the bifurcation and stability formulations of the problem.

Being mainly concerned with the extension of the Taylor model to heterogeneously stressed media, TRIANTAFYLLIDIS and LEHNFR (1993) adopted two simplifying assumptions that limit the application of their work to the study of realistic geological problems. The first assumption, which permitted an analytical solution to the problem, was that the top layer, also termed the overburden, could be taken to be infinitely thick. The second assumption imposed a major symmetry on the incremental moduli of the overburden, limiting their approach to a rather restrictive class of constitutive responses. This constitutive symmetry implied the self-adjoint nature of the eigenvalue problem associated with the linearized stability calculations, which always resulted in real eigenvalues (recall that these eigenvalues coincide with the squares of the stability exponents). The incremental response of geomaterials does not exhibit such constitutive symmetries on account of the frictional nature of their deformation. An immediate consequence of the absence of symmetry is that the linearized stability operator is not self-adjoint, which raises the possibility of complex 
eigenvalues. Moreover, if the characteristic time of the problem is related to inertia, all eigenvalues are squares of the stability exponents and thus flutter-type instabilities are implied when complex eigenvalues are found. Such instabilities, which have not been observed in geological contexts, were mentioned in earlier work by RICE (1976), NeEdLeman (1979) and, more recently, by ChaU and RudNicki (1989) and LoRET and HARIRECHE (1991).

The present study addresses the two important issues circumvented by TRIANTAFYLLIDIS and LEHNER (1993) by generalizing their approach to allow a finite overburden as well as frictional, cohesive-type constitutive responses. This new geometry together with an appropriate description of the in situ stress gradient in the overburden yields a realistic model relevant to the study of layered geological systems and their analogue experiments. Furthermore, the adoption for the first time of a frictional, cohesive-type constitutive model for the overburden will permit the simultaneous study of the onset of folding and faulting in layered geological systems. One of the applications of the present work is the study of the formation and evolution of sedimentary basins.

The paper is organized as follows: Section 2 is devoted to the general formulation of the problem. The novel feature here is that the layers' viscosity and not their inertia provides the time scale relevant for stability. The resulting eigenvalue problem is linear in terms of the stability exponent with a possibility of flutter instability every time a complex eigenvalue with positive real part is found. Finding the stability exponent is considerably simplified (and on the conservative side), since the instantaneous elastic moduli of the bottom layer are disregarded. In addition, the finite size of the overburden makes it possible to account for the effect of erosion and deposition on the top surface of the overburden, which is important for the geological setting of the problem. The erosion condition proposed by BIOT and ODÉ (1965) (named by them the "redistribution" condition), is adopted. Owing to the finite thickness of the overburden, no analytical solution for the layer stability problem is possible for arbitrary thicknesses and in situ stress states. However, one obtains useful asymptotic results for the cases of perturbations with long and short wavelengths compared to the overburden thickness and these results are presented in Section 3. The same section contains the analytical solution that is obtained in the absence of gravity. Results of the numerical, analytical and asymptotic calculations for two types of constitutive laws for pressure-sensitive elastic-plastic geomaterials, one with a smooth yield surface (termed flow-thcory model) and another with vertex effects (tcrmed deformationtheory model) are presented in Section 4 . The main finding, discussed in the final section (Section 5), is the sensitivity of the results to the constitutive properties of the overburden. Another outcome of the calculations is that the stability exponent is always found to be real: a flutter instability is thereby excluded. The concluding section also contains a discussion of laboratory experiments designed as analogue models of geological two-layer systems and suggestions for future work.

\section{Model, Formulation}

The first part of this section is devoted to the formulation of the stability problem for a wide class of constitutive models of the overburden. In the second part we choose 
two specific constitutive equations for the overburden, namely, those expressing the flow and deformation versions of Rudnicki-Rice's model. The second part also includes a description of the in situ stress and the condition of strong ellipticity, whose violation signals the onset of faulting and the limit of validity of our stability analysis.

\subsection{Formulation of the stabilit! problem}

In considering the linearized stability problem for an overburden of thickness $H$ resting atop a semi-infinite substratum, one has to investigate the initial behaviour of the system when subjected, at time $t=0$, to a perturbation of an infinitesimally small amplitude : and arbitrary mode shape about the equilibrium state of interest. The stability investigation is based upon the study of the system's equations of equilibrium, in their rate form. For reasons explained in the Introduction, the inertial terms are distegarded.

In the interest of simplicity, only the plane-strain version of the problem will be analysed here. Material points in the solid are identified by their reference Cartesian coordinates $X_{i}$ in a total Lagrangian formulation or by their current Cartesian coordinates $x_{i}$ in an updated Lagrangian formulation. Note that $x_{i}=X_{i}+u_{i}$, where $u_{i}$ are the corresponding components of the displacement vector from the reference to the current configuration. An updated Lagrangian description of the equations of motion is adopted in which the reference configuration is taken to coincide with the current configuration at time $t=0$, thus modelling the onset of the perturbation from the prestressed equilibrium state whose stability is under investigation.

As shown in Fig. I, the reference configuration of the planar interface between the finite layer and the half-space is taken at $x_{2}=0$, with gravity acting in the

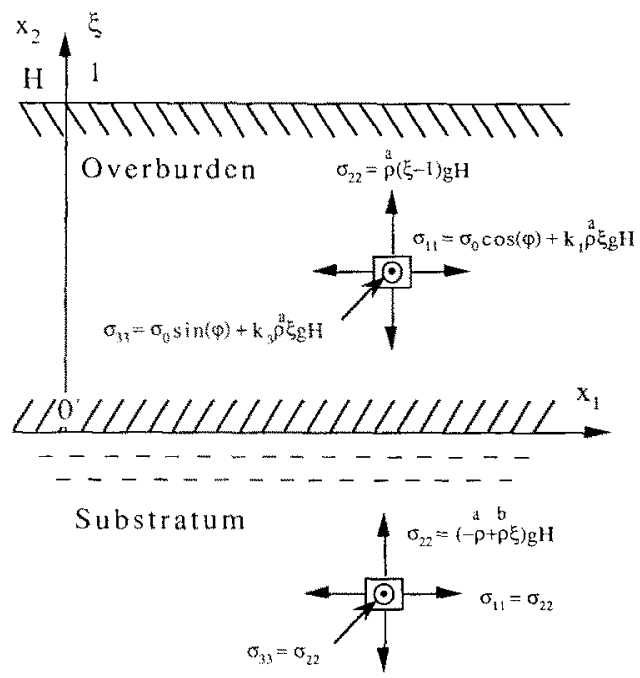

Fri. 1. Geometry of the problem and initiat stress distribution. A layer of pressure-sensitive solid, the overburden. rests on a substratum of viscous luid. The overburden sustains its own weight and a tectonic stress distribution characterized by the stress magnitude parameter $\sigma_{13}$, the gradient parameters $k_{1}$ and $k_{3}$. and the stress orientation angle of. 
$-x_{2}$ direction. Material constants associated with the finite layer are marked by a superscript (") (for "above"), while a superscript (") (for "below") denotes the material constants associated with the semi-infinite substratum.

The rate form of the equations of equilibrium and of the boundary and interface conditions for the system are written as

$$
\begin{aligned}
& \left.\left.\dot{\Pi}_{i, i, i}=0 \quad \forall x_{1} \in\right]-\infty,+\infty\left[, \forall x_{2} \in\right]-\infty, H\right],
\end{aligned}
$$

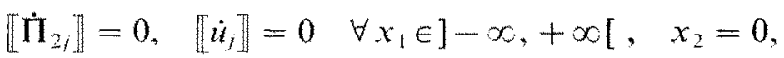

$$
\begin{aligned}
& \left.\dot{\Pi}_{2 j}=\operatorname{sig} \dot{u}_{2} \delta_{j 2} \quad \forall x_{1} \in\right]-\infty,+\infty\left[, \quad x_{2}=H,\right.
\end{aligned}
$$

where $\Pi_{i i}, \beta_{n}, g$ and $\delta_{i j}$ are respectively the components of the first Piola-Kirchhoff stress tensor, the mass density of the overburden, the acceleration of gravity, a scalar that has a value of either 0 or 1 and the Kronecker delta symbol. Differentiations with respect to time and spatial coordinate are denoted by a dot and a comma, respectively. The double brackets I I are defined as the difference between the values of the quantity of interest on the two sides of the interface. The first equation (1) is the incremental equilibrium satisfied at interior points of the two regions considered. The sccond equation, (1) 2 , accounts for traction continuity along the material interface, while the third one, (1) $)_{3}$, expresses the perfect bonding condition at the same interface. Finally, the fourth equation, $(1)_{4}$, accounts for the top surface boundary condition al the overburden. The case $s=1$ corresponds to an "erosion" or "redistribution" condition, according to which any asperity in the top surface is either eroded away if the vertical incremental displacement is positive or flled with sediments of the same material density if this displacement is negative. This redistribution condition. proposed by BIoT and ODE (1965), is translated into the mixed boundary condition (1) $)_{4}$ by prescribing the normal component of the traction to equal the lithostatic pressure of the missing or extra material. Thus, for $s=1$ it is assumed that the characteristic time for erosion or deposition is short compared with the relaxation time of the viscoelastic material in the half-space. For $s=0$ the converse is true : the characteristic time for erosion or deposition in the overburden is long compared with the relaxation time of the viscoelastic material in the half-space, and (1) ${ }_{4}$ corresponds to a traction-free top surface for the overburden.

The system of equations (1) is complemented by the constitutive equations for the two solids:

$$
\begin{aligned}
& \left.\dot{\Pi}_{i i}=\stackrel{u}{L}_{i j k} \dot{u}_{h, k} \quad \forall x_{1} \in\right]-\infty,+\infty\left[, \quad \forall x_{2} \in[0, H],\right. \\
& \left.\left.\dot{\Pi}_{j i}=\stackrel{\mathrm{b}}{L}_{L_{i j k} \mid \dot{u}_{l, k}}+\stackrel{\mathrm{b}}{M}_{i, k} \ddot{u}_{l, k} \quad \forall x_{1} \in\right]-x_{,}+\infty\left[, \quad \forall x_{2} \in\right]-\infty, 0\right] .
\end{aligned}
$$

The overburden is idealized as a rate-independent elastoplastic material. The corresponding incremental moduli $L_{i j k,}$ depend, in general, on a number of material parameters, the current stress state and a set of internal variables that need not be specified further at this point. Geomechanically relevant constitutive models will be considered explicitly later in this section. Note, nevertheless, that frictional materials will be considered for the overburden and that the moduli $L_{i j h}$ will not possess the 
major symmetry: $\stackrel{a}{L}_{i j k l} \neq \stackrel{a}{L}_{k k j i}$ (i.e. the components of the stress-rate tensor $\dot{\Pi}_{i j}$ will not be derivable from a potential function of the deformation gradient rate $\dot{F}_{i j}$ ). The lower half-space is idealized as a nonlinear viscoelastic or viscoplastic material with incremental moduli $\stackrel{L}{L}_{i j k /}$ and instantaneous viscosities $\stackrel{\mathrm{M}}{i j k l}_{\text {; }}$; these depend in general on a number of material parameters, the current stress state and a set of internal variables. Specific forms of this incremental response will be given below in this section and their physical relevance to the stability investigation at hand will be discussed in detail in Section 4. Note again that the only characteristic time of the problem is the relaxation time of the material in the substratum.

Having established the necessary governing equations for the system, we now focus attention on the linearized stability analysis of the problem. Consider the system studied to be at equilibrium and sustaining a given state of prestress, which is assumed to be orthotropic with respect to the coordinate system presented in Fig. 1. The question of how such a state of prestress was achieved through geological times is left open in this paper. All field quantities for the perturbed system are written as a sum of their corresponding unperturbed or fundamental values, which are time independent and marked by the superscript $\left(^{(0}\right)$, and a time-dependent perturbation which is expanded in a power series with respect to the amplitude $:$ of the initial perturbation of the system at time $t=0$. The stress and displacement fields of the perturbed system thus take the form

$$
\begin{aligned}
\Pi_{i j}\left(x_{1}, x_{2}, t\right) & =\prod_{i j}^{0}\left(x_{1}, x_{2}\right)+i \tilde{\Pi}_{i j}\left(x_{1}, x_{2}, t\right)+\Theta\left(\varepsilon^{2}\right), \\
u_{i}\left(x_{1}, x_{2}, t\right) & =\varepsilon \tilde{u}_{i}\left(x_{1}, x_{2}, t\right)+\mathscr{O}\left(\varepsilon^{2}\right) .
\end{aligned}
$$

Following the standard hypothesis adopted in linearized stability analyses, it is assumed that the $c\left(c^{2}\right)$ terms are negligible over the time interval of interest and that the first-order terms adequately characterize the initial evolution of the system. The coeflicients in the system of the linearized equations of motion, derived by introducing (3) into (1) and (2), are independent of time since the moduli in (2) are functions of the time-independent unperturbed state. Consequently, the solutions for the firstorder perturbation stresses $\tilde{\Pi}_{i}$ and displacements $\tilde{u}_{i}$ have the following structure:

$$
\tilde{\Pi}_{i j}\left(x_{1}, x_{2}, t\right)=\exp (\lambda t) \hat{\Pi}_{i j}\left(x_{1}, x_{2}\right) \text { and } \tilde{u}_{i}\left(x_{1}, x_{2}, t\right)=\exp \left(\lambda_{i} t\right) \hat{u}_{i}\left(x_{1}, x_{2}\right) \text {, }
$$

in which the stability exponent $i$ is in general a complex number. The two timeindependent functions $\hat{u}_{i}\left(x_{1}, x_{2}\right)$ and $\hat{\Pi}_{i j}\left(x_{1}, x_{2}\right)$ are the sought eigenmodes and their amplitude remains undetermined by this linear ability analysis. Substituting (4) into (3) and then introducing the result into the equilibrium and constitutive equations (1) and (2), one obtains

$$
\begin{aligned}
& \left.\left(L_{i j k} \hat{u}_{l, k}\right)_{, i}=0 \quad \forall x_{1} \in\right]-\infty .+\infty\left[, \quad \forall x_{2} \in[0, H],\right. \\
& \left.\left.\left.\left.\stackrel{\mathrm{b}}{M}_{i / k i}\right) \hat{u}_{l, k}\right]_{. i}=0 \quad \forall x_{1} \in\right]-\infty,+\infty\left[, \quad \forall x_{2} \in\right]-\infty, 0\right] \text {; } \\
& \left.\left.\stackrel{a}{L}_{i 2 k} \hat{u}_{l, k}=\left(\stackrel{\mathrm{b}}{L_{i 2 k}}+\hat{i}_{M_{i 2 k}}\right) \hat{u}_{l, k} . \quad \| \dot{u}_{i}\right]=0 \quad \forall x_{1} \in\right]-x_{i}+\infty\left[, \quad x_{2}=0\right. \text {, } \\
& \left.\stackrel{a}{L}_{i 2 k} \hat{u}_{i, k}=s \mu g \hat{u}_{2} \dot{\delta}_{i 2} \quad \forall x_{1} \in\right]-\infty,+\infty\left[, \quad x_{2}=H .\right.
\end{aligned}
$$


Note that the time-independent moduli $\stackrel{a}{L}_{i j k l}, \stackrel{\mathrm{b}}{L}_{i j k}, \stackrel{\mathrm{b}}{M}_{i j k /}$ are fully determined by the knowledge of the prestress $\prod_{i j}=\sigma_{i j}$, where $\sigma_{i j}$ denote the components of the Cauchy stress, and the internal variables that are necessary to define the constitutive response of the two materials. These variables will be introduced explicitly at a later stage in this paper.

The above system should be complemented by a decay condition for the mode $\hat{u}_{i}$ away from the interface:

$$
\lim _{x_{2} \rightarrow \infty} \hat{u}_{i}=\lim _{x_{2} \rightarrow+} \hat{u}_{i, j}=0
$$

The interpretation of this condition is that the displacement and the strain at great depth are not changed by the infinitesimal perturbation of the top layer.

In the linear approximation, the stability of the system is governed by the real part of the parameter $\lambda$ which was introduced in (4). Accordingly, if a $\lambda$ is found with a positive real part the equilibrium state of the system is unstable. Conversely, if all solutions of the stability problem require the real part of the parameter $\lambda$ to be negative, the system is said to be stable in the linearized sense, for, according to (4), the perturbation then remains bounded.

The calculation of the stability parameter $\lambda$ is greatly simplified by the additional, and physically reasonable, requirements of material orthotropy with respect to the adopted coordinate system and of translational invariance of the governing equations with respect to the $x_{1}$-direction. Consequently, since there is no shear prestress $\left(\hat{\sigma}_{12}=\hat{\sigma}_{21}=0\right)$, the normal-to-shear coupling components of the moduli $L_{i / k /}, M_{i j /}$ entering the constitutive equations $(2)$ are zero:

$$
L_{1112}=L_{1121}=L_{1211}=L_{2111}=L_{2221}=L_{2212}=L_{2122}=L_{1222}=0 .
$$

Similar relations hold for the moduli $M_{i j k /}$. In addition, the problem's translational invariance in $x_{1}$ implies that: $\partial \sigma_{i j}^{0} / \partial x_{1}=\partial L_{i / k} / \partial x_{1}=\partial M_{i j k} / \partial x_{1}=0$. The superscript $\left({ }^{\circ}\right)$, indicating evaluation of field quantities at $t=0$, is hereafter omitted as superfluous, since all moduli and stresses entering the formulation of the linearized stability problem are evaluated at $t=0$.

In view of the $x_{1}$-invariance of the coefficients $L_{i j k l}$ and $M_{i j k l}$ in (5), the Fourier transform $U_{1}\left(\omega, x_{2}\right)=\mathscr{F}\left[\hat{u}_{1}\left(x_{1}, x_{2}\right) ; x_{1} \rightarrow \omega\right],-\mathrm{i} U_{2}\left(\omega, x_{2}\right)=\mathscr{F}\left[\hat{u}_{2}\left(x_{1}, x_{2}\right) ; x_{1} \rightarrow \omega\right]$ is applied to reduce the system (5) to a set of ordinary differential equations. Consequently,

$$
\begin{aligned}
& \left.-\omega^{2} N_{1111} U_{1}+\omega N_{1122} U_{2.2}+\omega\left(N_{1212} U_{2}\right)_{.2}+\left(N_{1221} U_{1.2}\right)_{.2}=0\right\}
\end{aligned}
$$

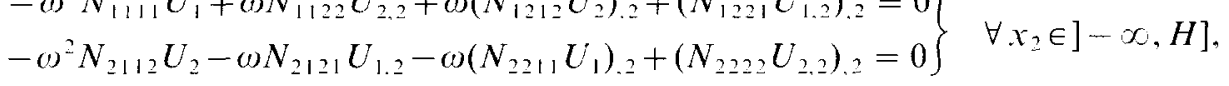

$$
\begin{aligned}
& N_{i j k \prime}\left(x_{2}, \lambda\right) \equiv\left\{\begin{array}{cl}
\stackrel{\mathrm{a}}{L}_{i j k /}\left(x_{2}\right) & \forall x_{2} \in[0, H] \\
\mathrm{b} & \left.\left.\forall x_{2} \in\right]-\infty, 0\right]
\end{array}\right.
\end{aligned}
$$


are the Fourier-transformed equations (5), and (5) . The Fourier transformation of the interface conditions $(5)_{5}$, the boundary conditions $(5)_{4}$ and the decay conditions (6) in the substrate yields:

$$
\begin{aligned}
& \left\{\begin{array}{ll}
(1) N_{1212} U_{2}+N_{1221} U_{12} "=0, & \| U_{1} \rrbracket=0 \\
\left.a N_{221} U_{1}-N_{2222} U_{22}\right\rfloor=0 . & \left.\llbracket U_{2}\right\rfloor=0
\end{array}\right\} \quad x_{2}=0 ; \\
& \left.\begin{array}{c}
\text { (1) } N_{121}, U_{2}+N_{1221} U_{12}=0 \\
N_{2211} U_{1}-N_{2223} U_{22}=-s p g U_{2}
\end{array}\right\} \quad x_{2}=H \text { : } \\
& \lim _{x \rightarrow} U_{i}=\lim _{y,} U_{i_{2}}=0 .
\end{aligned}
$$

The new variable $\xi=x_{2} H$ is now introduced for convenience (see Fig. 1). In view of the $x_{2}$-dependence of the coefficients in the eigenvalue problem given by (7) and (8), a numerical (i.e. a finite-element) method will be employed for its solution.

The weak form of (7) and (8), which forms the starting point for the numerical calculations, is now obtained by respectively multiplying the first and the second differential equation in (7) by the arbitrary functions $\delta U_{1}(\xi)$ and $\delta U_{2}(\xi)$ and subsequently inlegrating by parts in $[0,1]$ while accounling for the interface and boundary conditions (8):

$$
\begin{aligned}
& \int_{11}^{1}\left[\left(N_{1221}^{1} \omega H \mathrm{~d} U_{1}+N_{1212} U_{2}\right) \int_{0 H}^{1} \mathrm{~d} \delta U_{1}+\left(\begin{array}{c}
1 \mathrm{~d} U_{2} \\
-N_{1122}+N_{111} U_{1}
\end{array}\right) \delta U_{1}\right. \\
& +\left(N_{2222} 1 \mathrm{~d} H \mathrm{~d} U_{2}-N_{2211} U_{1}\right){ }_{\omega H}^{1} \mathrm{~d} \delta U_{2}+\left(\begin{array}{cc}
1 & \mathrm{~d} U_{1} \\
& \mathrm{~d} \xi
\end{array}\right.
\end{aligned}
$$

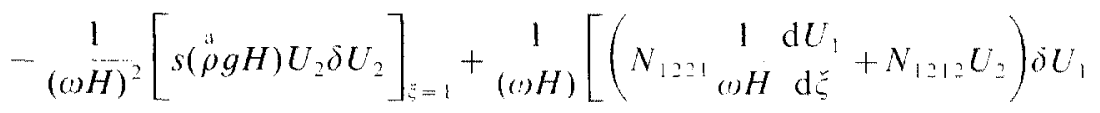

$$
\begin{aligned}
& \left.+\left(N_{2222}(1) \mathrm{d} U_{2}-N_{221} U_{1}\right) \delta U_{2}\right]_{-\infty}=0
\end{aligned}
$$

A word of caution is in order at this point, since $H$ always appears in the denominator of the various terms in (9). The case $b / l=0$, which corresponds to the highly restrictive case of perturbations that are independent of position along the $x_{1}$-direction, is easily analysed directly from (5) and (6). This particular problem, however, is so degenerate that it leaves the stability exponent $i$ indeterminate. It will henceforth be assumed. without loss of generality, that ()$H \neq 0$.

To simplify (9), it is desirable to eliminate the derivative of the eigenmode $U_{i}$ evaluated on the substratum side of the interface. Notice that $\mathrm{d} U_{i} / \mathrm{d} s$ must be related

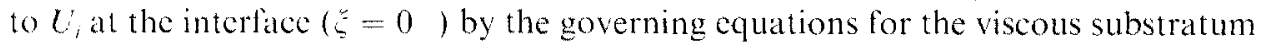
$(\xi 0)$. This relation can be obtained as follows. From the linear governing equations for $U_{i}$ in the half-space (7) and the conditions of decay for $U_{;}$and its gradient $U_{1.2}$ in (8)... one deduces (TRIANTAFYLLIDIS and LFHNIR, 1993) that the desired cigenmode $U_{i}(\zeta)$ for the substratum takes the form: 


$$
U_{i}(\xi)=\sum_{\alpha=1}^{2} \stackrel{x}{V_{i}} \exp \left(z_{\gamma}(\omega) H \xi\right), \quad \xi \leqslant 0, \quad \operatorname{Re}\left(z_{\alpha}\right)>0 .
$$

The constants $V_{i}$ and $z_{\chi}$ satisfy the following linear algebraic system [resulting from the substitution of $(10)$ into $(8)]$ :

$$
\begin{gathered}
\sum_{j=1}^{2} C_{i j}\left(z_{x}, \lambda\right) \stackrel{x}{V_{i}=0} \\
C_{11}\left(z_{x}, \lambda\right) \equiv N_{1,1},(0, \lambda) z_{\gamma}^{2}+\frac{1}{\omega H} \frac{\mathrm{d} N_{1221}}{\mathrm{~d} \xi}(0, \lambda) z_{x}-N_{1111}(0, \lambda) \\
C_{12}\left(z_{x}, \lambda\right) \equiv\left(N_{1,1},(0, \lambda)+N_{1122}(0, \lambda)\right) z_{x}+\frac{1}{\omega H} \mathrm{~d} N_{1212}(0, \lambda) \\
C_{21}\left(z_{x}, \lambda\right) \equiv-\left(N_{21},(0, \lambda)+N_{2211}\left(0^{-}, \lambda\right)\right) z_{x}-\frac{1}{1} H \mathrm{~d} N_{2211}\left(0^{-}, \lambda\right) \\
C_{22}\left(z_{x}, \lambda\right) \equiv N_{2222}(0, \lambda) z_{x}^{2}+\frac{1}{\omega H} \frac{\mathrm{d} N_{2222}}{\mathrm{~d} \xi}(0, \lambda) z_{x}-N_{2112}(0, \lambda) .
\end{gathered}
$$

The linear system (11) has a nontrivial solution for the $V_{i}^{*}$ if its determinant vanishes, a condition that gives $z_{x}$ as the two roots with positive real part of the following fourth-order polynomial in $z_{x}$ :

$$
\operatorname{Det}\left[C_{i j}\left(z_{\gamma}, \lambda\right)\right]=0 \text {. }
$$

We note from the linear system (11) that $\uparrow$

$$
\stackrel{x}{V_{2}}=\gamma_{x} V_{1}, \quad \gamma_{x} \equiv-\begin{aligned}
& C_{11}\left(z_{x}, \lambda\right) \\
& C_{12}\left(z_{x}, \lambda\right)
\end{aligned} .
$$

Using (10) again, we relate the components of the eigenmode $U_{i}$ and their derivatives at $\xi=0$ to the constants $\hat{V}_{i}$ :

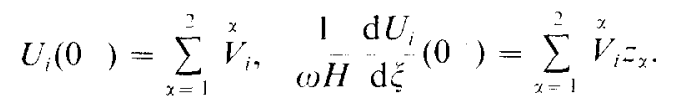

After elimination of these constants, we find the desired relation between the $U_{i}$ and their first derivatives with respect to $\zeta$, evaluated at $\zeta=0$, to be

$$
\begin{aligned}
& \frac{\mathrm{l}}{\omega H} \frac{\mathrm{d} U_{1}}{\mathrm{~d} \xi}(0)=\frac{z_{1} \gamma_{2}-z_{2} \hat{\gamma}_{1}}{\gamma_{2}-\gamma_{1}} U_{1}(0)+\frac{z_{2}-z_{1}}{\gamma_{2}-\gamma_{1}} U_{2}(0), \\
& \omega H \mathrm{~d} \zeta^{2}\left(0^{-}\right)=\frac{z_{1}-z_{2}}{\gamma_{2}-\gamma_{1}} \gamma_{1} \gamma_{2} U_{1}(0)+\frac{z_{2} \gamma_{2}-z_{1} \gamma_{1}}{\gamma_{2}-\gamma_{1}} U_{2}(0) .
\end{aligned}
$$

\footnotetext{
$\uparrow$ The appearance of the same Greek index more than once does not imply summation.
} 
With these new results, the interfacial term in the variational statement (9) now takes the form:

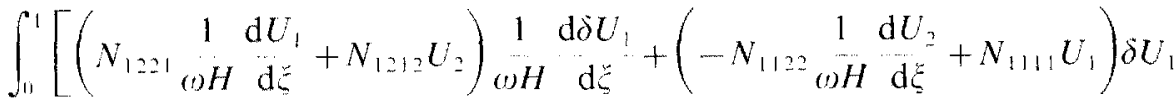

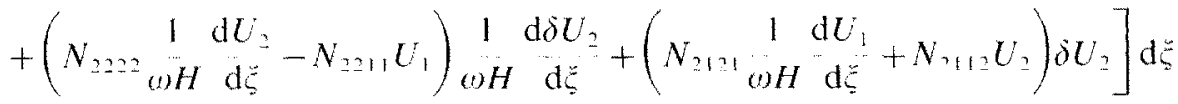

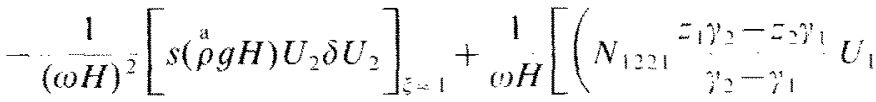

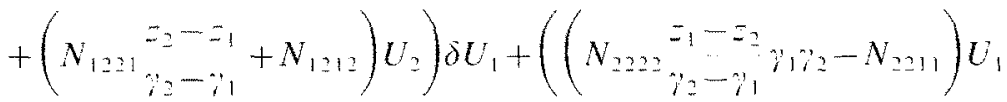

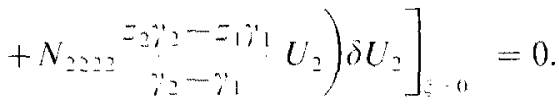

Note that the stability parameter $i$ enters (16) implicitly at the $\xi=0^{\circ}$ interface term in a complicated fashion through the functions $z_{x}$ and $\gamma_{x}$. as may be seen from (10) (12). Solving (16) for the stability parameter $\lambda$ therefore remains difficult unless further simplifying assumptions are made. This is the aim of the remainder of this section.

To begin with, we disregard the instantaneous stiffness of the substratum, thereby idealizing it as an incompressible viscous fluid. This simplification is valid for perturbations that are slow enough as to allow complete relaxation of any nonhydrostatic stresses in the substratum. Assuming that the substratum $\underset{b}{\mathrm{~h}}$ is isotropic and sustains a hydrostatic prestress, the values of the moduli ${\stackrel{\mathrm{b}}{L_{i j k}}}$ and ${\stackrel{\mathrm{h}}{M_{i j k}}}_{\text {entering the definition }}$ (7) of $N_{i, k /}$ are then:

$$
\begin{aligned}
& \sigma_{i i}=(-\stackrel{a}{\rho}+\stackrel{\mathrm{h}}{\rho} \xi) g H \delta_{i, n} \\
& \stackrel{\mathrm{b}}{L}_{i \hbar k}=(\stackrel{\mathrm{a}}{\rho}-\stackrel{\mathrm{b}}{\rho} \xi) g H \delta_{i k} \ddot{\delta}_{i *} \\
& \stackrel{h}{M}_{i j k l}=\stackrel{b}{M}\left[\left(\delta_{i k} \delta_{j l}+\delta_{i l} \delta_{i k}\right)+\frac{1}{\zeta} \delta_{i j} \delta_{k i}\right] \text {. }
\end{aligned}
$$

in which $\stackrel{h}{M}$ and $(\stackrel{h}{M} / \zeta+2 \stackrel{b}{M} / 3)$ are the shear and the bulk viscosities of the half-space, respectively. Introducing (17) into the fourth-order polynomial in $z_{\chi}(12)$, we obtain

$$
\left(z_{\alpha}^{2}-1\right)\left[z_{\alpha}^{2}-z_{x} \stackrel{\stackrel{\rho}{g}}{\omega} \quad \frac{\zeta}{\lambda M+\zeta(2 \lambda \dot{b}+\rho g H)}-1\right]=0 .
$$

Hence. the desired roots of (18) with positive real part are found to be: 


$$
z_{1}=1, \quad z_{2}=1+\zeta \frac{\stackrel{\rho}{\rho g} H}{2(\omega H) \lambda M}+\sigma\left(\zeta^{2}\right)
$$

for $\zeta \ll 1$, i.e. for nearly incompressible behaviour of the viscous substratum.

With this result, and the corresponding expressions for the $\gamma_{x}$ defined in (13) in terms of the $z_{x}$, we use (17) to modify the interface terms of the variational formulation (16). After some rather involved but straightforward algebra, the stability functional in the limiting case of $\zeta \rightarrow 0$ for the incompressible isotropic viscous substratum is obtained:

$$
\begin{aligned}
& \int_{0}^{1}\left[\left(L_{1221} \frac{1}{\omega H} \frac{\mathrm{d} U_{1}}{\mathrm{~d} \xi}+L_{1212} U_{2}\right) \frac{1}{\omega H} \frac{\mathrm{d} \delta U_{1}}{\mathrm{~d} \xi}+\left(\begin{array}{cc}
1 & \mathrm{~d} U_{2} \\
-L_{1122} \omega H & \frac{\mathrm{d} \xi}{\mathrm{d} \xi}+L_{1111} U_{1}
\end{array}\right) \delta U_{1}\right. \\
& \left.+\left(L_{2222} \frac{1}{\omega H} \frac{\mathrm{d} U_{2}}{\mathrm{~d} \xi}-L_{2211} U_{1}\right) \frac{1}{\omega H} \frac{\mathrm{d} \delta U_{2}}{\mathrm{~d} \xi}+\left(\begin{array}{ccc}
1 & \mathrm{~d} U_{1} \\
L_{2121} & \omega H & \frac{\mathrm{d} \xi}{\mathrm{d}}+L_{2112} U_{2}
\end{array}\right) \delta U_{2}\right] \mathrm{d} \xi \\
& -\frac{1}{(\omega H)^{2}}\left[s(\stackrel{a}{\rho} g H) U_{2} \delta U_{2}\right]_{\zeta=1}+\frac{1}{\omega} H\left[\stackrel{a}{\rho} g H\left(U_{1} \delta U_{2}+U_{2} \delta U_{1}\right)+\stackrel{\stackrel{b}{\rho} g H}{\omega H} U_{2} \delta U_{2}\right]_{\zeta=0} \\
& +\Lambda \frac{1}{\omega H}\left[U_{1} \delta U_{1}+U_{2} \delta U_{2}\right]_{\zeta=0}=0
\end{aligned}
$$

where the definition $\Lambda \equiv 2 \lambda \stackrel{\mathrm{b}}{M}$ has been introduced. For simplicity the superscript $\left({ }^{\mathrm{a}}\right)$ for the incremental moduli $L_{i j k}$ is omitted in (20), as it is in the rest of this paper.

Notice from (20) that the stability problem of a rate-independent layer of finite thickness resting on a viscous, isotropic and incompressible infinite substratum has been formulated as a linear eigenvalue problem for the stability exponent $\lambda$. Moreover, the fact that the eigenvalue $\Lambda(=2 \lambda M)$ appears only in terms that are evaluated on the interface $(\xi=0)$ results in an attractively simple method to calculate $\Lambda$ (as an eigenvalue of a $2 \times 2$ matrix), as is outlined in Subsection 4.1 .

\subsection{Constitutive model, in situ stress and condition of strong ellipticity}

Having established the general variational formulation for the stability of a finitethickness layer resting on an incompressible viscous fluid, we now need to specify the constitutive propertics of the overburden and the state of prestress cxisting in the system. We also set up the strong ellipticity condition that determines the limit of validity of our stability analysis.

The general structure of the incremental moduli $L_{i j k}$ introduced in (2) is

$$
L_{i j k l}=\mathscr{L}_{i j k l}-\frac{1}{2}\left(\sigma_{i k} \delta_{j l}+\sigma_{i l} \delta_{j k}\right)+\frac{1}{2}\left(\sigma_{j k} \delta_{i l}-\sigma_{j l} \delta_{i k}\right) .
$$

The incremental moduli $\mathscr{L}_{i j k l}$ relate the Jaumann rate $t_{i j}$ of the Kirchhoff stress to the rate-of-deformation tensor $D_{k l}: \stackrel{\nabla}{\tau}_{i j}=\mathscr{L}_{i j k l} D_{k i}$. The constitutive response of the overburden is assumed to be that of a frictional geomaterial model of the type 
introduced by RUDNicki and Rice (1975). Their model, based upon flow theory with a smooth yield surface. yields the following expression for the moduli $\mathscr{L}_{i i k}$ :

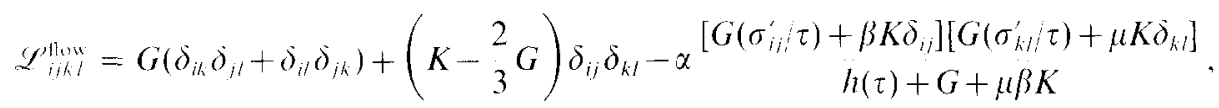

where $G$ and $K$ are, respectively, the elastic shear and bulk moduli of the material, $\beta$ the dilatancy parameter, $\mu$ the friction coefficient; and $\alpha$ a scalar that has a value of 0 or 1 . Because we have chosen an isotropic hardening model, the hardening coefficient $h$ in (22) is related solely to the equivalent shear stress $\tau$. The isotropic yield function depends on a single internal variable that is identified with the accumulated plastic strain $;^{, p}$ and is written as $F\left(\sigma_{i}, i^{p}\right) \equiv \tau+\mu p-f\left(; i^{p}\right) \leqslant 0$. The equivalent shear stress $\tau$ and the equivalent pressure $p$ are related to the stresses $\sigma_{i \prime}$ by

$$
\tau=\left(\frac{1}{2} \sigma_{i j}^{\prime} \sigma_{i j}^{\prime}\right)^{1 / 2}, \quad p=\frac{\sigma_{k k}}{3}, \quad \sigma_{i j}^{\prime}=\sigma_{i j}-p \delta_{i j} .
$$

The value of the coefficient $g$. in (22) depends on the usual loading $(F=0, \dot{F}=0$ for which $x=1$ ) unloading $(F=0, \dot{F}<0$ or $F<0$ for which $x=0$ ) conditions. In addition, the hardening coefficient $h$ is related to the radius of the elastic domain in stress space $f\left(;^{\circ}\right)$ by

$$
h=\frac{\mathrm{d} f}{\mathrm{~d}, n^{\prime \prime}}
$$

The function $f\left(x^{i}\right)$ can be determined from the knowledge of the material's uniaxial stress-strain curve. Specific choices of uniaxial stress strain curves will be made for the subsequent numerical calculations.

The moduli (22) of the isotropic flow theory imply an unrealistically stiff response to sudden changes in the loading path in the plastic domain. This deficiency is the reason that the flow theory model overpredicts the critical load for the localization of deformation. To remedy this shortcoming. RUINICKI and RICE (1975) also proposed a deformation-theory version of (22) whose incremental moduli are given by:

$$
\begin{aligned}
& p^{\prime \prime / h /}=G^{\mathrm{d}}\left(\delta_{i k} \delta_{i t}+\delta_{i j} \delta_{j h}\right)+\left(K^{\mathrm{d}}-\frac{2}{3} G^{\mathrm{d}}\right) \delta_{i j} \delta_{k l} \\
& -\varkappa^{\left[G^{\mathrm{d}}\left(\sigma_{i / /}^{\prime} / \tau\right)+\beta^{\mathrm{d}} K^{\mathrm{d}} \delta_{i j}\right]\left[G^{\mathrm{d}}\left(\sigma_{k / /} / \tau\right)+\mu^{\mathrm{d}} K^{\mathrm{d}} \delta_{k l}\right]} ; \\
& \underset{G^{\mathrm{d}}}{1}=\frac{1}{G}+\frac{1}{h_{1},} \quad \underset{K^{\mathrm{d}}}{1}=\frac{1}{K}-\underset{\left(h_{1}-h\right)}{B \mu} \quad \begin{array}{c}
1 \\
h^{\mathrm{d}}
\end{array}=\frac{1}{h}-\frac{1}{h_{!}} . \\
& \frac{1}{\beta^{\mathrm{d}}}=\frac{1}{\beta}\left(1-\frac{h}{h_{1}}\right), \quad \frac{1}{\mu^{\mathrm{d}}}=\frac{1}{\mu}\left(1-\frac{h}{h_{1}}\right) .
\end{aligned}
$$

where the hardening coefficient $h$ is still given by $(24)$ and the secant coefficient $h_{1}$ is given in terms of the accumulated plastic stratin ${ }^{\prime \prime}$ by 


$$
h_{1}=\stackrel{\tau}{i, p} \text {. }
$$

The reader interested in the derivations of the deformation and flow-theory moduli of Rudnicki-Rice's model is referred to the original work (RUDNICKI and RICE., 1975). The expressions for the incremental moduli in terms of the existing prestress state in the overburden and its material constants have been recorded here only for completeness of the presentation.

We now specify the state of prestress in the finite layer $(\xi \in[0,1])$ in terms of the four parameters $\sigma_{0}, \varphi, k_{1}$ and $k_{3}$ :

$$
\begin{gathered}
\sigma_{11}(\xi)=\sigma_{0} \cos (\varphi)+k_{1} \rho \dot{a} g H \xi, \quad \sigma_{22}(\xi)=\stackrel{a}{\rho} g H(\xi-1), \\
\sigma_{33}(\xi)=\sigma_{0} \sin (\varphi)+k_{3} \rho g h \xi .
\end{gathered}
$$

The first parameter, $\sigma_{0}$, characterizes the magnitude of the in-plane stresses in the overburden at the interface level. The second parameter, $\varphi$, defines the orientation of these stresses with respect to the coordinate system adopted. Finally, the remaining two parameters, $k_{1}$ and $k_{3}$, provide information on the prestress gradient in the overburden along the $x_{1}$ and $x_{3}$ directions, respectively. In geological applications the values of these stress gradient parameters lie in the interval $[0,1]$.

In the stability analysis based on (20) attention is restricted to (sufficiently smooth) global-type modes $\hat{\imath}_{i}$. Hence, one must verify that the stress state in the overburden is such that initiation of any local type of instability in the form of a shear band, i.e. faulting, is precluded. Consequently, the stability calculations will apply only to those stress states that satisfy the strong ellipticity condition for all the points in the overburden, namely (see RicE, 1976),

$$
L_{i j k}(\zeta) m_{i} n_{j} n_{k} m_{l}>0, \quad \forall \mathbf{m}, \mathbf{n} \in \mathbb{R}^{3}(\|\mathbf{m}\|=\|\mathbf{n}\|=1), \quad \forall \xi \in[0,1] .
$$

$\Lambda$ condition equivalent to (28) requires that the matrix $L_{i j k}(\xi) n_{j} n_{k}$ be positive definite for all three-dimensional unit vectors $\mathbf{n}$, i.e. $\forall \mathbf{n} \in \mathbb{R}^{3}$. After some straightforward manipulations, this yields the following inequalities that have to be satisfied by $L_{i / h /}(\xi)$ in the interval $[0,1]$ :

$$
\begin{gathered}
L_{1111}(\xi), \quad L_{1221}(\xi), \quad L_{2112}(\xi), \quad L_{2222}(\xi)>0 \\
\left(L_{1122}(\xi)+L_{1212}(\xi)\right)\left(L_{2211}(\xi)+L_{2121}(\xi)\right) \leqslant\left[\left(L_{1221}(\xi) L_{2112}(\xi)\right)^{1 / 2}\right. \\
\left.+\left(L_{1111}(\xi) L_{2222}(\xi)\right)^{12}\right]^{2}
\end{gathered}
$$

Whenever these conditions, which depend on the imposed stress state on the layer, are satisfied, the appearance of a shear-band-type instability is excluded. The neutral stability curves presented in Section 4 terminate at points where (29) is first violated, thus signaling the onset of faulting as the dominant type of instability.

It should also be repeated at this point that the statement of the stability problem (20), (21), (27) and ellipticity conditions (28) is valid for a wide class of constitutive relations, as long as these can be cast in the incremental form (2). The present choice of the incremental moduli, in accord with the models proposed by Rudnicki and Rice, 
does not restrict the generality of the formulation of the stability problem given in this section.

\section{Spectal Cases}

The results of our study are presented in the form of stability diagrams depicting stable and unstable regions in a plot of dimensionless wave number $(\omega H)$ versus a dimensionless stress $\left(\sigma_{0}, G\right)$. The curves separating stable from unstable regions mark the dimensionless critical wave number at neutral stability $(\omega H)$ c as a function of the stress state imposed on the finite layer. In the absence of gravity, these curves are found analytically. In the presence of gravity, two such stability diagrams have to be presented for each given set of material properties in the overburden. The necessity of introducing two diagrams will become clear during the presentation of the asymptotic analyses and is essentially due to the presence of a gradient in in situ stress. The first diagram corresponds to a given layer thickness, i.e. to a fixed dimensionless quantity $\Delta \rho g H / G$, and provides the critical wavelength $L_{c}=2 \pi H /(\omega H)_{c}$ corresponding to a known stress state in the layer. The second diagram provides the critical height of the layer $H_{\mathrm{c}}=(\omega H)_{\mathrm{c}}(\omega$, which is the height required for the stability transition of the finite layer at a given perturbation wavelength $L=2 \pi /($ ). i.e. for a fixed dimensionless quantity $\wedge \rho g / G(1)$, corresponding to a known stress state in the layer. Henceforth, $\Delta \rho \equiv \stackrel{a}{\rho}-\stackrel{b}{\rho}$ denotes the density difference between the overburden and the substratum.

Although the calculation of the above-mentioned stability diagrams is based on a numerical technique. i.e. a finite-element discretization of the stability functional in (20), a number of special cases can be solved analytically, thus providing valuable insight into the problem as well as an independent verification of the numerical results. The asymptotic restilts are derived for the two possible limiting cases for the wavelength of the disturbance with respect to the layer's thickness, namely, the long-wavelength limit $(\omega H \rightarrow 0)$ and the short-wavelength limit $(\omega H \rightarrow \infty)$. Each limit is studied for the two different stability calculations, one for the fixed overburden layer height $(\Delta \rho g H / G=$ const) and the other for the fixed wavelength disturbance $\left(\Delta \rho g_{i}(G(1)=\right.$ const $)$. Finally, the stability results in the absence of gravity $(\Delta \rho g H / G=\Delta \rho g / G(t)=0)$, which can be found analytically, are also presented in this section.

Readers interested primarily in the numerical results of this investigation can find the main conclusions of this section in Subsection 3.4 and then proceed directly to Section 4.

\subsection{Long-wavelength limit (o) $H$, 0)}

The investigation of the stability of our geological structure to long-wavelength perturbations yields the stability exponent along the horizontal axis of the $(a H)$ versus $\left(\sigma_{0} / G\right)$ plots presented in the next section. We also construct an asymptotic expression for the neutral stability curves for the case of constant $\Delta \rho g / G(\omega$. The combined knowledge of the neutral stability curves and the stability exponent along 
the horizonal axis $(\omega H \rightarrow 0)$ permit the identification of the stability regions of the graphs. The asymptotic expansion of the neutral stability curves, simplified for the special case of a linear elastic response of the overburden, is discussed and compared with earlier results obtained by RAMBERG and STEPHANSSON (1964).

3.1.1. Fixed layer thickness ( $\Delta \rho g H / G=$ const). The starting point for the stability analysis is the variational formulation (20) of the problem, with the incremental moduli of the overburden $L_{i j k i}$ given by (21)-(26) and the corresponding stresses by (27). For simplicity, the small parameter $\varepsilon \equiv \omega H$ is introduced, which permits (20) to be rewritten as

$$
\begin{aligned}
& \int_{\theta}\left[\left(L_{1221} \frac{1}{\varepsilon} \frac{\mathrm{d} U_{1}}{\mathrm{~d} \xi}+L_{1212} U_{2}\right) \frac{1 \mathrm{~d} \delta U_{1}}{\varepsilon}+\left(-L_{1122} \frac{1 \mathrm{~d} U_{2}}{\mathrm{~d} \xi}+L_{111} U_{1}\right) \delta U_{1}\right. \\
& \left.+\left(L_{2222} \frac{1}{\varepsilon} \frac{\mathrm{d} U_{2}}{\mathrm{~d} \xi}-L_{2211} U_{1}\right) \frac{1 \mathrm{~d} \delta U_{2}}{\varepsilon}+\left(L_{2121} \frac{1 \mathrm{~d} U_{1}}{\mathrm{~d} \xi}+L_{2112} U_{2}\right) \delta U_{2}\right] \mathrm{d} \xi \\
& \quad-\left[\frac{1}{\varepsilon^{2}} \operatorname{s} \rho g H U_{2} \delta U_{2}\right]_{\xi=1}+\left[\frac{1}{\varepsilon} \rho g H\left(U_{1} \delta U_{2}+U_{2} \delta U_{1}\right)+\frac{1}{\varepsilon^{2}} \rho g H U_{2} \delta U_{2}\right. \\
& \left.+\frac{\Lambda}{\varepsilon}\left(U_{1} \delta U_{1}+U_{2} \delta U_{2}\right)\right]_{\xi=0}=0 .
\end{aligned}
$$

An inspection of (30) suggests the following asymptotic expansions for $U_{1}, U_{2}$ and $\Lambda$ in terms of $\varepsilon$ :

$$
\begin{aligned}
U_{i}(\xi, \varepsilon) & =\mathscr{U}_{i}(\xi)+\varepsilon \dot{U}_{i}(\xi)+c\left(\varepsilon^{2}\right), \\
\Lambda & =\varepsilon^{-1} \Lambda_{-1}+\Lambda_{0}+\varepsilon \Lambda_{1}+\sigma\left(\varepsilon^{2}\right)
\end{aligned}
$$

Note that the stress components and the incremental moduli depend on the vertical position $\xi$ in the overburden [see (21) -(27)]; however, they are independent of the dimensionless parameter $\varepsilon$.

Various choices are possible to fix the amplitude of the eigenmode $U_{i}$; the following normalization condition is assumed:

$$
\int_{0}^{1}\left[U_{1} U_{1}+\stackrel{0}{U_{2}} U_{2}\right] d \xi=1
$$

Substitution of the asymptotic expressions (31) into (30), subsequent expansion in terms of ascending powers of $\varepsilon$ and grouping of terms of the same order in $\varepsilon$ results in the following expression for the term of lowest order:

$$
\begin{aligned}
& C\left(\varepsilon^{-2}\right): \int_{0}^{1}\left[\left(L_{1221} \frac{\mathrm{d} U_{1}}{\mathrm{~d} \xi}\right) \frac{\mathrm{d} \delta U_{1}}{\mathrm{~d} \xi}+\left(L_{2222} \frac{\mathrm{d} \tilde{U}_{2}}{\mathrm{~d} \xi}\right) \frac{\mathrm{d} \delta U_{2}}{\mathrm{~d} \xi}\right] \mathrm{d} \xi-\left[\stackrel{\mathrm{a}}{s \rho g H \dot{U}_{2} \delta U_{2}}\right]_{\xi=1} \\
& +\left[\stackrel{\mathrm{b}}{\rho} g H \ddot{o}_{2} \delta U_{2}+\Lambda_{-1}\left(\stackrel{0}{U_{1}} \delta U_{1}+\stackrel{0}{U_{2}} \delta U_{2}\right)\right]_{\xi=0}=0
\end{aligned}
$$

Upon integration of (33) by parts, and in view of the arbitrariness of $\delta U_{1}(9)$ and 
$\delta U_{2}(5)$. one arrives at two two-point boundary-value problems. The first of the two. corresponding to $\delta U_{1}(\zeta)$. is

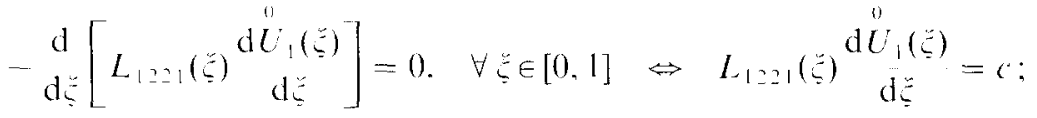

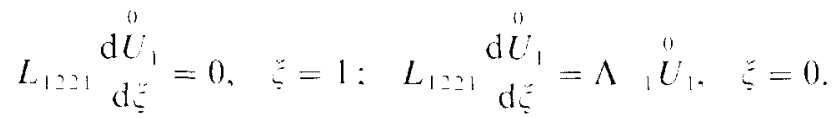

The constant $"$ has to equal zero, since $L_{121}(\zeta)$ differs from zero [the overburden is assumed to be in the elliptic range. see (29)]. Consequently, one finds that $U_{,}(\xi)$ is a constant, denoted here by $U_{1}$, and the complete solution of (34) reads

$$
\ddot{U}_{1}^{\prime}(\zeta)=\ddot{U}_{1} . \quad \forall \check{G} \in[0.1]: \Lambda, \ddot{U}_{1}=0 .
$$

The second two-point value problem, corresponding to $\delta C_{2}(\zeta)$ in (33), is

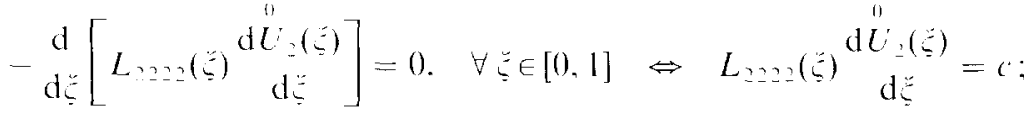

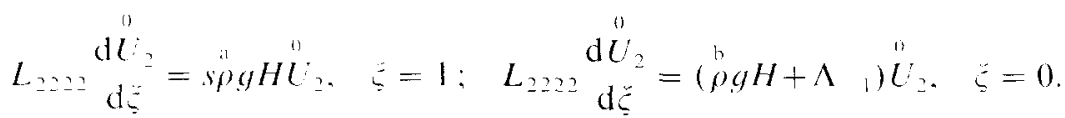

The differential equation (36), implies that $\mathrm{d} U_{2}(\zeta) \mathrm{d}=L_{2}=L_{222}(\xi)$. Note that $L_{2222}$ differs from zero in view of the ellipticity condition (29), while the two boundary conditions (36), dictate the value of the constant a to be spgH $L_{2}(1)-$

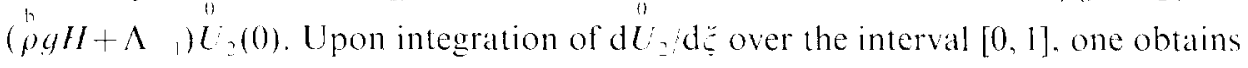

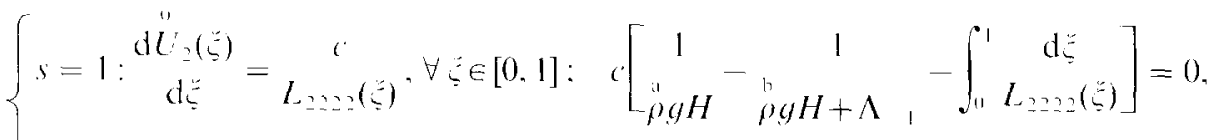

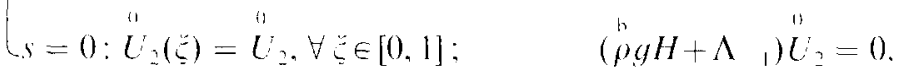

Stability depends on the sign of the real part of $\Lambda$, which we now determine. For the case in which material in the overburden is heavier than that in the substream $(p) p)$, one distinguishes, according to the results in (35) and (37), the following two cases:

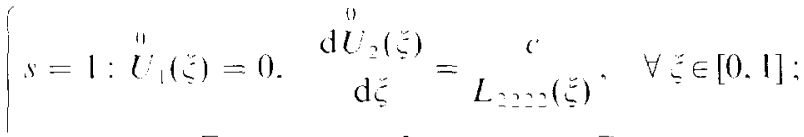

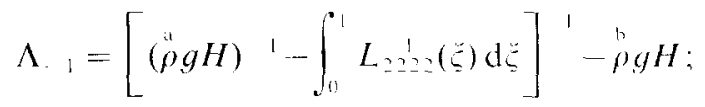

$$
\begin{aligned}
& x=0: U_{1}(\xi)=U_{1} . \quad U_{2}(\xi)=0, \quad \forall \xi \in[0,1] ; \quad \Lambda \quad=0 .
\end{aligned}
$$

where $c$ is nonzero it there is erosion $(s=1)$, otherwise ${ }^{0} t_{1} \neq 0$. The physical interpretation of the above stability result is straightforward. When erosion is accounted for 
$(s=1)$, the stability to long-wavelength peturbations depends on the stress state in the top layer, as can be seen from (38). From (21) and (27), it is known that the modulus $L_{2222}=\mathscr{L}_{2222}+\stackrel{a}{\rho} g H(1-\xi)$; and since $\mathscr{L}_{2222}(\xi)>\stackrel{a}{\rho} g H \xi$ for the constitutive models considered here, one easily verifies that $\stackrel{a}{\rho} g H \int_{0}^{1} L_{2222}(\xi) \mathrm{d} \xi<1$. This line of reasoning leads to the conclusion that $\Lambda_{-1}>\Delta \rho g H>0$. The other possibility given in (35) is that $\Lambda_{-1}=0$ and thus justifies the choice made in (38).

We conclude that the layer of finite thickness, for the case of erosion on its top surface, is unstable with respect to long-wavelength perturbations regardless of the prevailing stress state. This situation is easily interpreted as the well-known instability of an interface between two fluids if the heavier (of density $\stackrel{i t}{\rho}$ ) lies on top of the lighter one (of density $\stackrel{b}{\rho}$ ).

In the absence of erosion on the top surface $(s=0)$, one sees from (38) that $\Lambda_{1}=0$. The second possibility indicated in $(37)\left(\Lambda_{1}=-\stackrel{a}{\rho} g H<0\right)$ implies a smaller real part for $\Lambda$, thus explaining the choice made in (38). The determination of the overburden stability will therefore require finding the first nonzero term in the expansion of $\Lambda$ in $(31)_{2}$. To this end, one notices from the structure of (30) that the odd terms in the 8 expansion of $U_{1}$ and the even terms in the $s_{1}$ expansions of $U_{2}$ and of $\Lambda$ are zero:

$$
\stackrel{2 k+1}{U_{1}}(\xi)=\stackrel{2 k}{U},(\check{\zeta})=\Lambda_{2 k}=0, \quad \forall k \varepsilon \mathbb{N} .
$$

The first term that could provide information on the stability of our system with respect to long-wavelength perturbations and in the absence of erosion is thus $\Lambda_{1}$. We now proceed to its determination. From the inclusion of (39) in the asymptotic expansions (31) and their subsequent substitution into (30), one obtains for the nextorder term

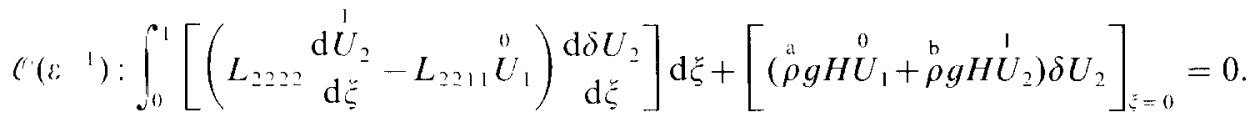

Upon integration of (40) by parts, and in view of the arbitrariness of $\delta U_{2}(\xi)$, one obtains the following differential equation and boundary conditions :

$$
\begin{aligned}
& -\underset{\mathrm{d} \xi}{\mathrm{d}}\left[L_{2222}(\xi) \frac{\mathrm{d} \dot{U}_{2}(\xi)}{\mathrm{d} \xi}-L_{2211}(\xi) \stackrel{0}{U_{1}}\right]=0, \quad \forall \xi \in[0,1]
\end{aligned}
$$

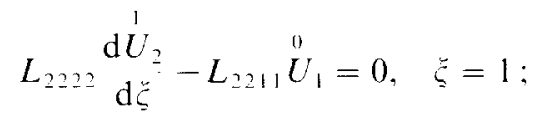

$$
\begin{aligned}
& L_{2222} \stackrel{\mathrm{d} U_{2}}{\mathrm{~d} \xi}-L_{2211} \stackrel{0}{U_{1}}=\stackrel{\mathrm{a}}{\rho} g H \stackrel{0}{U}_{1}+\stackrel{\mathrm{b}}{\rho g} H \stackrel{\mathrm{I}}{U}_{2}, \quad \xi=0 .
\end{aligned}
$$

After simplification, these equations read:

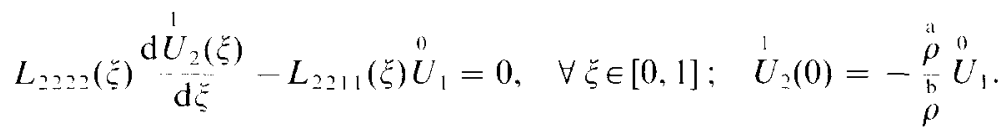


Continuing in a similar way with the $(1)$ term of the asymptotic expansion of (30), we obtain

$$
\begin{aligned}
\mathscr{C}(1): \int_{0}^{1}\left[\left(L_{1221} \frac{\mathrm{d} \dot{U}_{1}}{\mathrm{~d}}+L_{1212} \stackrel{1}{U_{2}}\right) \frac{\mathrm{d} \delta U_{1}}{\mathrm{~d} \xi}\right. & \left.+\left(-L_{1122} \frac{\mathrm{d} U_{2}}{\mathrm{~d} \xi}+L_{1111} \stackrel{0}{U_{1}}\right) \delta U_{1}\right] \mathrm{d} \xi \\
& +\left[\left(\stackrel{\mathrm{a}}{\rho} g H \stackrel{1}{U}_{2}+\Lambda_{1} \stackrel{0}{U}_{1}\right) \delta U_{1}\right]_{\xi=0}=0
\end{aligned}
$$

which in view of the arbitrariness of $\delta U_{1}(\xi)$ gives, upon integration by parts,

$$
\begin{aligned}
& -\frac{\mathrm{d}}{\mathrm{d} \xi}\left[L_{1221}(\xi) \frac{\mathrm{d} \dot{U}_{1}(\xi)}{\mathrm{d} \xi}+L_{1212}(\xi) U_{2}(\xi)\right]+\left[-L_{1122}(\xi) \frac{\mathrm{d} U_{2}(\xi)}{\mathrm{d} \xi}+L_{1111}(\xi) U_{1}\right]=0 \\
& \forall \xi \in[0,1] \text {; } \\
& L_{1221} \frac{\mathrm{d}^{2}}{\mathrm{~d}} \stackrel{1}{\xi}+L_{1212} \stackrel{1}{U}_{2}=0, \quad \xi=1 \\
& L_{12121} \frac{\stackrel{2}{U}^{\mathrm{d}}}{\mathrm{d}}+L_{1212} \stackrel{1}{U}_{2}=\stackrel{n}{\rho} g H \stackrel{1}{U}_{2}+\Lambda_{1} \stackrel{0}{U}_{1}, \quad \xi=0 \text {. }
\end{aligned}
$$

The above system is of the form $-\mathrm{d} f / \mathrm{d} \xi+g=0$ for $\xi \in[0,1]$, with $f(0)=a$ and $f(1)=b$. To permit a solution, the function $g(\xi)$ and the scalars $a$ and $b$ have to satisfy the compatibility condition $\int_{0}^{l} g(\xi) \mathrm{d} \xi=b-a$. Consequently, from (44) and (42), one obtains

$$
\Lambda_{1}=\frac{\stackrel{\mathrm{\rho}}{\mathrm{b}}}{\rho}(\stackrel{\mathrm{a}}{\rho} \mathrm{g} g H)-\int_{0}^{1}\left(L_{1111}-\frac{L_{1122} L_{2211}}{L_{2222}}\right) d \xi,
$$

if one uses the fact that $\stackrel{0}{U}_{1}$ differs from zero [see (38)] for $s=0$.

We have in (45) an expression for the leading term of the asymptotic development (31), whose sign determines the stability of our system with respect to long-wavelength perturbations in the absence of erosion. A physical interpretation of the integral in (45) can be given for the case of plane-strain uniform stretching of the overburden under plane-stress conditions (i.e. $\dot{\Pi}_{22}=0$ ). In such a case $(2)_{1}$ gives $\dot{\Pi}_{11}=E_{1} \dot{u}_{1,1}$, in which $E_{i} \equiv L_{1111}-L_{1122} L_{2211} / L_{2222}$ is the tangent modulus relating the first Piola Kirchhoff stress rate to the corresponding strain rate in the direction of stretching. This modulus is in fact the integrand of (45). For the uniform stretching case at hand, the integral $\left(\int_{0}^{1} E_{,} \mathrm{d} \xi\right)$ in the right-hand side of (45) thus represents the slope of the force displacement response of the layer. Obviously, in the absence of gravity, stability is ensured for positive slope, $\int_{0}^{1} E_{t} \mathrm{~d} \xi>0$, as one can see from (45). We thus conclude that, in the absence of erosion $(s=0)$, as long as the slope of the force-displacement curve for the plane-stress uniaxial stretching of the layer is larger than the lithostatic pressure at the interface [for $(\stackrel{a}{\rho} / \stackrel{b}{\rho}) \approx 1]$, the overburden is stable with respect to longwavelength perturbations. 
3.1.2. Perturbation with fixed wavelength $(\Delta \rho g / G \omega=$ const). Again, the starting point for the analysis is the variational formulation (20) with the incremental moduli for the top layer $L_{i j k}$ given by (21)-(26) and the corresponding stresses by (27). Using, as before, the same definition for the small parameter $\varepsilon \equiv \omega H$, one obtains from (20)

$$
\begin{aligned}
& \int_{0}^{1}\left[\left(L_{1221} \frac{1-\frac{\mathrm{d} U_{1}}{\varepsilon}}{\mathrm{d} \xi}+L_{1212} U_{2}\right) \frac{1 \mathrm{~d} \delta U_{1}}{\varepsilon}+\frac{1}{\mathrm{~d} \xi}+\left(-L_{1122} \frac{1 \mathrm{~d} U_{2}}{\varepsilon}+L_{1111} U_{1}\right) \delta U_{1}\right. \\
& \left.+\left(L_{2222} \frac{1}{\varepsilon} \frac{\mathrm{d} U_{2}}{\mathrm{~d} \xi}-L_{2211} U_{1}\right) \frac{1 \mathrm{~d} \delta U_{2}}{\varepsilon}+\left(L_{2121} \frac{1}{\varepsilon} \frac{\mathrm{d} U_{1}}{\mathrm{~d} \xi}+L_{2112} U_{2}\right) \delta U_{2}\right] \mathrm{d} \xi \\
& +\left[\frac{1}{\varepsilon} s \stackrel{\stackrel{\mathrm{a}}{\rho} g}{\omega} U_{2} \delta U_{2}\right]_{\xi=1}+\left[\frac{\stackrel{\mathrm{a} g}{\omega}}{\omega}\left(U_{1} \delta U_{2}+U_{2} \delta U_{1}\right)+\frac{1}{\varepsilon} \frac{\stackrel{\mathrm{b}}{\rho}}{\omega} U_{2} \delta U_{2}\right. \\
& \left.+{ }^{\Lambda}\left(U_{1} \delta U_{1}+U_{2} \delta U_{2}\right)\right]_{i=0}=0
\end{aligned}
$$

which differs from (30) in its boundary terms.

The asymptotic expansions for $U_{1}, U_{2}$ and $\Lambda$ are still given by (31), with $\Lambda \quad=0$, and the mode normalization condition (32). However, it follows from the expressions for the in situ stress (27) that those do depend now on the small parameter $\varepsilon$ :

$\sigma_{11}=\sigma_{0} \cos (\varphi)+\varepsilon k_{1} \stackrel{\stackrel{\mathrm{a}}{\rho g}}{\frac{(}{()}} \xi, \quad \sigma_{22}=\varepsilon \frac{\stackrel{\mathrm{a}}{\rho g}}{(t)}(\xi-1), \quad \sigma_{33}=\sigma_{0} \sin (\varphi)+\varepsilon k_{3} \frac{\stackrel{\mathrm{a}}{\rho} \frac{g}{(l)}}{\xi}$.

As can be seen from the expressions (21)-(26) for $L_{i j k i}$, the incremental moduli are functions of the stress state. Consequently, according to (47), they should obey the following asymptotic expansion :

$$
L_{i j k}(\xi, \varepsilon)=\stackrel{0}{L}_{i j k l}+\varepsilon \stackrel{1}{L}_{i j k /}(\xi)+\varepsilon^{2} L_{i j k /}(\xi)+\mathscr{C}\left(\varepsilon^{3}\right) .
$$

Notice that the $\xi$-independence of the zeroth-order term in $\varepsilon$ of the stresses given in (47) is shared by the zeroth-order term in the expansion of the incremental moduli $\stackrel{0}{i j k i}_{i}$ in (48).

A substitution of the asymptotic expressions (31) and (48) into (46), an expansion in terms of ascending powers of $\varepsilon$ and a grouping of the terms of the same order in $\varepsilon$ give the following results for the term of lowest order:

$$
\mathscr{U}\left(\varepsilon^{2}\right): \int_{0}^{1}\left[\left(\begin{array}{ll}
0 & \frac{0}{U_{1}} \\
L_{1221} & \frac{\mathrm{d} \delta U_{1}}{\mathrm{~d} \xi}
\end{array}\right)\left(\begin{array}{ll}
0 & \frac{0}{U^{U}} \\
L_{2222} & \frac{\mathrm{d} \delta U_{2}}{\mathrm{~d} \xi}
\end{array}\right) \frac{\mathrm{d} \xi}{\mathrm{d} \xi}\right] \mathrm{d}=0 .
$$

Upon integration by parts, and in view of the arbitrariness of $\delta U_{1}(\xi)$ and $\delta U_{2}(\xi)$, one obtains two two-point boundary-value problems for $U_{1}(\xi)$ and $U_{2}(\xi)$, with $\xi$ in the interval $[0,1]$. The two systems are particularly simple [similar to (34) and (36), but with the boundary conditions set to zero]. Furthermore, the condition (29) of strong 
ellipticity in the overburden, which implies that the moduli $L_{1221}^{\prime}$ and $L_{2222}$ differ from zero, permits one to deduce from (49) that ${ }^{U}$ and $\stackrel{0}{U}_{2}$ are constants:

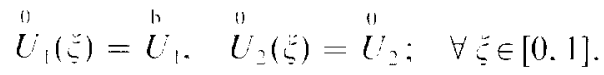

The asymplotic expansion of (46) is continued to the next term $\left(c^{\prime}\right)$ using the results (50):

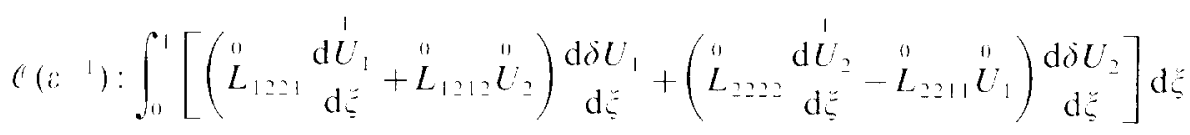

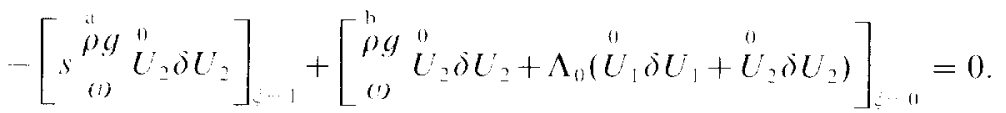

Here again. (51) yiclds two two-point boundary-value problems as soon as the arbitratiness of $\delta U_{1}(\zeta)$ and $\delta U_{2}(\zeta)$ is accounted for. The first problem, corresponding to $\delta L_{1}(\zeta)$ is

$$
\begin{aligned}
& -\underset{\mathrm{d} \zeta}{\mathrm{d}}\left[\begin{array}{cc}
0 & \mathrm{~d} U_{1}^{\prime}(\zeta) \\
L_{1221} & \mathrm{~d} \zeta
\end{array}\right. \\
& \ddot{L}_{1221} \mathrm{~d} \frac{U_{1}}{\mathrm{~d}}+\ddot{L}_{1212} \ddot{U}_{2}=0, \quad \zeta=1 ; \quad L_{1221} \mathrm{~d} U_{1}^{\prime}+\ddot{L}_{1212} \ddot{U}_{2}=\Lambda_{11} U_{1}, \quad \xi=0 .
\end{aligned}
$$

Given the fact that $\stackrel{0}{L}_{1221}=\ddot{L}_{1212}[$ see $(21)$ and (47)] and that both moduli differ from zero because of the condition of strong ellipticity (29), (52) simplifies to

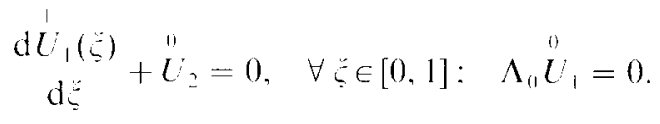

The second two-point boundary-value problem extracted from (51) and corresponding $10 \delta U_{2}(\zeta)$ reads

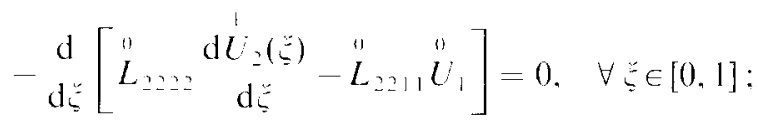

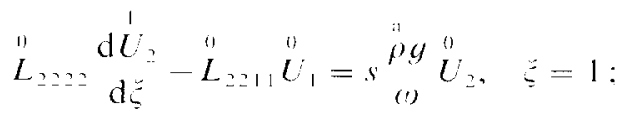

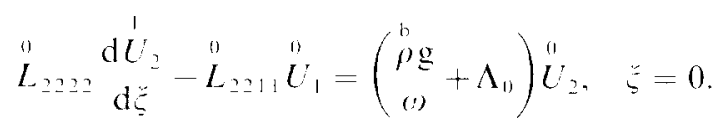

This is further simplified to 


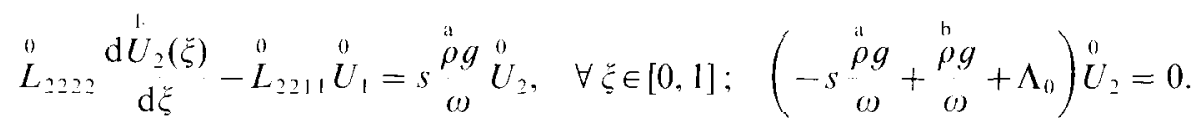

As before, stability depends on the sign of the largest possible real part of $\Lambda_{0}$ obtained from (53) and (55). For a substratum lighter than the overburden $(\stackrel{a}{\rho}>\stackrel{b}{\rho})$ two cases are distinguished depending on the erosion condition in a way that is analogous to the derivation of (38):

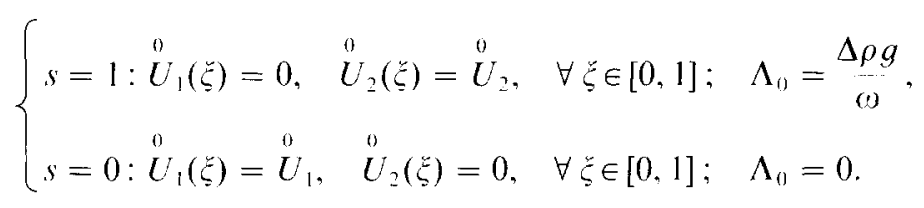

The physical interpretation of the above result is that the geological structure is always unstable with respect to long-wavelength perturbations, regardless of the prevailing in situ stress state, if the erosion condition is imposed on the top surface of the overburden $(s=1)$. In the absence of crosion $(s=0)$, higher-order terms in the expansion of $\Lambda$ are needed to determine the stability of the overburden. To this end, one has to consider the next order term in the expansion of (46) :

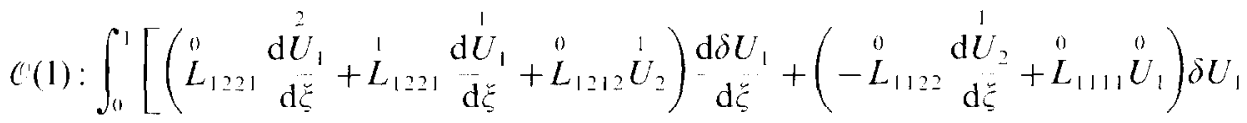

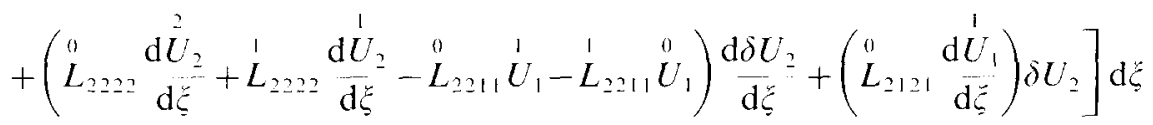

$$
\begin{aligned}
& +\left[\left(\frac{a}{\rho g} \frac{0}{\omega} U_{1}+\frac{\stackrel{\rho}{\rho g}}{\omega} U_{2}^{\prime}\right) \delta U_{2}+\Lambda_{1} \stackrel{o}{U}_{1} \delta U_{1}\right]_{\xi=0}=0
\end{aligned}
$$

As expected, two systems of differential equations and boundary conditions are associated with the terms $\delta U_{1}(\xi)$ and $\delta U_{2}(\xi)$. The system corresponding to $\delta U_{1}(\xi)$ is

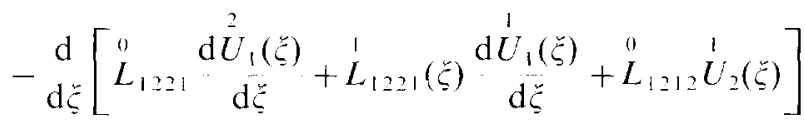

$$
\begin{aligned}
& +\left[\begin{array}{c}
0 \\
-L_{1122} \frac{\mathrm{d} U_{2}}{\mathrm{~d}} \dot{\xi}
\end{array}+\stackrel{0}{L_{1111}} \stackrel{0}{U}_{1}\right]=0, \quad \forall \xi \in[0,1], \\
& \text { i) } L_{1221} \frac{\stackrel{2}{U}^{\prime}}{\mathrm{d} \xi}+\stackrel{1}{L}_{1221} \frac{\mathrm{d} \dot{U}_{1}}{\mathrm{~d}}+\stackrel{0}{\xi}+L_{1212} \stackrel{1}{U}_{2}=0, \quad \xi=1 \text {; } \\
& \ddot{L}_{1221} \frac{\stackrel{2}{U}_{1}}{\mathrm{~d} \xi}+\stackrel{1}{L}_{1221} \frac{\mathrm{d} \dot{U}_{1}}{\mathrm{~d} \xi}+\stackrel{0}{L_{1212}} \stackrel{1}{U_{2}}=\Lambda_{1} \stackrel{0}{U}_{1}, \quad \xi=0 .
\end{aligned}
$$


A nontrivial solution for $U_{1}$, has to satisfy the compatibility condition discussed after (44). If such is the case, $(58)$ and $(55)_{1}$ imply that $\mathrm{d} U_{2} / \mathrm{d} \xi=\left(L_{22,1 /} / L_{2222}\right) \stackrel{0}{U_{1}}$ for $s=0$ and one obtains

$$
\Lambda_{1}=-\left(\begin{array}{ll}
0 & \frac{0}{L_{1122} L_{2211}} \\
L_{1111} & \frac{0}{L_{2222}}
\end{array}\right)
$$

This result has an interpretation similar to the one given for (45), which was obtained for a constant value of $\Delta \rho g H / G$. The right-hand side of (59) is the negative of the tangent modulus $E_{1} \equiv L_{1111}-L_{1122} L_{2211} / L_{2222}$ of the uniaxial force-displacement response. Hence. for a stress state corresponding to a displacement prior to the maximum load. $E_{1}$ is positive and the geological structure is stable with respect to long-wavelength perturbations. Conversely, negative values of $E_{1}$ imply instability. The absence of an integral on the right-hand side of (59), in contrast to (45), is due to the $\xi$-independence of $L_{i j k /}$. Notice also the absence of gravity effects in the present case of fixed perturbation wavelength $(\Delta \rho g / G(\omega)=$ const), as opposed to the case of fixed layer thickness $(\Delta \rho g H / G=$ const $)$.

3.1.3. Neutral stability curtes $(\Delta \rho g / G \omega=$ const $)$. The results obtained thus far determine the stability of points along the $\omega H=0$ axis in the $\omega H$ vs $\sigma_{0} / G$ stability diagrams. Given the interest in the boundary between the stable and unstable regions in these diagrams, i.e. the critical wavenumber $\left(\omega_{c}\right)$ curves for $\Delta \rho g H / G=$ const or the critical height $\left(H_{*}\right)$ curves for $\Delta \rho g / G \omega=$ const, attention is next focussed on obtaining these curves in the region of small $\omega H$ using the asymptotic technique developed above. The case $\Delta \rho g / G(1)=$ const is the easier of the two to investigate, since the asymptotic expression for the $H_{\mathrm{c}}$-curve can be obtained analytically.

The desired curve is found in implicit form from the requirement $\operatorname{Re}\left[\Lambda\left(\omega H, \sigma_{i /} / G, \Delta \rho g / G(\omega)\right]=0\right.$, where the stability exponent $\Lambda$ is calculated from (20). In all our numerical calculations, which are presented in the next section. the boundary between the stable and unstable regions in the $\omega H \mathrm{vs} \sigma_{0} / G$ stability diagrams always satisfies $\Lambda=0$, i.e. the critical point never corresponds to a flutter instability. Consequently, the critical layer thickness $H_{\mathrm{c}}$ can be found implicitly from

$$
\Lambda\left(c, \sigma_{0} / G, \Delta \rho g / G(\omega)=0\right.
$$

To avoid singular terms in the asymptotic expansions of $H_{c}$ as a function of $\sigma_{0 / G} G$ and $\Delta p g / G(\omega)$, one must fix $\varepsilon$ and $\sigma_{0 /} G$ and look for the $\theta-$ and $\sigma_{0 /} / G$-dependence of $\Delta \rho g / G \omega$ along the curve of neutral stability $\Lambda=0$. By expressing $(\Delta \rho g / G(j))_{c}=f^{\prime}\left(\sigma_{0 /} / G, i\right)$ in an asymptotic expansion with respect to $\varepsilon$, one obtains the desired critical layer thickness for any given value of the parameter $\Delta \rho g / G(\omega$.

The starting point for the asymptotic expansion of $(\Delta \rho g / G \omega)_{c}$ in terms of the small parameter $: \equiv \omega H$ along the neutral stability curve $(\Lambda=0)$ is once again $(20)$, where for convenience, the definitions $V_{1} \equiv U_{1} / x$ and $V_{2} \equiv U_{2}$ are now introduced: 


$$
\begin{aligned}
& \int_{0}^{1}\left[\left(L_{1221} \frac{\mathrm{d} V_{1}}{\mathrm{~d} \xi}+L_{1312} V_{2}\right) \frac{\mathrm{d} \delta V_{1}}{\mathrm{~d} \xi}+\left(-L_{1122} \frac{\mathrm{d} V_{2}}{\mathrm{~d} \xi}+\varepsilon^{2} L_{1111} V_{1}\right) \delta V_{1}\right. \\
& \left.+\left(\frac{1}{\varepsilon^{2}} L_{2222} \frac{\mathrm{d} V_{2}}{\mathrm{~d} \xi}-L_{2211} V_{1}\right) \frac{\mathrm{d} \delta V_{2}}{\mathrm{~d} \xi}+\left(L_{2121} \frac{\mathrm{d} V_{1}}{\mathrm{~d} \xi}+L_{2112} V_{2}\right) \delta V_{2}\right] \mathrm{d} \ddot{\xi} \\
& -\left[\frac{1}{\delta} s \frac{\mathrm{a}}{\rho} \frac{G}{\Delta \rho}\left(\frac{\Delta \rho g}{G \omega}\right)_{c} V_{2} \delta V_{2}\right]_{\xi=1}+\left[\varepsilon \frac{\rho G}{\Delta \rho}\left(\frac{\Delta \rho g}{G \omega}\right)_{s}\left(V_{1} \delta V_{2}+V_{2} \delta V_{1}\right)\right. \\
& \left.+\frac{1}{\varepsilon} \frac{\partial G}{\Delta \rho}\left(\frac{\Delta \rho g}{G \omega}\right) V_{2} \delta V_{2}\right]_{\xi=0}=0
\end{aligned}
$$

By inspection, the adopted asymptotic expansions for $V_{i}$ and $(\Delta \rho g / G \omega)_{c}$ in terms of $c$ are

$$
\begin{aligned}
& V_{i}(\xi, \varepsilon)=\stackrel{0}{V}_{i}(\xi)+\varepsilon^{2} V_{i}(\xi)+\varepsilon^{4} \dot{V}_{i}(\xi)+\mathcal{O}\left(\varepsilon^{6}\right), \\
& \left(\frac{\Delta \rho g}{G \omega}\right)_{c}=\varepsilon\left[\gamma_{0}+\varepsilon^{2} \gamma_{2}+\varepsilon^{4} \gamma_{4}+O\left(s^{6}\right)\right] .
\end{aligned}
$$

It follows from (27) and (62) that the overburden stresses have the following dependence on $\varepsilon$ :

$$
\begin{aligned}
& \sigma_{13}=\sigma_{0} \cos (\varphi)+\varepsilon^{2} k_{1} \frac{\rho G}{\Delta \rho} y_{0} \zeta+\sigma\left(\varepsilon^{4}\right) \\
& \sigma_{22}=\varepsilon^{2} \frac{\rho G}{\Delta \rho} \gamma_{0}(\xi-1)+O\left(a^{4}\right) \\
& \sigma_{33}=\sigma_{0} \sin (\varphi)+\varepsilon^{2} k_{3} \frac{\stackrel{\mu}{\rho} G}{\Delta \rho} \gamma_{0} \xi+Q\left(\varepsilon^{4}\right) .
\end{aligned}
$$

In view of (21)-(26), the equations of (63) imply the following asymptotic expansion for the incremental moduli:

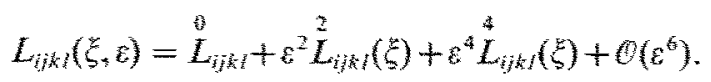

Notice that the -independence of the 0 (1) term in the stresses implies a similar independence of the $O(1)$ term in the expansion of the incremental moduli $L_{i j / k}$. The mode normalization condition is given once again by (32), which in view of the new parametrization of the eigenmode $V$, introduced in (61), becomes $\int_{0}^{1}\left[V_{2} V_{2}+\varepsilon^{2} V_{1} V_{1}\right] d \xi=1$. For simplicity, the slightly modified normalization condition $\int_{0}^{1} V_{2} V_{2} \mathrm{~d} \xi=1$ will be employed in the rest of this subsection.

If one substitutes the asymptotic expressions (62) and (64) into (61), expands the results in terms of ascending powers of $\varepsilon$ and then groups the terms of the same order in $\varepsilon$, one gets the following results : for the $O\left(\varepsilon^{-2}\right)$ term of $(61)$ one obtains (49) once 
more (with $\ddot{U}_{1}=0$ and $\ddot{U}_{2}=\ddot{V}_{2}$, which have been shown to imply that ${ }^{\prime}$ is constant). The modified normalization condition yields $\int_{01}^{1} V_{2} V_{2}=1$ which implies that $V_{2}=1$. The next term of $c(1)$ in (61) gives, after also taking into account that $\mathrm{d}_{2}^{0} \mathrm{~d} \xi=0$,

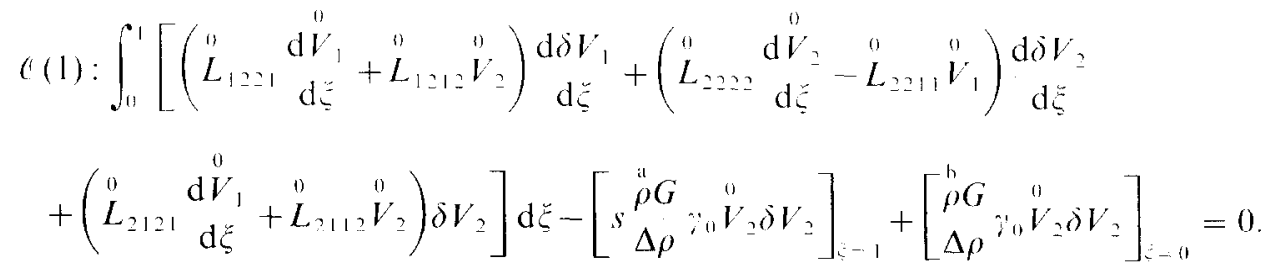

Upon integration of (65) by parts, and in view of the arbitrariness of $\delta V_{1}(\xi)$ and $\delta V_{2}(\xi)$, one obtains two two-point boundary-value problems. The system corresponding to $\delta V_{1}(\zeta)$ is

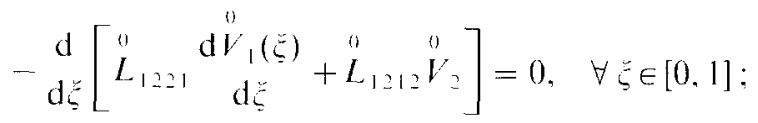

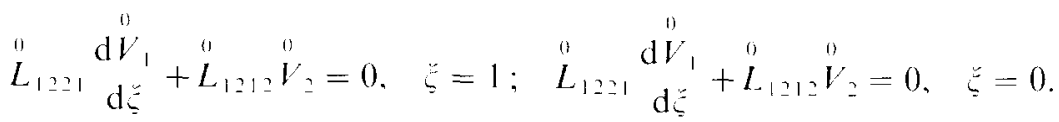

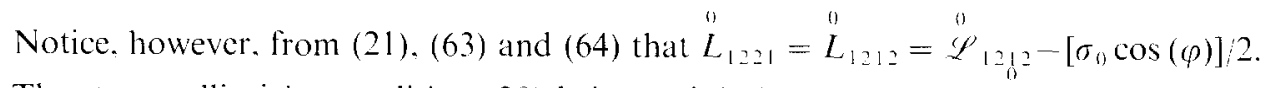
The strong ellipticity condition (29) being satisfied, we know that $L_{1212}$ differs from zero and rewrite (66) in the simplified manner : $\stackrel{0}{L}_{1212}\left(\mathrm{~d} V_{1} / \mathrm{d} \xi+\stackrel{0}{V_{2}}\right)=0$ for $\xi \in[0,1]$. The simplified expression for (66) and its solution read

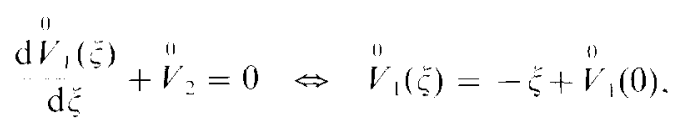

in which $\stackrel{9}{V}_{2}=1$ has been used. The second problem obtained from $(65)$ and corresponding to $\delta V_{2}(\xi)$ is

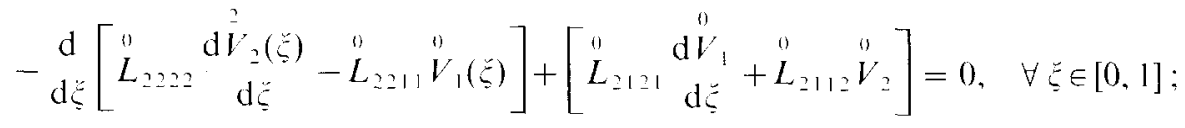

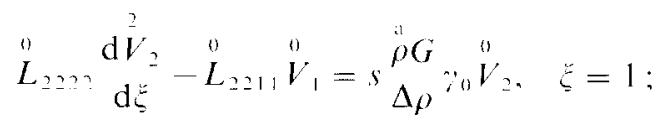

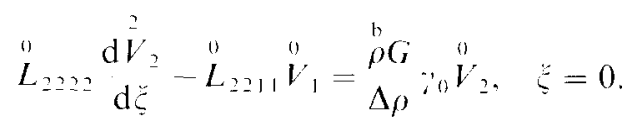


The above system has a form similar to $(44):-\mathrm{d} f / \mathrm{d} \xi+g=0$ for $\xi \in[0,1]$ with $f(0)=a$ and $f(1)=b$. Similarly, to permit a solution, the function $g(\xi)$ and the scalars $a$ and $b$ also have to satisfy the compatibility condition $\int_{0}^{1} g(\xi) \mathrm{d} \xi=b-a$. Since the definition of the moduli (21) and the asymptotic development (63) and (64) indicate that $\stackrel{0}{L}_{2112}-\stackrel{0}{L}_{2121}=\sigma_{0} \cos (\varphi)$, one obtains from the compatibility condition and (67)

$$
\begin{aligned}
& \int_{0}^{1}\left(\stackrel{0}{L} L_{2112}-\stackrel{0}{L}_{2121}\right) \stackrel{0}{V_{2}} \mathrm{~d} \xi=\sigma_{0} \cos (\varphi) \stackrel{0}{V_{2}}=(\stackrel{\mathrm{a}}{\rho} \rho-\stackrel{\mathrm{b}}{\rho}) \frac{G}{\Delta \rho} \gamma_{0} \stackrel{0}{V}_{2} \\
& \Leftrightarrow \quad \gamma_{0}=\underset{s \rho-\rho}{\Delta \rho}\left(\frac{\sigma_{0}}{G}\right) \cos (\varphi) .
\end{aligned}
$$

Consequently, from (67)-(69), the fact that $V_{2}=1$ and the modified normalization condition $\int_{0}^{1} V_{2} V_{2} \mathrm{~d} \xi=1$, which implies that $\int_{0}^{1} \stackrel{V}{V}_{2} \underline{V}_{2}^{2}=0$, yield the following result for $\bar{V}_{2}(\xi)$ :

$$
\begin{aligned}
\vec{V}_{2}(\xi)=\frac{1}{0}-\left\{\frac { 0 } { L _ { 2 2 2 2 } } \left[\left(-\frac{\xi^{2}}{2}+\frac{1}{6}\right)\right.\right. & \left.+\left(\xi-\frac{1}{2}\right) V_{1}^{0}(0)\right] \\
& \left.+\left[\left(\frac{\xi^{2}}{2}-\frac{1}{6}\right)(s \rho-\rho)+\left(\xi-\frac{1}{2}\right) \stackrel{\mathrm{b}}{\rho}\right] \frac{G}{\Delta \rho} \gamma_{0}\right\} .
\end{aligned}
$$

The next term in the expansion of $(61)$ is

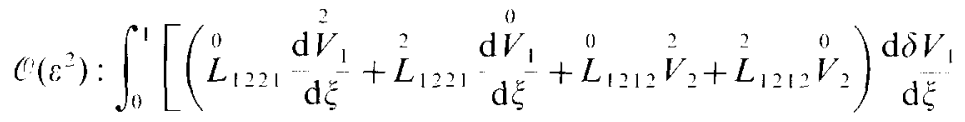

$$
\begin{aligned}
& +\left(-\stackrel{0}{L_{1122}} \frac{\mathrm{d} \stackrel{2}{V}_{2}^{2}}{\mathrm{~d} \xi^{2}}+\stackrel{0}{L_{1111}} \stackrel{0}{V_{1}}\right) \delta V_{1} \\
& +\left(\stackrel{0}{L}_{2222} \frac{\stackrel{4}{V}_{2}}{\mathrm{~d} \xi}+\stackrel{2}{L_{2222}} \frac{\mathrm{d} \stackrel{2}{V}_{2}}{\mathrm{~d} \xi}-\stackrel{0}{L}_{2211} \stackrel{2}{V}_{1}-\stackrel{2}{L}_{2211} \stackrel{0}{V}_{1}\right) \frac{\mathrm{d} \delta V_{2}}{\mathrm{~d} \xi} \\
& \left.+\left(\stackrel{0}{L_{2121}} \frac{\mathrm{d} \dot{V}_{1}}{\mathrm{~d} \xi}+\stackrel{2}{\xi_{2121}} \frac{\mathrm{d} V_{1}}{\mathrm{~d} \xi}+\stackrel{0}{L}_{2112} \stackrel{2}{V}_{2}+\stackrel{2}{L}_{2112} \stackrel{0}{V_{2}}\right) \delta V_{2}\right] \mathrm{d} \xi \\
& -\left[\stackrel{a}{\rho} \frac{a}{\Delta \rho}\left(\gamma_{2} \stackrel{0}{V}_{2}+\gamma_{0} V_{2}^{2}\right) \delta V_{2}\right]_{\xi=1} \\
& +\left[\stackrel{a}{\rho} \frac{G}{\Delta \rho} \gamma_{0}\left(V_{1} \delta V_{2}+\stackrel{0}{V_{2}} \delta V_{1}\right)+\frac{\stackrel{b}{\rho} G}{\Delta \rho}\left(\gamma_{2} \stackrel{0}{V_{2}}+\gamma_{0} \stackrel{2}{V}\right) \delta V_{2}\right]_{\xi=0}=0,
\end{aligned}
$$


in which the condition $\mathrm{d} \stackrel{0}{V}_{2} / \mathrm{d} \xi=0$ has been exploited. Treating (71) like (65), one obtains from (71) two systems of differential equations plus boundary conditions. The system corresponding to $\delta V_{1}(\xi)$ is

$$
\begin{aligned}
& -\frac{\mathrm{d}}{\mathrm{d} \xi}\left[\stackrel{0}{L}_{1221} \frac{\mathrm{d} \vec{V}_{1}(\xi)}{\mathrm{d} \xi}+\stackrel{2}{L}_{1221}(\xi) \frac{\mathrm{d}^{V}}{\mathrm{~d} \xi}+\stackrel{0}{L}_{1212} \stackrel{2}{V}_{2}(\xi)+\stackrel{2}{L}_{1212}(\xi) \stackrel{0}{V_{2}}\right] \\
& +\left[-\stackrel{0}{L}_{1122} \frac{\mathrm{d} \stackrel{2}{V}_{2}(\xi)}{\mathrm{d} \xi}+\stackrel{0}{L_{1111}} \stackrel{0}{V}_{1}(\xi)\right]=0, \quad \forall \xi \in[0,1] \\
& \stackrel{0}{L}_{12 ? 1} \frac{\mathrm{d} \dot{V}_{1}}{\mathrm{~d} \xi}+\stackrel{2}{L_{1221}} \frac{\mathrm{d} V_{1}}{\mathrm{~d} \xi}+\stackrel{0}{L_{1212}} \stackrel{2}{V}_{2}+\stackrel{2}{L}_{1212} \stackrel{0}{V_{2}}=0, \quad \xi=1,
\end{aligned}
$$

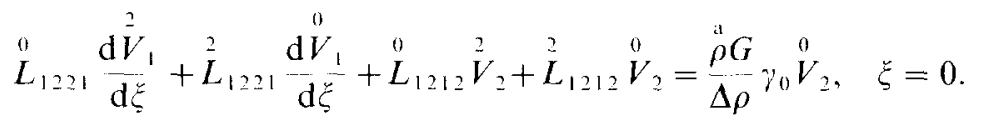

Note that the above system has a structure similar to (44) and (68). We thus conclude that a solution is possible if the following compatibility condition is satisfied:

$$
\int_{0}^{1}\left[-\stackrel{0}{L}_{1122} \frac{\mathrm{d} V_{2}^{2}(\xi)}{\mathrm{d} \xi}+\stackrel{0}{L}_{1111} V_{1}(\xi)\right] \mathrm{d} \xi=-\frac{\stackrel{\rho}{\rho} G}{\Delta \rho} \gamma_{0} V_{2}
$$

If such a condition is fulfilled, and bearing in mind that $\stackrel{0}{V}_{2}=1$, the substitution of (67) and (70) into (73) yields

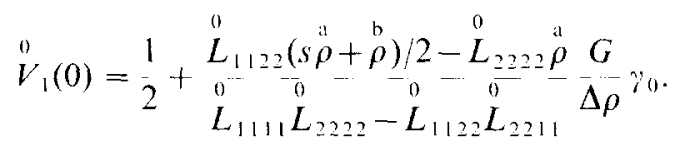

Consequently, one deduces from $(67),(70)$ and (72) that

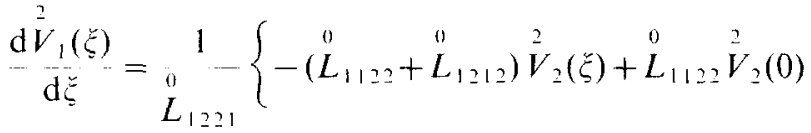

$$
\begin{aligned}
& \left.+\ddot{L}_{1111}\left[-\frac{\xi^{2}}{2}+\stackrel{0}{V_{1}}(0)\right]+\xi \frac{\stackrel{\rho}{\rho} G}{\Delta \rho} \gamma_{0}\right\} .
\end{aligned}
$$

The derivation of (75) also required the result obtained from (21), (63) and (64) namely the relation $\stackrel{2}{L}_{1221}(\xi)-\stackrel{2}{L}_{1212}(\xi)=\left(\stackrel{a}{\rho} G / \Delta \rho \gamma_{2}\right) \gamma_{0}(\xi-1)$. Note also that the righthand side of $(75)$ is completely specified, since $\tilde{V}_{2}(\xi), V_{1}(0)$ and $\gamma_{0}$ are known from $(70),(74)$ and (69), respectively. The second system obtained from (71) and corresponding to $\delta V_{2}(\xi)$ is 


$$
\begin{aligned}
& -\frac{\mathrm{d}}{\mathrm{d} \xi}\left[\stackrel{0}{L_{2222}} \frac{\mathrm{d} V_{2}(\xi)}{\mathrm{d} \xi}+\stackrel{2}{L}_{2222}(\xi) \frac{\mathrm{d} \stackrel{2}{V}_{2}(\xi)}{\mathrm{d} \xi}-\stackrel{0}{L}_{2211} \stackrel{2}{V}_{1}(\xi)-\stackrel{2}{L}_{2211}(\xi) \stackrel{0}{V},(\xi)\right] \\
& +\left[\stackrel{0}{L}_{2121} \frac{\mathrm{d}^{2} V_{1}(\xi)}{\mathrm{d} \xi}+\stackrel{2}{L}_{2121}(\xi) \frac{\mathrm{d} V_{1}}{\mathrm{~d} \xi}+\stackrel{0}{L}_{2112} \stackrel{2}{V}_{2}(\xi)+\stackrel{2}{L}_{2112}(\xi) \stackrel{0}{V_{2}}\right]=0, \quad \forall \xi \in[0,1], \\
& \stackrel{0}{L}_{2222} \frac{\mathrm{d}^{4}}{\mathrm{~d}} \underset{2}{\xi}+\stackrel{2}{L_{2222}} \frac{\mathrm{d} \stackrel{2}{V}_{2}}{\mathrm{~d} \xi}-\stackrel{0}{L_{2211}} \stackrel{2}{V}_{1}-\stackrel{2}{L}_{2211} \stackrel{n}{V_{1}}=s \frac{\stackrel{\mathrm{a}}{\rho} G}{\Delta \rho}\left(\gamma_{2} \stackrel{0}{V}_{2}+\gamma_{0} \stackrel{2}{V}_{2}\right), \quad \xi=1, \\
& \stackrel{0}{L_{2222}} \frac{\mathrm{d} V_{2}^{2}}{\mathrm{~d} \xi}+\stackrel{2}{L}_{2222} \frac{\mathrm{d} \stackrel{2}{V}_{2}}{\mathrm{~d} \xi}-\stackrel{0}{L}_{2211} \stackrel{2}{V}_{1}-\stackrel{2}{L_{2211}} \stackrel{0}{V}_{1}=\frac{\stackrel{\mathrm{a}}{\rho} G}{\Delta \rho} \gamma_{0} \stackrel{0}{V}_{1}+\frac{\stackrel{\mathrm{h}}{\rho} G}{\Delta \rho}\left(\vartheta_{2} \stackrel{0}{V}_{2}+\gamma_{0} \stackrel{2}{V}_{2}\right), \quad \xi=0 .
\end{aligned}
$$

Working with (76) in the same way as with (44), (68) and (72), one finds that a solution to (76) is possible if the following compatibility condition is satisfied:

$$
\begin{aligned}
& \int_{0}^{1}\left[\stackrel{0}{L}_{2121} \frac{\mathrm{d} \stackrel{2}{V}_{1}(\xi)}{\mathrm{d} \xi}+\stackrel{2}{L}_{2121}(\xi)\right.\left.\frac{\mathrm{d} V_{1}}{\mathrm{~d} \xi}+\stackrel{0}{L}_{2112} \stackrel{2}{V}_{2}(\xi)+\stackrel{2}{L}_{2112}(\xi) \stackrel{0}{V}_{2}\right] \mathrm{d} \xi= \\
&(s \stackrel{\mathrm{a}}{\rho}-\stackrel{\mathrm{b}}{\rho}) \frac{G}{\Delta \rho} \gamma_{2}+\left[\mathrm{s}^{\mathrm{a}} \stackrel{2}{V}_{2}(1)-\stackrel{\mathrm{b}}{\rho} \stackrel{2}{V}_{2}(0)-\stackrel{\stackrel{a}{\rho}^{V}}{V_{1}}(0)\right] \frac{G}{\Delta \rho} \gamma_{0} .
\end{aligned}
$$

From (21), (63) and (64) the following equalities are established:

$$
\int_{0}^{1}\left[L_{2112}(\xi)-\stackrel{2}{L}_{2121}(\xi)\right] \mathrm{d} \xi=k_{1}(\stackrel{a}{\rho} G / \Delta \rho)\left(\gamma_{0} / 2\right) \text { and } \stackrel{0}{L}_{2121}=\ddot{L}_{1221}
$$

The integral of $\left(\mathrm{d}^{2} V_{1} / \mathrm{d} \xi\right)$ that appears in (77) is calculated once (75) is substituted into it. Note also the simplification: $\int_{0}^{1} \stackrel{2}{V}_{2} \mathrm{~d} \xi=0$, from (70). With the help of (67), (70) and (74), the following solution for $\gamma_{2}$ is obtained from (77):

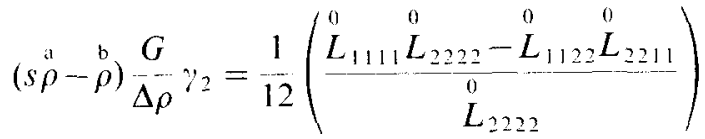

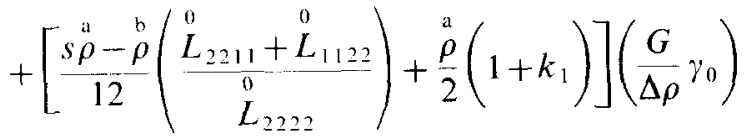

$$
\begin{aligned}
& -\frac{1}{L_{2222}}\left\{\frac{\left[L_{1122}(s \stackrel{\mathrm{a}}{\rho}+\stackrel{\mathrm{b}}{\rho}) / 2-\stackrel{0}{L}_{2222} \stackrel{\mathrm{a}}{\rho}\right]\left[\stackrel{0}{L_{2211}}(\stackrel{\mathrm{a}}{\rho} \rho+\stackrel{\mathrm{b}}{\rho}) / 2-\stackrel{0}{L}_{2222} \stackrel{a}{\rho}\right]}{0}\right. \\
& \left.+\frac{1}{3}\left[(s \stackrel{\mathrm{a}}{\rho})^{2}+s \stackrel{\mathrm{a} \rho}{\rho} \rho+(\stackrel{\mathrm{b}}{\rho})^{2}\right]\right\}\left(\frac{G}{\Delta \rho} \gamma_{0}\right)^{2} \text {. }
\end{aligned}
$$

Finally, from (62), (67) and (78) we find that the following relation between 
$\left(\Delta \rho g G(\omega), \omega H\right.$ and $\sigma_{0} G$ holds at neutral stability $(\Lambda=0)$ for small values of the parameter $a \equiv(1) H\left[\right.$ up to $\left.\left(c^{5}\right)\right]$ :

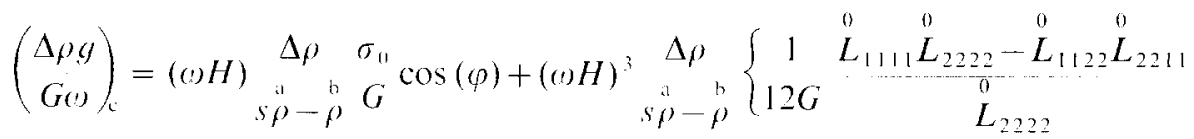

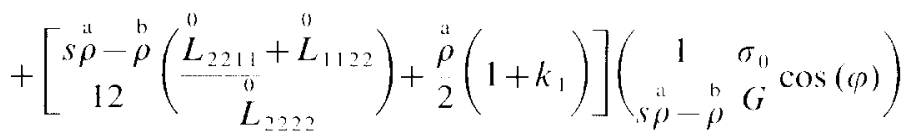

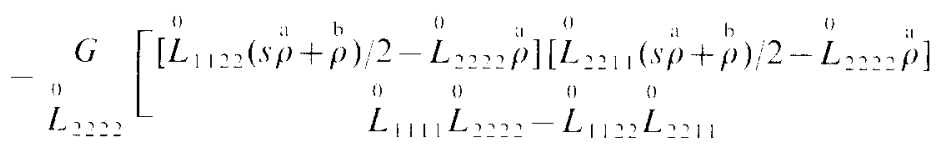

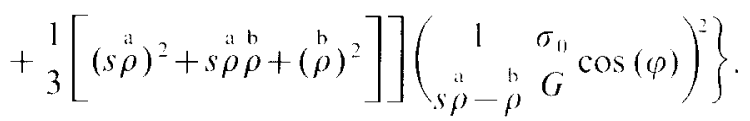

Note that. in view of (21), (27) and (63). (64), the normalized moduli $\stackrel{0}{L}, i k /^{\prime} G$ are functions of the normalized stress $\sigma_{0} / G$. As a result, the desired relation between $\sigma_{0} G$ and $(H$ at neutral stability $(\Lambda=0)$, which is implicit, can be solved for a given $\Delta \rho, G(:)$ by any convenient means.

The above result is considerably simplified if the lop layer responds elastically, since

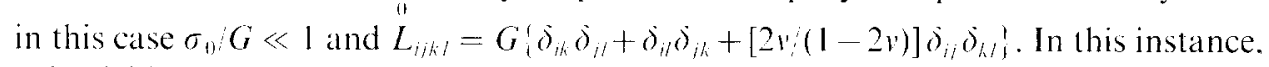
(79) yiclds

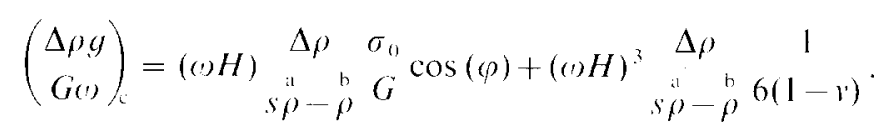

At neutral stability, that equation tells us that the critical stress, $\left(\sigma_{0}\right)_{\text {, }}$, for the onset of buckling of an elastic beam resting atop a fluid bed is

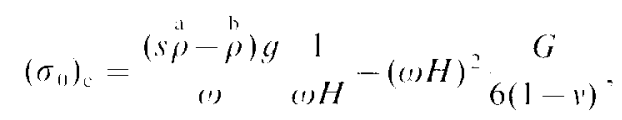

if we set the stress orientation angle $\varphi$ to zero. In the absence of erosion $(s=0)$. we find that ( 80 ) is equivalent to Equation (9) of the paper by RAMBERG and STEPHANSSON (1964). Interestingly enough, these authors traced the original publication of this result to the beginning of the century [SMOLUCHOwSKI (1909); see RAMBERG and STEPHANSSON (1964) for further reference].

The derivation of (80) lor the particular case of an clastic overburden, holds for perturbations with small wave number. The equation is thus valid only near the origin of the stability diagrams. Nevertheless, it will prove useful in the discussion of Section 4 , since the neutral stability curves in the compressive range often lie in that region of the stability diagrams. For that reason, and in view of the simplicity of $(80)$ compared to (79), a brief discussion of the case of an overburden with an elastic response is appropriate. In the absence of erosion $(s=0)$, the two terms present on the right-hand side of (80) are negative and have a dominant contribution for either 
large or small values of $\omega H$. There thus exists an absolute-value minimum compressive stress $\sigma_{m}$, which is given by

$$
\sigma_{\mathrm{m}}(\omega)=-\frac{3}{2}\left[\left(\frac{\rho g}{\omega}\right)^{2} \frac{G}{3(1-v)}\right]^{1 / 3} .
$$

If $\sigma_{0}>\sigma_{\mathrm{m}}$, we should expect stability in the compressive range of deformation for any adequately small $\omega H$ in view of the stability of the system to perturbations of infinitely long wavelength, provided the redistribution condition does not apply [see discussion after (59)]. Since this result was obtained for a fixed wave number $(\%$, overburdens compressed by a stress larger than $\sigma_{\mathrm{m}}$ are stable regardless of their thick ness.

The introduction of erosion changes this picture completely. For $s=1$, the two contributions on the right-hand side of (80) are of a different sign. The stress $\left(\sigma_{0}\right)_{c}$ changes sign for some critical value of $\omega H$. There therefore exists no maximum in the compressive stress, in contrast to the case in which there is no redistribution at the top surface. The stress changes sign for the critical height:

$$
H_{0}=\left(\begin{array}{c}
6(1-v)(\stackrel{a}{\rho}-\stackrel{b}{\rho}) g \\
G \omega^{4}
\end{array}\right)^{1.3} .
$$

We have already learned [see discussion following (56)] that in the presence of erosion, the system is unstable with respect to perturbations having a vanishing wave number. The minimum layer thickness for stability under no lateral stress $\left(\sigma_{0}=0\right)$ is provided by $(81)$. Comparing the results obtained here for a system at neutral stability with or without erosion, we conclude that the redistribution condition is destabilizing and has a predominant effect on shallow overburdens.

\subsection{Short-watelength limit $(\omega H \rightarrow \infty)$}

We now turn our attention to the short-wavelength limit for constant values of $\Delta \rho g H / G$ and $\Delta \rho g / \omega G$. In the latter case, we establish a link with the analysis of TRIANTAFYLLIDIS and LEHNER (1993), which was concerned with the interfacial instability between two half-spaces.

3.2.1. Fixed layer thickness $(\Delta \rho g H / G=\mathrm{const}$ ). The starting point for our study of stability with respect to disturbances whose wavelength is much shorter than the height of the overburden is again the variational formulation (20). For reasons that will become apparent below, the corresponding Euler-Lagrange equations and boundary conditions are needed:

$$
\left.\begin{array}{r}
-\varepsilon \frac{\mathrm{d}}{\mathrm{d} \xi}\left[\varepsilon L_{1221} \frac{\mathrm{d} U_{1}}{\mathrm{~d} \xi}+L_{1212} U_{2}\right]+\left[-\varepsilon L_{1122} \frac{\mathrm{d} U_{2}}{\mathrm{~d} \xi}+L_{1111} U_{1}\right]=0 \\
-\varepsilon \frac{\mathrm{d}}{\mathrm{d} \xi}\left[\varepsilon L_{2222} \frac{\mathrm{d} U_{2}}{\mathrm{~d} \xi}-L_{2211} U_{1}\right]+\left[\varepsilon L_{2121} \frac{\mathrm{d} U_{1}}{\mathrm{~d} \xi}+L_{2112} U_{2}\right]=0
\end{array}\right\} \forall \xi \in[0,1] ;
$$




$$
\begin{aligned}
& \left.\begin{array}{c}
\varepsilon L_{1221} \frac{\mathrm{d} U_{1}}{\mathrm{~d} \xi}+L_{1212} U_{2}=0 \\
\varepsilon L_{2222} \frac{\mathrm{d} U_{2}}{\mathrm{~d} \xi}-L_{2211} U_{1}=\operatorname{ss\rho g} \rho U_{2}
\end{array}\right\} \xi=1 ; \\
& \left.\begin{array}{l}
\delta L_{1221} \frac{\mathrm{d} U_{1}}{\mathrm{~d} \xi}+L_{1212} U_{2}=\stackrel{a}{\rho} g H U_{2}+\Lambda U_{1} \\
\varepsilon L_{2222} \frac{\mathrm{d} U_{2}}{\mathrm{~d} \xi}-L_{2211} U_{1}=\stackrel{a}{\rho} g H U_{1}+(\rho \rho \rho g H+\Lambda) U_{2}
\end{array}\right\} \xi=0 .
\end{aligned}
$$

A new definition for the small parameter, $\varepsilon \equiv 1 / \omega H$, has been introduced here. Notice from (82)-(84) that the highest-order derivatives are multiplied by the small parameter $(\varepsilon \ll 1)$ which suggests the existence of a boundary layer in the system's solution. The eigenmode vector $U,(\xi, n)$ is expected to decay exponentially away from the interface $(\xi=0)$. since, according to $(84)$, these are the boundary conditions that involve the desired stability exponent $\Lambda$. A standard WKB asymptotic expansion [see, for example, Bender and Orszag (1978)] is the analytical tool of use in this case. According to such an expansion,

$$
\begin{aligned}
U_{i}(\xi, \varepsilon) & =\sum_{\alpha=1}^{2} U_{i}^{\alpha}(\xi, \varepsilon) \exp \left[\frac{1}{\varepsilon}\left(f_{\alpha}(\xi)+\varepsilon f_{x}(\xi)+\varepsilon^{2} f_{\alpha}(\xi)+c\left(\varepsilon^{3}\right)\right)\right] ; \\
U_{i}^{\alpha}(\xi, \varepsilon) & =U_{i}^{0}(\xi)+\varepsilon U_{i}^{\alpha}(\xi)+\varepsilon \dot{U}_{i}^{\alpha}(\xi)+c\left(\varepsilon^{3}\right) \\
\Lambda & =\Lambda_{0}+\varepsilon \Lambda_{1}+\varepsilon^{2} \Lambda_{2}+c\left(\varepsilon^{3}\right) .
\end{aligned}
$$

The boundary condition $f_{x}(0)=0, \forall j \in \mathbb{N}$ should be added to (85) for completeness. At this point, one should also recall the remark made in Subsection 3.1.1 according to which, for a fixed layer thickness $(\Delta \rho g H / G=$ const $)$, the incremental moduli in (82)-(84) depend solely on $\xi: L_{i / k}=L_{i j k /}(\xi)$.

Substitution of (85) into (82) gives for the lowest order in $\varepsilon$, which is the $c(1)$ term, two sets of equations, each consisting of two equations for the two unknowns $\ddot{U}_{i}^{\chi}(\zeta),(x=1,2 ; i=1,2)$. The resulting system evaluated on the interface $(\xi=0)$ is

$$
\begin{aligned}
& {\left[-L_{1221}(0)\left(\begin{array}{l}
\mathrm{d} f_{\gamma} \\
\mathrm{d} \xi
\end{array}\right)^{2}+L_{1111}(0)\right] \stackrel{0}{U}_{i}^{\alpha}(0)-\left[\left(L_{1122}(0)+L_{1212}(0)\right) \frac{\mathrm{d} f_{\alpha}}{\mathrm{d} \xi}\right] \stackrel{0}{U}_{2}^{\alpha}(0)=0,} \\
& {\left[\left(L_{2211}(0)+L_{2121}(0)\right) \frac{\mathrm{d} f_{\alpha}}{\mathrm{d} \xi}\right] \stackrel{0}{U_{1}^{\alpha}}(0)+\left[-L_{2222}(0)\left(\frac{\mathrm{d} f_{\mathrm{x}}}{\mathrm{d} \xi}\right)^{2}+L_{2112}(0)\right] \stackrel{U}{U}_{2}^{\alpha}(0)=0 .}
\end{aligned}
$$

The corresponding $C(1)$ term resulting from the substitution of $(85)$ into the boundary condition (84) at the interface $(\xi=0)$ is 


$$
\begin{gathered}
\sum_{\alpha=1}^{2}\left[\left(L_{1221}(0) \frac{\mathrm{d} f_{\alpha}}{\mathrm{d} \xi}-\Lambda_{0}\right) \stackrel{0}{U_{1}^{\alpha}}(0)+\left(L_{1212}(0)-\stackrel{\mathrm{a}}{\rho} g H\right) \stackrel{0}{U}_{2}^{\alpha}(0)\right]=0 \\
\sum_{\alpha=1}^{2}\left[-\left(L_{2211}(0)+\stackrel{\mathrm{a}}{\rho} g H\right) \stackrel{0}{U}_{1}^{\alpha}(0)+\left(L_{2222}(0) \frac{\mathrm{d} \tilde{f}_{\alpha}}{\mathrm{d} \xi}-\Lambda_{0}\right) \stackrel{0}{U_{2}^{\alpha}}(0)\right]=0
\end{gathered}
$$

The leading term $\Lambda_{0}$ in the expansion of the stability exponent is found from (86) and (87) as follows. Equation (86) yields the following relation between $U_{1}^{x}(0)$ and $\stackrel{0}{U^{x}}(0)$ :

$$
\stackrel{0}{U_{2}^{\alpha}}(0)=\gamma_{\alpha}^{0} U_{1}^{\alpha}(0) ; \quad \gamma_{\alpha} \equiv \frac{-L_{1221}(0)\left(z_{\alpha}\right)^{2}+L_{1111}(0)}{\left(L_{1122}(0)+L_{1212}(0)\right) z_{\alpha}}, \quad \alpha=1,2,
$$

where the $z_{x}$, defined as $\left(\mathrm{d} f_{x} / \mathrm{d} \xi\right)(0)$, are the two roots with negative real part $\left[\operatorname{Re}\left(z_{x}\right)<0\right]$ of

$$
\begin{aligned}
& {\left[L_{2222}(0) z_{\alpha}^{2}-L_{2112}(0)\right]\left[L_{1221}(0) z_{\alpha}^{2}-L_{1111}(0)\right]} \\
& +\left[L_{1122}(0)+L_{1212}(0)\right]\left[L_{2211}(0)+L_{2121}(0)\right] z_{\alpha}^{2}=0 .
\end{aligned}
$$

The existence of roots of (89) with nonzero real part is guaranteed by the requirement that all points in the geological structure be in the elliptic regime [i.e. no shear bands are permitted, see (29)]. The requirement that $\operatorname{Re}\left(z_{x}\right)<0$ follows from (85) and ensures the decay of the eigenmode away from the interface for $\xi>0$. Notice from (88) and (85) that

$$
\begin{gathered}
\sum_{x=1}^{2} \stackrel{0}{U}_{1}^{x}(0)=U_{1}(0,0) ; \\
\sum_{x=1}^{2} \stackrel{0}{U}_{2}^{x}(0)=U_{2}(0,0) ; \\
\sum_{x=1}^{2} \frac{\mathrm{d} f_{x}}{\mathrm{~d} \xi}(0) \stackrel{0}{U_{1}^{\alpha}}(0)=\frac{z_{1} \gamma_{2}-z_{2} \gamma_{1}}{\gamma_{2}-\gamma_{1}} U_{1}(0,0)+\frac{z_{2}-z_{1}}{\gamma_{2}-\gamma_{1}} U_{2}(0,0) ; \\
\sum_{x=1}^{2} \frac{\mathrm{d} f_{x}}{\mathrm{~d} \xi}(0) \stackrel{0}{U}_{2}^{x}(0)=-\gamma_{1} \gamma_{2} \frac{z_{2}-z_{1}}{\gamma_{2}-\gamma_{1}} U_{1}(0,0)+\frac{z_{2} \gamma_{2}-z_{1} \gamma_{1}}{\gamma_{2}-\gamma_{1}} U_{2}(0,0) .
\end{gathered}
$$

Upon substitution of these results into (87), the following $2 \times 2$ system for $U_{1}(0,0)$ and $U_{2}(0,0)$ is obtained :

$$
\begin{gathered}
\sum_{\beta=1}^{2}\left(S_{\alpha \beta}-\Lambda_{0} \delta_{\alpha \beta}\right) U_{\beta}(0,0)=0 \\
S_{11} \equiv L_{1221}(0) \frac{z_{1} \gamma_{2}-z_{2} \gamma_{1}}{\gamma_{2}-\gamma_{1}}, \quad S_{12} \equiv L_{1221}(0) \frac{z_{2}-z_{1}}{\gamma_{2}-\gamma_{1}}+L_{1212}(0)-\stackrel{\mathrm{a}}{\rho} g H \\
S_{21} \equiv-L_{2222}(0) \frac{z_{2}-z_{1}}{\gamma_{2}-\gamma_{1}} \gamma_{1} \gamma_{2}-L_{2211}(0)-\stackrel{a}{\rho} g H, \quad S_{22} \equiv L_{2222}(0) \frac{z_{2} \gamma_{2}-z_{1} \gamma_{1}}{\gamma_{2}-\gamma_{1}}
\end{gathered}
$$


Equations (91) show that $\Lambda_{0}$ is the eigenvalue of the matrix $S_{2 \beta}$ whose components are found from $L_{i \not k}(0)$. i.e. the values of the incremental moduli at the interface given by (88) and (89). Notice that the stability with respect to short-wavelength interface disturbances. for the constant thickness case, is independent of the density of the halfspace $\rho$ and is not influenced by the stress gradients $k_{1}$ and $k_{3}$ in the overburden. If both eigenvalues satisfy $\operatorname{Re}\left(\Lambda_{0}\right)<0$. stability with respect to short-wavelength perturbations is ensured.

Of interest here is the fact that for $\Lambda_{0}=0$ the condition Det $\left(S_{x / 1}\right)=0$ in (91) gives the relation that the incremental moduli have to satisfy for a stress-induced surface buckling at $\zeta=0$ (B1OT, 1965).

3.2.2. Perturbation wih fixed watength $(\Delta \rho g G \omega=$ const $)$. As for the case of the fixed layer thickness, the starting point for the study of the stability of an overburden whose height is much larger than the wavelength of the perturbation is the Euler Lagrange equations given by $(82)$. The new boundary conditions. valid for constant values of $\Delta \rho g G(0$, read

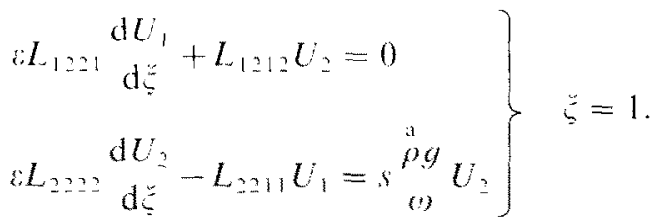

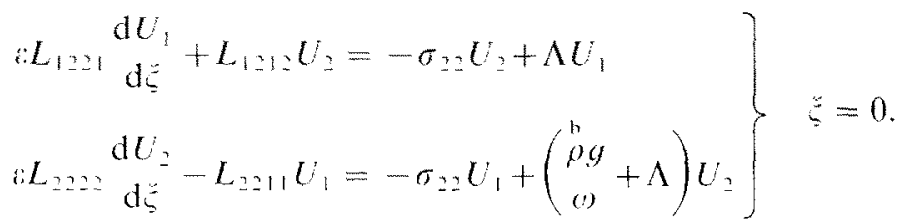

It follows from the definition (27) of the stresses, that for fixed $\omega$, the stress state in the layer is a function of the small parameter $:(a \equiv 1 /(\alpha H)$. Notice that as the layer thickness increases $(H \rightarrow \infty)$, the normal stress at the interface tends to infinity $\left[\sigma_{2}(0)=-\rho g H=-p g / z(x) \rightarrow-x\right]$. This singularity of the stress state at the interface causes difficulties in the asymptotic stability analysis for the determination of $\Lambda$. More specifically, it can be shown that a rigid body mode corresponding to an infinitesimal rotation is the leading term in the expansion of $U_{i}$ as the $\sigma_{22}$ stress at the interface approaches infinity. To correct this anomaly and to compare the present asymptotic results for large overburden thicknesses $(H \rightarrow \infty)$ to those of TRIANTAFYlLIDIS and LIHNER (1993) for the gravitational instability of a solidfluid interface. the pressure in the fluid has to be changed so that a zero normal stress at the interface is obtained. To do this, only the normal stress component $\sigma_{22}$ of (27) has to be altered by adding a constant. The full stress field now reads

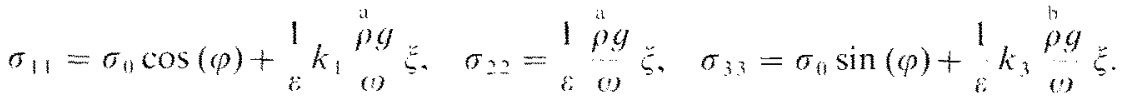


The substitution of $(94)$ in (21) and the remarks made about (22) and (25) that $\mathscr{L}_{i j k}$ are bounded functions of the stresses (i.e. $\mathscr{L}_{i i k l} \rightarrow \mathscr{L}_{i j k l}$ as $\varepsilon \rightarrow 0$ or equivalently as $\left.\left|\sigma_{m i}\right| \rightarrow \infty\right)$, yield the following asymptotic expansions for the nonzero components of the incremental moduli $L_{i j k /}(\xi, 8)$ :

$$
\begin{aligned}
& L_{1111}=-{ }_{\varepsilon}^{1} k_{1} \cdot \stackrel{a}{\rho g} \xi+\stackrel{0}{L}_{1111}(\xi)+\varepsilon L_{1111}(\xi)+\varepsilon\left(\varepsilon^{2}\right) ;
\end{aligned}
$$

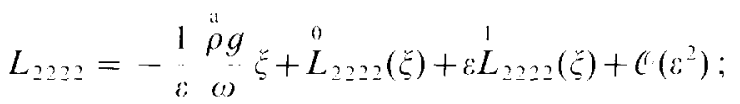

$$
\begin{aligned}
& L_{1122}=\stackrel{0}{L}_{1122}(\xi)+\delta \stackrel{1}{L}_{1122}(\zeta)+C\left(\varepsilon^{2}\right) \\
& L_{2211}=\stackrel{0}{L}{ }_{2211}(\xi)+c \stackrel{0}{L}{ }_{2211}(\xi)+C\left(\varepsilon^{2}\right) ; \\
& L_{1212}=-\frac{1 \stackrel{a}{\rho} g}{\varepsilon} \frac{1}{20}\left(1+k_{1}\right) \xi+\stackrel{0}{L_{1212}}(\xi)+\varepsilon L_{1212}(\xi)+C\left(\varepsilon^{2}\right)=L_{2121} \\
& L_{1221}=\frac{1}{\varepsilon} \frac{a}{2 \omega}\left(1-k_{1}\right) \xi+\stackrel{0}{L_{1221}}(\xi)+\varepsilon L_{1221}(\xi)+c\left(\varepsilon^{2}\right) ;
\end{aligned}
$$

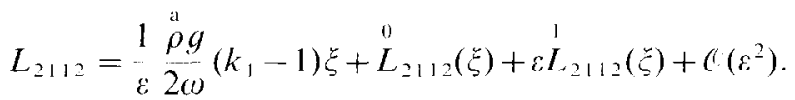

The WKB asymptotic expansions for $U_{i}$ and $\Lambda$ are similar to those of (85). Substitution of (85) and (95) into (82) gives, for the lowest order in $\varepsilon$ [i.e. the $(1)$ terms], two sets of equations, each consisting of two equations for the two unk nowns $U_{i}^{x}(\xi),(\alpha=1,2$; $i=1,2$ ). The resulting system, evaluated at $\xi=0$, is

$$
\begin{aligned}
& {\left[-\stackrel{0}{L}_{1221}(0)\left(\frac{\mathrm{d} f_{x}}{\mathrm{~d} \xi}\right)^{2}-\frac{\ddot{\rho} g}{2 \omega}\left(\mathrm{I}-k_{1}\right) \frac{\mathrm{d} f_{x}}{\mathrm{~d} \xi}+\stackrel{0}{\zeta}_{1111}(0)\right] \stackrel{0}{U}_{1}^{x}(0)} \\
& +\left[-\left(\stackrel{0}{L_{1212}}(0)+\stackrel{0}{L}_{1122}(0)\right) \frac{\stackrel{0}{d}_{x}}{\mathrm{~d} \xi}+\frac{\stackrel{a}{\rho} \mathcal{G}}{2 \omega}\left(1+k_{1}\right)\right] \stackrel{0}{U_{2}^{\alpha}}(0)=0, \\
& {\left[\left(\begin{array}{l}
0 \\
L_{2211}(0)+\stackrel{0}{L}_{2121}(0)
\end{array}\right) \frac{{ }^{0} f_{x}}{\mathrm{~d} \bar{\xi}}\right]{\stackrel{U}{U_{1}^{\alpha}}}^{0}(0)} \\
& +\left[-\stackrel{0}{L}_{2222}(0)\left(\frac{\mathrm{d} f_{x}}{\mathrm{~d} \xi}\right)^{2}+\frac{\mathrm{a} g}{\omega} \frac{\mathrm{d} f_{x}}{\mathrm{~d} \xi}+\stackrel{0}{L}_{2112}(0)\right] \stackrel{0}{U_{2}^{x}}(0)=0 .
\end{aligned}
$$

The corresponding $\mathscr{C}(1)$ term resulting from the substitution of (85) and (95) into the boundary conditions (93) is given by 


$$
\begin{aligned}
& \sum_{x=1}^{2}\left[\left(\begin{array}{l}
0 \\
L_{1221}(0) \frac{d f_{x}}{d \xi}-\Lambda_{0}
\end{array}\right) \stackrel{0}{U}_{1}^{x}(0)+\stackrel{0}{L}_{1212}(0) \stackrel{0}{U_{2}^{\alpha}}(0)\right]=0 \text {, } \\
& \sum_{x=1}^{2}\left[-\stackrel{0}{L}_{2211}(0) \stackrel{0}{U}_{1}^{x}(0)+\left(\ddot{L}_{2222}(0) \frac{\mathrm{d} f_{x}}{\mathrm{~d} \xi}-\frac{\stackrel{b}{\rho}}{\omega}-\Lambda_{0}\right) \stackrel{0}{U}_{2}^{x}(0)\right]=0 \text {, }
\end{aligned}
$$

in which the condition $\sigma_{22}(0)=0$ is accounted for. The leading term $\Lambda_{0}$ in the expansion of the stability eigenvalue is found from (96) and (97) as follows. From (96) one gets a relation between $U_{1}^{\alpha}(0)$ and $U_{2}^{\alpha}(0)(\alpha=1,2)$ :

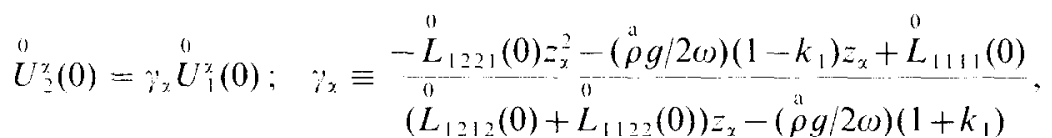

where $z_{x}$, defined as $\left(\mathrm{d} f_{x} / \mathrm{d} \xi\right)(0)$, are the two roots with the negative real part $\left(\operatorname{Re}\left(z_{x}\right)<0\right)$ of the quartic in $z_{x}$

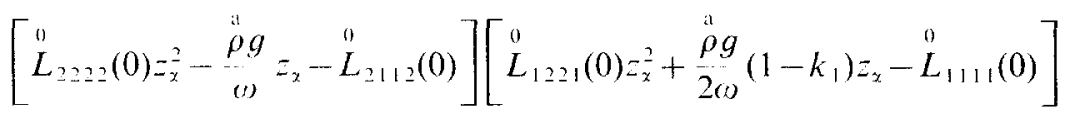

$$
\begin{aligned}
& +\left[\left(\stackrel{0}{\left.L_{1212}(0)+\stackrel{0}{L}_{1122}(0)\right) z_{\alpha}-}-\frac{\ddot{p} g}{2 \theta}\left(1+k_{1}\right)\right]\left[\begin{array}{l}
0 \\
\left(L_{2211}(0)+\stackrel{0}{L}_{2121}(0)\right) z_{\alpha}
\end{array}\right]=0\right. \text {. }
\end{aligned}
$$

The requirement $\operatorname{Re}\left(z_{x}\right)<0$ is dictated by our interest in solutions that decay away from the interface. However, there is no guarantee that such roots exist in the elliptic regime of the deformation of the overburden in view of the presence of terms of the type $\stackrel{a}{\rho} g / \omega$ in $(99)$.

The relations $(90)$, which are still valid in this case, are substituted into (97) to obtain the following $2 \times 2$ system for $U_{1}(0,0)$ and $U_{2}(0,0)$ :

$$
\begin{aligned}
& \sum_{\beta=1}^{2}\left(S_{x \beta}-\Lambda_{0} \delta_{x \beta}\right) U_{\beta}(0,0)=0 \\
& S_{11} \equiv \stackrel{0}{L}_{1221}(0) \stackrel{\begin{array}{c}
z_{1} \hat{\gamma}_{2}-z_{2} \hat{\gamma}_{1} \\
\gamma_{2}-\gamma_{1}
\end{array}}{ }, \quad S_{12} \equiv \stackrel{0}{L}_{1221}(0) \frac{z_{2}-z_{1}}{\gamma_{2}-\gamma_{1}}+\ddot{L}_{1212}(0),
\end{aligned}
$$

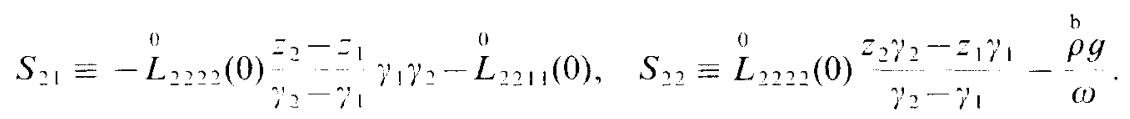

From (100) it follows that $\Lambda_{0}$ is the eigenvalue of the matrix $S_{\alpha \beta}$. It is of interest to note that, when $\Lambda_{0}=0$, the condition $\operatorname{Det}\left(S_{x \beta}\right)=0$ gives the interface instability condition found in TRIANTAFYLLIDIS and LEHNER (1993). It is also worth mentioning that in contrast to the results (91) obtained for the fixed layer thickness, the stability of the overburden depends on both $\stackrel{a}{\rho}$ and $\stackrel{b}{\rho}$ as well as on the stress gradient in the overburden $k_{1}$. 


\subsection{Absence of gravity $(\Delta \rho g H / G=\Delta \rho g / G \omega=0)$}

Finally, attention is turned to the stability of the geological structure when gravity is disregarded ( $g=0$ and hence $\Delta \rho g H / G=\Delta \rho g / G \omega=0$ ). The starting point is once again the variational statement of the stability problem (20) but this time the stresses (27) and the incremental moduli are constant through the overburden thickness because of the absence of gravity. The following Euler-Lagrange equations and boundary conditions are then obtained:

$$
\left.\begin{array}{c}
L_{1221}\left(\frac{1}{\omega H}\right)^{2} \frac{\mathrm{d}^{2} U_{1}}{\mathrm{~d} \xi^{2}}+\left(L_{1212}+L_{1122}\right) \frac{1}{\omega H} \frac{\mathrm{d} U_{2}}{\mathrm{~d} \xi}-L_{1111} U_{1}=0 \\
L_{2222}\left(\frac{1}{\omega H}\right)^{2} \frac{\mathrm{d}^{2} U_{2}}{\mathrm{~d} \xi^{2}}-\left(L_{2211}+L_{2121}\right) \frac{1}{\omega H} \frac{\mathrm{d} U_{1}}{\mathrm{~d} \xi}-L_{2112} U_{2}=0
\end{array}\right\} \quad \forall \xi \in[0,1]
$$

The linear system of equations with constant coefficients (101) admits of the following solution for the eigenmode $U_{i}(\xi)$

$$
U_{i}(\xi)=\sum_{x=1}^{4} V_{i}^{x} \exp \left(\omega H z_{x} \xi\right), \quad \forall \xi \in[0,1]
$$

The constants $\stackrel{x}{V_{i}}$ and $z_{\alpha}$ in (104) $(\alpha=1,2,3,4)$, satisfy the following linear algebraic system resulting from the substitution of (104) into (101):

$$
\begin{aligned}
& \sum_{j=1}^{2} C_{i j}\left(z_{x}\right) \stackrel{x}{V_{i}}=0 ; \\
& C_{11}\left(z_{x}\right) \equiv L_{1221} z_{\alpha}^{2}-L_{1111}, \quad C_{12}\left(z_{\alpha}\right) \equiv\left(L_{1212}+L_{1122}\right) z_{x} ; \\
& C_{21}\left(z_{\alpha}\right) \equiv-\left(L_{2121}+L_{2211}\right) z_{x}, \quad C_{22}\left(z_{\alpha}\right) \equiv L_{2222} z_{\alpha}^{2}-L_{2112} .
\end{aligned}
$$

The linear system in (105) has a nontrivial solution for $\stackrel{x}{V_{i}}$ if and only if its determinant vanishes - a condition that gives the $z_{x}$ as the four roots of the following fourth order (biquadratic) polynomial in $z_{x}$ : 


$$
\left[L_{2222=\frac{2}{y}}-L_{2112}\right]\left[L_{1221=\frac{2}{2}}-L_{1111}\right]+\left[L_{1122}+L_{1212}\right]\left[L_{2211}+L_{2121}\right]=\frac{2}{y}=0 \text {. }
$$

Nolice from the linear system (105) that

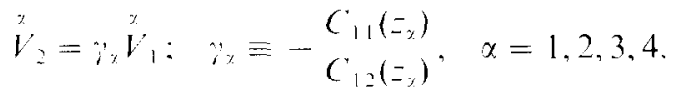

With the help of (107), one obtains, by substituting (104) into the boundary conditions (102) and (103), the following $4 \times 4$ homogeneous linear system for $V_{i}$ $(\% . \beta=1.2 .3 .4)$ :

$$
\begin{aligned}
& \sum_{x=1}^{+} M_{\beta i}(\Lambda){ }^{*}{ }_{1}=0 \\
& M_{1 \%}=\left(L_{1221 z \gamma}+L_{1212 i \gamma}\right) \exp \left(\omega H z_{2}\right) \text {; } \\
& M_{2 \%} \equiv\left(L_{222 z z_{x i \gamma}}-L_{2111}\right) \exp \left(\omega H_{z_{x}}\right) \text {; } \\
& M_{3 \%} \equiv L_{1221} z_{\gamma}+L_{1212 \hat{\gamma}}-\Lambda \text {; } \\
& M_{4 \%} \equiv L_{2222 \% \gamma}-L_{2211}-\Lambda_{i \gamma} \text {. }
\end{aligned}
$$

The desired stability exponent $\Lambda$ is found from a nontrivial solution $\left(V_{1} \neq 0\right)$ for (108). Such al solution implies that $\operatorname{Det}\left[M_{x \beta}(\Lambda)\right]=0$, which after some rather lengthy but straightforward calculations. can be cast in the equivalent form of an easily solvable second-order polynomial in $\Lambda$ with known coefficients. Stability of the overburden is implied when both eigenvalues $\Lambda$ have a negative real part.

\subsection{Conchuding remarks on the special cases}

This section was devoted to the presentation of asymptotic results obtained in the long- $(\omega H \rightarrow 0)$ and short- $(\omega H \rightarrow \infty)$ wavelength limit. In the former case and in the absence of erosion. we found that the stability limit was governed by the slope of the force-displacement response of the overburden. A slope that changes from a positive to a negative value implies that the system goes from stable to unstable, assuming the wavelength ' $)$ of the perturbation is kept constant. Note however, that the same asymptotic analysis conducted for a constant thickness $H$ of the overburden yields a slightly different result. A change from stable to unstable then occurs at a positive value of the slope of the force displacement response equal to, approximately, the absolute value of the lithostatic pressure at the interface. Despite this small difference between the two cases, we expect our geological structure to be stable in general with respect to long-wavelength perturbations. as long as the in situ stresses lead to a positive slope of the force-displacement response of the overburden. If the system 's top surface is allowed to undergo redistribution or erosion, this picture is completely different: the system is unstable with respect to long-wavelength perturbations regardless of the prevailing in situ stress condition or whether (1) or $H$ are kept constant during the asymptotic analysis.

These two valuable results permit one to find the stability condition along the horizontal axis of the o $H$ versus $\sigma_{0}$ plots presented in the next section, depending on 
the exact nature of the boundary condition at the top of the overburden. However, they do not provide information on the locus of the neutral stability curves on these plots. Such information was obtained here, again using asymptotic methods, for longwavelength $(\omega H \rightarrow 0)$ and constant values of $\omega$. Once simplified to the case of a linear, isotropic response for the overburden, we were able to compare our results with those obtained by Ramberg and Stephansson (1964). Note also that the combined knowledge of the stability condition along the horizontal axis and of the neutral stability curves makes it possible to identify the regions of stability in the $(1) H$ versus $\sigma_{0}$ diagrams.

For short-wavelength perturbations $(\omega H \rightarrow \infty)$, we have obtained implicit expressions for the neutral stability exponent that will be used in the next section to check the validity of the finite-element approximate solution. For fixed overburden thickness, the condition of neutral stability coincides with the interface buckling criterion of BIOT (1965). For the case of fixed $(\omega)$. we were also able to establish a link with the results of TRIANTAFYLLIDIS and LFHNER (1993). It is of note that the results of these two different analyses for the short-wavelength limit do differ. Only in the latter case ( $\omega$ fixed) did we find that the substratum density and the overburden stress gradient parameter $k$, had an influence on the stability criterion.

The final part of this section was devoted to the derivation of an exact analylical result for the stability of the structural system in the absence of gravity. This exact solution is compared with asymptotic and numerical solutions in the next section. The interest in disregarding gravity (thereby setting $\Delta \rho g / G \omega=\Delta \rho g H / G=0$ ) originates from the remark that, for the physical systems of geological interest, the values of the two dimensionless numbers $\Delta \rho g / G \omega$ and $\Delta \rho g H / G$ are very small compared to unity.

\section{Results and Discussion}

\section{I. Material parameter selection and F.E.M. procedure}

The general problem formulation presented in Section 2 and the special cases examined in Section 3 are valid for any choice of constitutive equation for the overburden. Once a constitutive law for the top layer is selected, one determines the incremental moduli in (20) required for the analysis of the problem in terms of the known material constants, the stress state in the layer [see (27)] and the values of the internal variables. The Rudnicki and Rice model adopted for the present calculations is a finite-strain generalization - based on either flow-theory or deformation-theory -of the pressure sensitive isotropic hardening model of Drucker and Prager. The relevance of the deformation-theory version of the model to the present work has been explained in Section 2. This model depends on a single internal variable, the accumulated equivalent plastic strain $\eta^{\mathrm{p}}$, which determines the size of the current yield surface. From the perspective of the present study, the Rudnicki- Rice model has two advantages. It has all the required features for a realistic representation of the rate-inde pendent, frictional. geological material of the overburden, e.g. pressure sensitivity, non-associated flow rule and stress-dependent hardening. At the same time, it maintains a reasonable degree of simplicity, e.g. isotropy, constant friction angle and plastic 


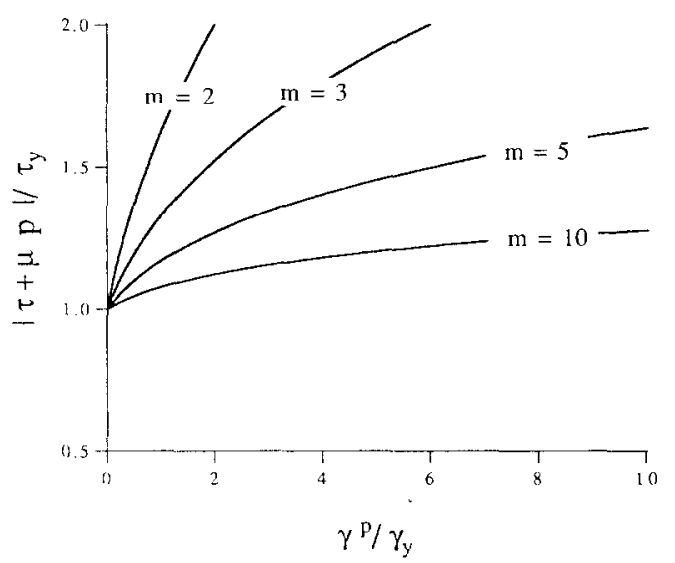

Fig. 2. Dependence of the isotropic yield surface radius $f\left(\gamma^{\beta}\right)=\tau+\mu p$, on the accumulated plastic strain $\because "$ (109). The coefficient $m$ is the hardening cxponent $(m \geqslant 1)$. For $m=1$, the material is clastic; in the limit of $m$ tending to $+x$ the response is elastic, perfectly plastic.

dilatancy. This model was briefly described at the end of Section 2 and provides the functional dependence of the incremental moduli on the stress state in the layer.

In the absence of friction, the uniaxial response of the overburden material was taken to be a simple piecewise power law, the choice being partly motivated by the possibility of comparison of the present results to those due to TRIANTAFYLLIDIS and LEHNER (1993). The relation between the accumulated equivalent plastic strain $\gamma^{p}$ and the radius $f$ of the yield surface is taken to be (see Fig. 2)

$$
\because y=\left\{\begin{array}{cl}
0 & f \leqslant \tau_{y} \\
\left(\frac{f}{\tau_{y}}\right)^{m}-\frac{f}{\tau_{y}} & f \geqslant \tau_{y}
\end{array},\right.
$$

where the hardening exponent $m>1$, and $\tau_{y}, \gamma_{y}$ are the yield stress and strain, respectively, in a pure shear test $\left(\tau_{y}=G \gamma_{y}\right)$. Based on (109), the hardening and secant moduli $h\left(\gamma^{p}\right), h_{1}\left(\gamma^{p}\right)$ required for the determination of the incremental moduli of the flow- and deformation-theory versions of the model [see (21) -(26)] assume the following form in terms of the equivalent shear stress $\tau$ and pressure $p$ :

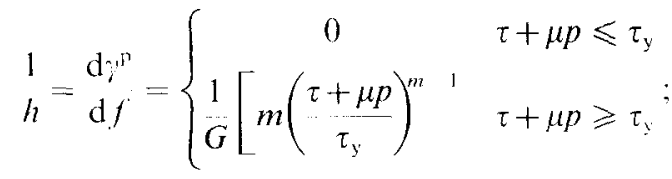

$$
\begin{aligned}
& \frac{1}{h_{1}}={ }^{i}{ }_{\tau}=\left\{\begin{array}{cc}
0 & \tau+\mu p \leqslant \tau_{y} \\
\frac{1}{G}\left[\left(\frac{\tau+\mu p}{\tau_{\mathrm{y}}}\right)^{m}-\frac{\tau+\mu p}{\tau_{\mathrm{y}}}\right] /\left(\begin{array}{c}
\tau \\
\tau_{\mathrm{y}}
\end{array}\right) & \tau+\mu p \geqslant \tau_{\mathrm{y}}
\end{array} .\right.
\end{aligned}
$$


In all the results reported here, except for Fig. $11, \tau_{\mathrm{y}}=10^{-3} E$. The elastic properties of the overburden were taken to be constant for all calculations, with a shear modulus $G=4.17 \times 10^{10} \mathrm{~Pa}$ and a bulk modulus $K=(4 / 3) G$, which corresponds to a Poisson ratio $v=0.2$. The friction coefficient $\mu$ is taken to be in the range $[0,1]$ while plastic dilatancy is disregarded $(\beta=0)$.

The densities of the solid overburden and the viscous half-space are taken to be $\stackrel{a}{\rho}=2.5 \times 10^{3} \mathrm{~kg} \mathrm{~m}^{-3}$ and $\stackrel{b}{\rho}=2.2 \times 10^{3} \mathrm{~kg} \mathrm{~m}^{-3}$ which represent typical densities for sediments and salt. The overburden thickness is varied from $10^{2}$ to $10^{4} \mathrm{~m}$, thus giving a range for the dimensionless parameter $\Delta \rho g H / G$ of between $7.06 \times 10^{-6}$ and $7.06 \times 10^{-4}$. If one considers disturbances with wavelengths of up to $3 \times 10^{4} \mathrm{~m}$, the maximum value of the dimensionless parameter $\Delta \rho g / G \omega$ is approximately $3.35 \times 10^{-4}$. Of course, the values of these parameters can be higher for appropriate combinations of lower shear moduli or higher density differences, overburden thicknesses or disturbance wavelengths. Nevertheless, the above proposed values are thought to be representative of the geological problems of interest.

The numerical calculations of the eigenvalue $\Lambda$ that characterizes the stability of the system are based on a finite-element discretization of the variational form of the problem given in (20). The interval $[0,1]$, which is the domain of definition of the unknown eigenmode whose components are $U_{1}(\xi)$ and $U_{2}(\xi)$, is subdivided in $N$ elements. The elements can be of variable thickness. This feature is dictated by the need for a mesh refinement near the interface $(\xi=0)$ to capture the exponentially decaying interface modes. For these cases of boundary-layer-type modes associated with high values of the dimensionless wave number (refinement is required for $\omega H>5$ approximately), a log-type mesh refinement is employed near the boundary $\xi=0$. For $0 \leqslant \omega H \leqslant 5$ a uniform mesh with $N=5$ elements gives the desired stability parameter within an error estimated to be less than $10^{-3}$. A three node quadratic interpolation function is used for each of the two unknown fields $U_{1}(\xi)$ and $U_{2}(\xi)$, which satisfies the $C^{0}$ (minimum continuity) requirement for the trial fields. A threepoint Gaussian quadrature, which integrates the element stiffness matrices exactly in the case of constant incremental moduli, is employed in all the numerical calculations.

A particularly simple way of calculating the eigenvalue $\Lambda$ for the discretized problem is possible because $\Lambda$ appears only in terms that are evaluated on the interface. The discretized form of the eigenvalue problem in $(20)$ is

$$
[K][U]=0 \Leftrightarrow\left[\begin{array}{ll}
{\left[K_{i i}\right],} & {\left[K_{i]}\right]} \\
{\left[K_{l i}\right],} & {\left[K_{l i}\right]}
\end{array}\right]\left[\begin{array}{c}
U_{i} \\
U_{l}
\end{array}\right]=\Lambda\left[\begin{array}{c}
U_{i} \\
{[0]}
\end{array}\right],
$$

where $\left[U_{i}\right]=\left[U_{1}(0), U_{2}(0)\right]^{\mathrm{T}}$ is the displacement vector for the $\xi=0$ interface and $\left[U_{l}\right]$ is the vector containing the remaining degrees of freedom. The above partitioning of the stiffness matrix $[K]$ permits rewriting (112) in the form

$$
\left[K^{\prime}\right]\left[U_{i}\right]=\Lambda\left[U_{i}\right], \quad\left[K^{\prime}\right] \equiv\left[K_{i i}\right]-\left[K_{i l}\right]\left[K_{l l}\right]^{-1}\left[K_{l i}\right]
$$

from which $\Lambda$ is easily found as the eigenvalue with the highest real part of the $2 \times 2$ matrix $\left[K^{\prime}\right]$. The determination of the neutral stability curves in the $\omega H$ vs $\sigma_{0} / G$ diagrams is based on a simple bisection method for solving Det $\left[K^{\prime}\left(\omega H, \sigma_{0} / G\right)\right]=0$ 
(for real $\Lambda$ ) or $\operatorname{Tr}\left[K^{\prime}\left(\omega H . \sigma_{0}, G\right)\right]=0$ (for complex $\Lambda$ ), in which $\omega H$ is usually held fixed and $\sigma_{0} / G$ varies.

\subsection{Discussion of results}

The results of this study will be presented in three parts. In the first one (Figs 3-6) the calculations are based on the flow-theory model for which we analyse the influence of hardening and friction on the stability of the overburden. The second part (Figs 7 8) compares the flow-and deformation-theories for a material with a power-law type uniaxial response. In the third part (Figs 9 14) the predictions are based solely on the deformation-theory constitutive model. The influence of material parameters, the stress orientation angle, the stress gradient as well as the influence of the redistribution condition at the free surface are studied in this last part. For the sake of completeness, results are presented for negative as well as positive values of the lateral stress parameter $\sigma_{0}$. although the validity of the Rudnicki Rice constitutive model in tension remains an open question.

(a) Flon-therory calculations: effects of grawit, firction and hardening. We shall begin by comparing the stability results that are predicted by a flow-theory with constant hardening $(h=0.36)$ and associated llow-rule $(\mu=\beta=0)$ in the absence and presence of gravity, as shown respectively in Figs 3 and 4 . The layout of most of the subsequent figures will be the same as these two. The coordinates in Figs $3-6$ are the dimensionless wave number $\omega H$ and the dimensionless stress $\sigma_{0} G$. The curves plotted correspond to a state of neutral stability and partition the graphs into regions of stability and instability as indicated by the minus and plus signs, respectively. Figure 4 and the insert in Fig. 3 are calculated for constant values of the dimensionless numbers $\Delta p g(G(1)$ or $\Delta \rho y H G$. As discussed at the beginning of Section 3, the neutralstability curve constructed for a constant $\Delta \rho, g H G$ provides the critical wave number (a) (or, equivalently, the critical wavelength $L_{\mathrm{c}}=2 \pi / \omega_{\mathrm{c}}$ ) of the perturbation that is activated at a given stress. By considering constant values of $\Delta \rho g$ ( $i(\omega$, one actually keeps constant the wave number of the perturbation and the corresponding neutralstability curve gives the critical thickness $H_{1}$ at which the overburden is destabilized by a given stress.

The neutral-stability curves in the absence of gravity, which are depicted by solid lines in Fig. 3. are calculated using the analytical solution presented in Section 3.3. Results for the compressive and the tensile range of deformation are shown in Figs 3 (a) and (b). respectively. The presence of several neutral-stability curves in these graphs is due to the existence of different modes of instability for a given lateral stress a phenomenon often observed in the stability of nonlinear layered solids (see. for example. Dorris and Nimat-Nasser, 1980: Triavtafyllidis and Maker, 1985: STEIr, 1986). For simplicity, only the parts of the neutral-stability curves that provide the smallest (in absolute value) critical stress are depicted in subsequent figures. Notice that the neutral-stability curves associated with the various modes merge for large cnough values of $(n) H$, both in tension and in compression. The existence of a vertical asymptote for these curves is expected from the limit analysis in Section 3.2 that gives the critical stress as (i) $I \rightarrow+x$. It is found that for values of (o) $H$ of the order of 20 
(a)

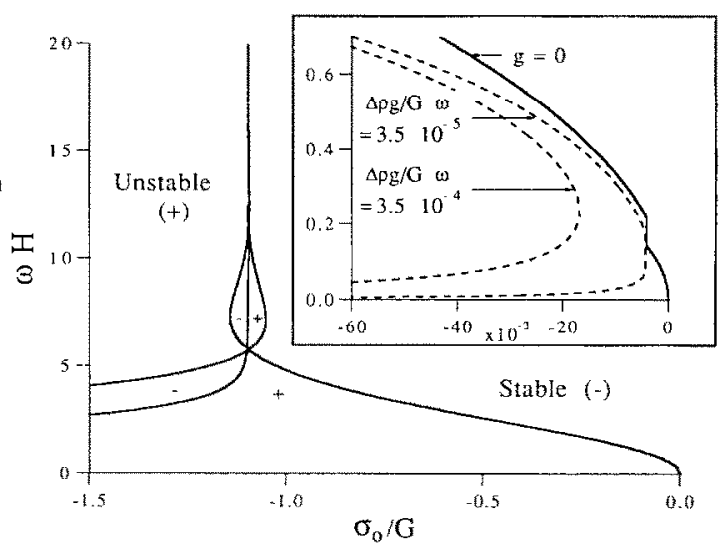

(b)

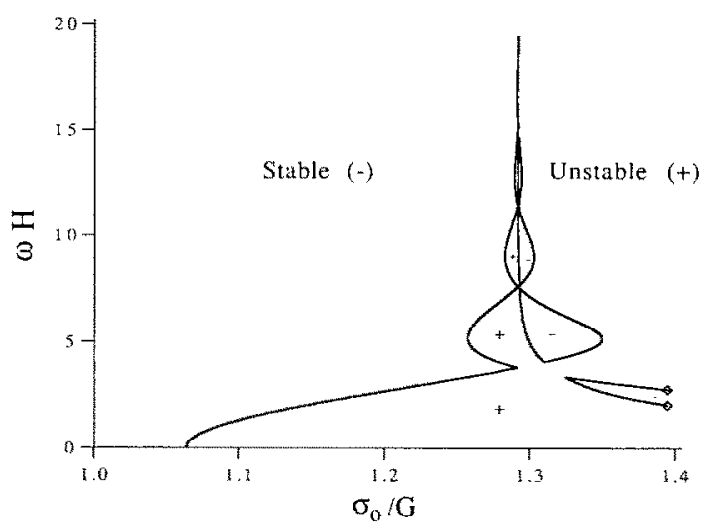

FIC, 3. Stability diagrams and neutral-stability curves (solid lines), in the absence of gravity in a space spanned by the dimensionless wave number $\omega H$ and the dimensionless stress $\sigma_{0} G$, under (a) compression and (b) tension. In the insert the results are compared with those obtained in the presence of gravity with an asymplotic method for small of $H$ (dashed lines). The diamonds in the tensile regime indicate loss of cllipticity. These curves are based on flow theory with a hardening parameter $h=0.36$ and the associated llow rule $\mu=\beta=0$.

and higher, the difference between the exact value of critical stress and the one obtained from the asymptotic analysis is negligible.

As will be noted in Fig. 3(a), in the compressive regime the solid curve $(g=0)$ crosses the origin of the graph. This behaviour is to be expected since, in the absence of gravity and for long-wavelength perturbations, the overburden behaves like a thin column : the infinitely long column buck les for a vanishingly small applied compressive force. The same curve also has an abrupt change in its slope, as seen in the insert, due to the discontinuous values of the tangent moduli at the yield point of the adopted uniaxial stress -strain curve [see (110)]. For the same reason, i.e. the beam-like behaviour of the overburden for long-wavelength perturbations, a finite stress is required for destabilization of the structure in tension, as seen in Fig. 3(b). The loss of stability under tension corresponds to a maximum force in the response of the overburden to uniaxial plane-stress (zero stress along the $x_{2}$-direction) and plane-strain ( $\angle$ ero strain 
(a)

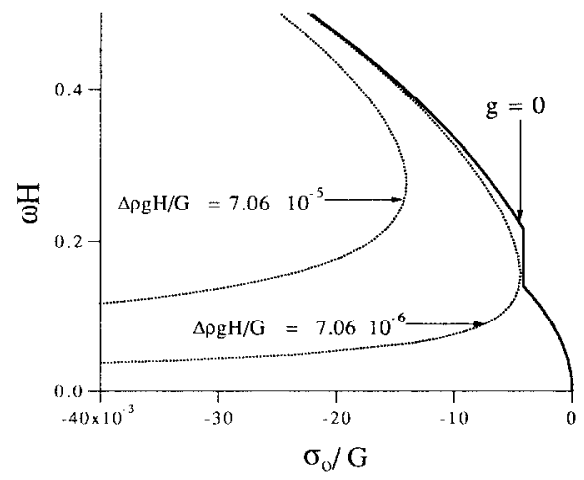

(b)

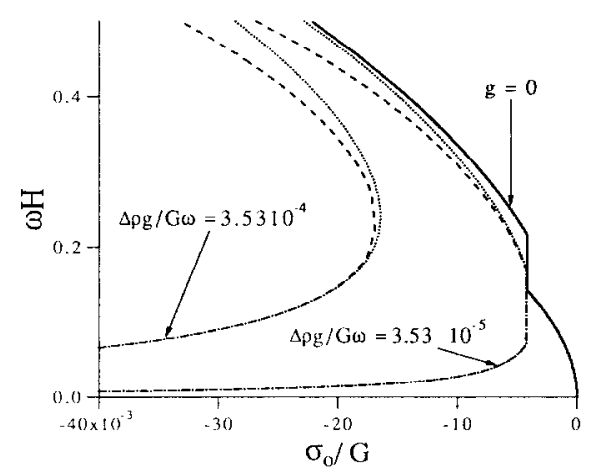

FlG. 4. Comparison, in the compressive regime, between results obtained in the absence of gravily (analytical method: solid lines) and in the presence of gravity (finite-element method: dotted lines; asymptotic analysis for ( $) H$ tending to 0 : dashed lines). Results are for two values of the dimensionless numbers $\Delta \rho g H_{i} G$ (a) and $\Delta \rho g_{i} G(a)$ (b). The values of these two numbers correspond to an overburden thickness of 100 and $1000 \mathrm{~m}$ or a wave number of $2 \times 10^{-4}$ and $2 \times 10^{-3} \mathrm{~m}^{-1}$, respectively. These curves are based on flow theory with a constant hardening parameter $h=0.3 G$ and $\mu=\beta=0$.

along the $x_{3}$-direction), in accordance with Considère's principle. Further increase in the tensile stress will induce the development of a neck-type instability. The diamonds ending the neutral-stability curves in the same graph indicate the loss of ellipticity in the overburden material, which corresponds to the onset of faulting (deformation becomes localized in the form of shear bands). The stress corresponding to the loss of ellipticity under uniaxial plane stress and plane strain tension is much larger than the maximum force because of the relatively high hardening of the material $(h=0.3 G)$ and the fact that a flow theory is being used in the calculations.

Consider now the system's response in the presence of gravity. The results in tension are essentially indistinguishable from those presented in the absence of gravity and were thus omitted. The sameness in behaviour can be explained by the negligibility of the normal stress added by the weight of the overburden compared with the large value of the axial tectonic stress $\sigma_{0}$ (of the order of the modulus of elasticity in shear) that is required to trigger the maximum force (necking) instability in tension [see Fig. $3(b)$ ]. However, there is a significant influence of gravity in the compressive range of deformation, as can be seen by inspection of the insert in Fig. 3(a). The dashed 
neutral-stability curves in the insert were obtained from the asymptotic analysis for small $\omega H$ and for constant values of $\Delta \rho / G \omega$. Note that the dashed curves do not intersect the horizontal axis as discussed in the stability analysis for the beam resting on a fluid at the end of Section 3.1.2. The viscous substratum has a stabilizing influence in the presence of gravity for compressive deformations: the infinitely long beam resting on a "fluid" requires a stress of finite magnitude in order to buckle. In the rest of this paper the minimum (in absolute value) magnitude of this stress will be denoted by $\sigma_{m}$ and the associated wave number by $(\omega H)_{m}$.

For a compressive stress $\sigma_{0}$ larger than the minimum critical stress $\sigma_{\mathrm{m}}$, the system is stable regardless of the overburden thickness. For stresses smaller than this minimum value, there are two critical values of $\omega H$ that define the range of overburden thicknesses or disturbance wavelength for which the top layer is unstable. It is noteworthy that, for the values of the parameters employed in these calculations, the minimum stress $\sigma_{\mathrm{m}}$ occurs at a small value of $(\omega H)_{\mathrm{m}}$ and thus corresponds to a "beamtype" mode of instability. In the rest of this paper and in the interest of simplicity, an instability mode with a small value of $\omega H$ will be referred to as a "beam" mode of instability. Later on, we shall also see cases for which $\sigma_{\mathrm{m}}$ occurs at a large value of $(\omega H)_{\mathrm{m}}$ and thus corresponds to a "surface-type" mode. Another general remark on the presentation of all subsequent results is in order at this point. From here on, the stable and unstable regions in the figures will not be identified as was done in Fig. 3. Since all figures are topologically similar to Fig. 3, it is easy to identify the stable regions of the graphs by keeping in mind that the origin $\left(\omega H, \sigma_{0} / G\right)=(0,0)$ is always stable in the presence of gravity and in the absence of material redistribution at the top surface $(s=0)$.

We continue the discussion of the influence of gravity by considering some additional information: the results of finite-element calculations. which are plotted in dotted lines in Fig. 4. The analytical results in the absence of gravity are represented by solid lines, while the results of the asymptotic analysis for small $\omega H$ and constant values of $\Delta \rho g / G \omega$ by dashed lines, as was done in Fig. 3. Results for fixed values of the overburden thickness are depicted in Fig. 4(a). The two values of dimensionless number $\Delta \rho g H / G$ correspond to layer thicknesses $H$ of 1000 and $100 \mathrm{~m}$. Note again that the slope discontinuity of the solid curve, corresponding to the case of $g=0$, permits one to identify the transition between the elastic and elastoplastic responses of the overburden in the $\left(\omega H, \sigma_{0} / G\right)$ space. The finite-element results in Fig. 4(a) show that a compressive stress in the range of the yield stress is sufficient to destabilize a thin overburden. As the overburden thickness increases, so too does the absolute value of the critical stress $\left|\sigma_{m}\right|$ (now well in the plastic range of the overburden response) and the corresponding wave number $\omega_{\mathrm{m}}$.

A similar trend is observed in Fig. 4(b), which corresponds to fixed values of the perturbation's wave number. The calculations were done for two values of the dimensionless number $\Delta \rho g / G \omega: 3.53 \times 10^{-4}$ and $3.53 \times 10^{-5}$, which correspond to wave numbers $\omega: 2 \times 10^{-4}$ and $2 \times 10^{-3} \mathrm{~m}^{-1}$, respectively. In the former case, the minimum critical stress $\sigma_{\mathrm{m}}$ equals approximately the yield stress and is reached for $(\omega H)_{\mathrm{m}}$ in the range $[0.07,0.18]$, whereas the minimum critical stress is reached at $(\omega H)_{\mathrm{m}}=0.24$ for the second choice of wave number. Overburden thicknesses varying between 35 and $90 \mathrm{~m}$ under a compressive lateral stress equal to the yield stress are 
thus destabilized with a perturbation wavelength of, approximately. $3.1 \mathrm{~km}$. Similarly, a mode of instability with a wavelength of $310 \mathrm{~m}$ is triggered if a $1200 \mathrm{~m}$ thick overburden is compressed at a stress four times the yield stress. The thinner the overburden. the smaller the compressive stress required to reach the first instability and the longer is the first instability's wavelength.

In Fig. 4(b) the finite-element results (in dotted lines) are additionally compared with the asymptotic analysis results (in dashed lines) for small values of oH as discussed in Section 3.1.3. A remarkably good agreement is found between them for values of (1) $H$ up to 0.2 . Interestingly enough, as the agreement between the accurate numerical method and the approximate asymptotic method deteriorates for increasing $(b H$, the finite-element results tend towards the analytical predictions obtained in the absence of gravity. Indeed. the superiority of the finite-element calculations is clear only in the transition interval for $0 H$ of $[0.2,3.0]$. For higher values of $\omega H$, the eflects of gravity tend to be negligible compared to the effects of the prestress in the overburden.

The effects of friction parameter $\mu$ in the stability of the overburden are presented in Fig. 5. The results for constant value of $\Delta p q G(\omega)$, shown in Fig. $5(b)$, were restricted to the compressive range of deformation, since the corresponding results in tension were similar to the ones presented for at constant value of $\Delta \rho, H G$. shown in Fig. $5(a)$. When the value of $\mu$ differs from the value of the dilatancy parameter $\beta$, one obtains a nonsymmetric (non-selfadjoint) stability operator for the problem, thus raising the possibility of complex cigenvalues. A result of importance that holds for all the ealculations reported in this study is that the stability eigenvalues $\Lambda$ are always real in the vicinity of a stability transition. Conscquently, flutter-type instabilities [mentioned, for example, in Rict (1976) and NHblimav (1979)] are never present in this problem. Such instabilities seem physically implausible anyway in the geomechanical stability context of interest here. since they are characterized by an oscillatory evolution in time.

The general structure of the results shown in Fig. 5 is similar to the one presented carlier in Figs 3 and 4 . The shape of the neutral-stability curves for small $a H$ as well as the values of the minimum critical stress $\sigma_{m}$ in compression again correspond to a beam-type mode. In the case of tension, there is a maximum load and the first instability is of a necking type, as was also observed in Figs 3 and 4 . Notice the difference between the effect of friction in tension and in compression. Whereas the influence of friction is minor in compression, a variation of $\mu$ from 0 to 0.6 leads to a $50 \%$ decrease of the tensile critical stress. This difference is due to the dominance of the geometric terms in compression, which are not affected by friction. Furthermore. an increase of the friction coefficient leads to an increase, in absolute value, of the minimum critical compressive stress $\sigma_{m}$. whereas it is accompanied by a decrease of the minimum critical stress in tension. It appears that friction stabilizes the system in compression and destabilizes it in cension. This difference is attributed to the asymmetric contribution of the friction coefficient on the tangent moduli. with respect to the pressure. as revealed in (22).

In Fig. 5(b) the finite-element analysis results in the compressive range (solid curves) are plotted sogether with those of the asymptotic analysis in the limit of small $w H$ (dashed curves). The excellent agreement for o $H$ less than 0.2 , which was first mentioned in the discusion of Fig. $4(b)$, is fully confirmed. Note again that rather shallow 
(a)
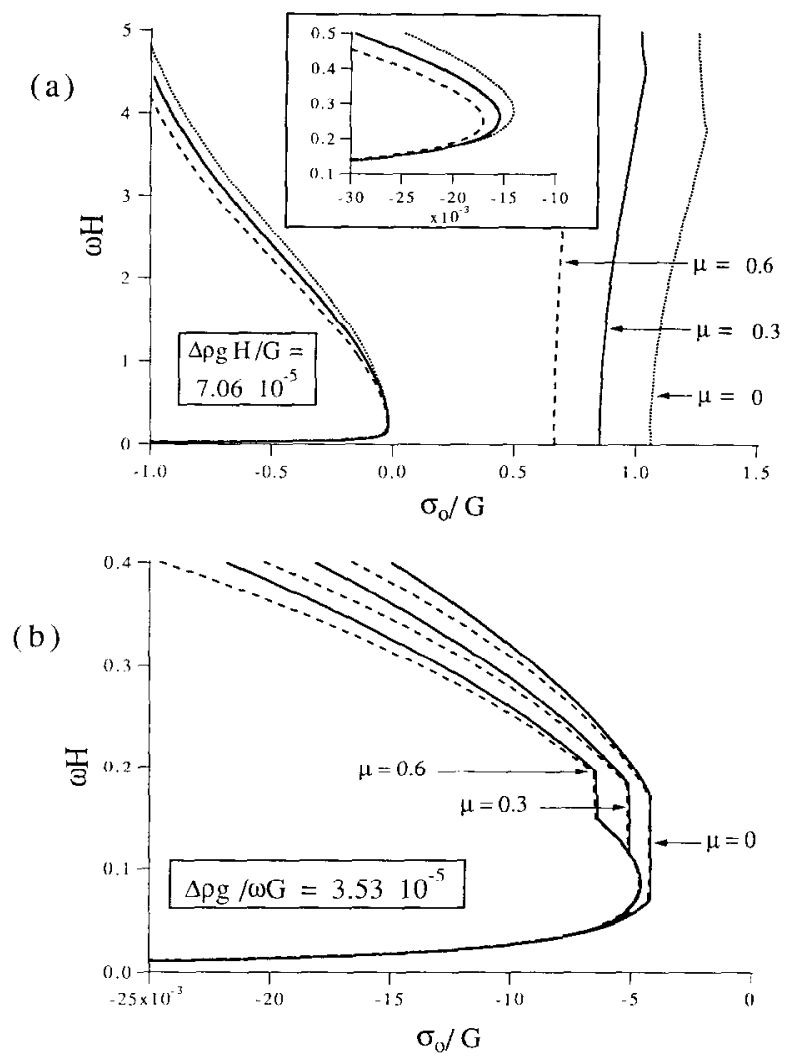

Fic. 5. Influence of the friction coefficient $\mu$. F. E.M. results for a constant value of $\Delta \rho \varphi H$ G (a). Comparison between the F.E.M. results (solid lines) and the asymptotic results for small (oH (dashed lines). for a constant value of $\triangle p g$ grog (b). Note the opposite effect of friction in tension compared with in compression.

These results are based on flow theory with a constant hardening parameter $h=0.3 G$ and $\beta=0$.

overburdens $(\omega H \simeq 0.1-0.2$ corresponding to $H \simeq 50-100 \mathrm{~m})$ are destabilized for a compressive stress of the order of the yield stress.

The investigations based on the flow-theory model are completed with the study of the influence of the hardening parameter $h$, which is presented in Fig. 6 . The finiteelement results, calculated here for a fixed overburden height, show a trend similar to the one observed for the friction coefficient: the minimum critical compressive stress $\sigma_{m}$ is only marginally affected by the hardening parameter $h$ in compression, whereas the minimum stress for instability in tension is drastically reduced as the hardening parameter diminishes. Again, this difference between tension and compression is attributed to the dominance of the geometric effects in compression.

One can thus conclude that, in the context of flow-theory, the friction and hardening parameters have little influence on the minimum critical stress $\sigma_{m}$ in compression. The influence of the same parameters is significant in tension, but the minimum stress for the onset of instability is rather large (of the order of $0.1 G$, for realistic values of $h$ and $\mu$ ). Furthermore, when flow-theory is used (see RUDNICKI and RICE, 1975), the lirst instability is always found to be of a necking type in tension. while the initiation 


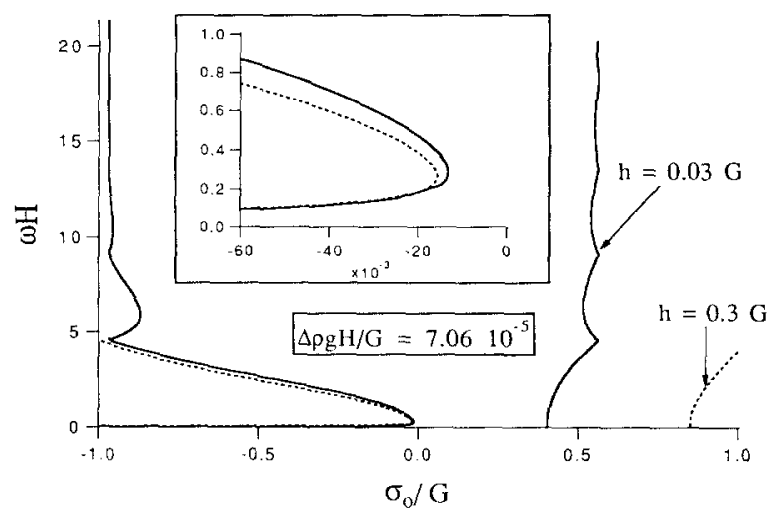

FIG. 6. Influence of the hardening parameter $h$. F.E.M. results for a constant value of $\Delta \rho g H / G$ and two values of $h(0.03 G$ : solid lines: $0.3 G$ : dashed lines). This influence is important in the tensile regime but minor in the compressive one for small $\omega H$. since geometric effects dominate in that range. These results are based on flow theory with $\mu=0.3$ and $\beta=0$.

of faulting is found to occur at unrealistically large stresses in both tension and compression. These results contradict the predictions of laboratory experiments on low-cohesion materials (e.g.. NetTLETon and Eikins, 1947) and question the validity of flow theories for modelling the stability of a brittle layer resting on a viscous substrate. It is the aim of the following part of this discussion to establish a comparison between flow and deformation theories in order to clarify this question.

(b) Comparison heneen flow-and deformation-theory calculations. The comparison between the stability predictions based on flow and deformation theories is presented in Figs 7 and 8. The results are obtained with the power-law hardening function given in (109) and depicted in Fig. 2 for several values of the hardening exponent $m$. The same hardening function will be employed in the rest of this paper. Values for $m$ of 10 and 3 were chosen for the calculations underlying Fig. 7 and Fig. 8, respectively. Deformation theories are known to predict lower (in absolute value) critical loads than equivalent flow theories, i.e. theories with the same response to proportional loading. The incremental moduli of a deformation-theory are lower in a direction perpendicular to that of the proportional loading than the moduli of an equivalent flow theory with a smooth yield surface. This difference explains the lower critical loads usually predicted in stability calculations based on an equivalent deformation theory, since a sudden deviation from proportional loading is frequently the reason for the onset of such an instability. A reduction in stiffness in a direction perpendicular to that of the proportional loading is also found in a class of flow theories that exhibit a vertex at the current loading point of their yield surface. For the case of rock masses that deform by frictional sliding along a randomly oriented set of cracks or smalf faults, RUDNICKI and RICE (1975) have shown the possibility of such a yield vertex and proposed a deformation theory model as a simple way to account for this effect. The importance of the existence of a corner at the yield surface for the prediction of failure in rocks was also established in the above-mentioned work by showing that the proposed deformation-theory model could capture the initiation of faulting under 


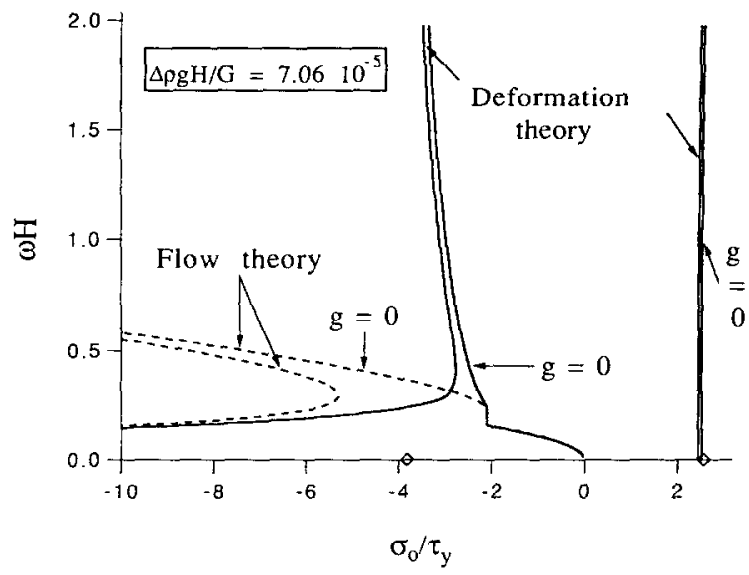

Fig. 7. Comparison between flow (dashed lines) and deformation (solid lines) theories in the absence (analytical results) and in the presence (F.E.M. results) of gravity. The tensile critical stresses predicted by the flow theory, not shown on this graph, are seven times larger than the one obtained with the deformation theory. The diamonds on the horizontal axis indicate loss of ellipticity for the case of the deformation theory. These results were derived assuming a hardening exponent $m=10$ and $\mu=0.3, \beta=0$.

triaxial states of stress-an effect which is not predicted by flow-theory, unless an unrealistic softening is introduced.

Figure 7 depicts the stability results for the deformation- (solid curves) and flow(dashed curves) theories obtained by the finite-element method in the presence of gravity and by the analytical solution in the absence of gravity. The critical-stress predictions of the flow-theory in tension were seven times larger than the results of the deformation theory and have thus been omitted. Moreover, the diamond on the horizontal axis indicates, for the case of the deformation theory, a loss of ellipticity

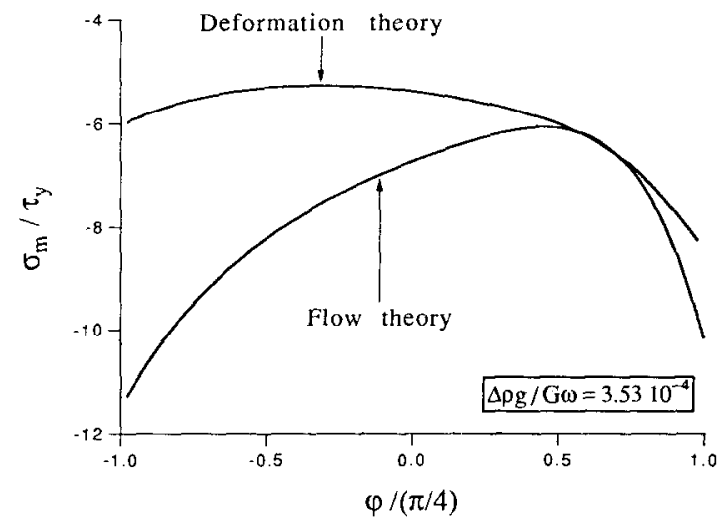

FIC. 8. Influence of the stress orientation angle of on the smallest (in ahsolute value) critical compressive stress $\sigma_{\mathrm{m}}$ required for destabilizing the system at a constant value of $\Delta \rho \mathrm{g} / G \omega$. Comparison between the results of deformation and flow theories based on the asymptotic analysis for small $\omega H$. Note the agreement between the two theories for certain stress orientations. These results are derived for a hardening exponent $m=3$ and $\mu=0.3, \beta=0$. 
which corresponds to the onset of laulting. Notice that the tensile stress at the loss of cllipticity is only slightly larger than the tensile stress at the onset of the neck-type instability. This result suggests that the initiation of an instability in the tensile range of deformation cannot be triggered without faulting in the overburden. Both experimental and field observations suggest that such is indeed the case for multilayered systems (e.g. VINDSVILL.F and JACKSON, 1992).

The difference between the stability predictions of the two theories is also important in compression, despite the dominant role of the geometrical effects. The minimum critical stress $\sigma_{m}$ for the deformation-theory is $50 \%$ less than that of the flow-theory, but the corresponding instability mode is still a beam-type one. Note also the different shape of the neutral-stability curve under compression in the case of the deformationtheory. For a stress $\sigma_{n}$ smaller than $\sigma_{m}$ but larger than the stress associated with the loss of ellipticily in the overburden (at approximately $-4 \tau$, and marked by a diamond in Fig. 7). there exists a large range of wave numbers, corresponding to various types of modes. that destabilize the system. The conditions under which instabilities with a large wave number correspond to the minimum, in absolute valuc. critical stress $\sigma_{m}$ will be investigated below.

Concentrating on small values of $a H$ for the case of compression and for a hardening exponent $m=3$. we have plotted in Fig. 8 the vatriation of $\sigma_{m}$ as a function of the stress orientation angle $(\rho$, introduced in (27), on the basis of the asymptotic analysis in Section 3.1.3. Observe the difference of a factor of 2 between the values of $\sigma_{n}$ predicted by the two theories for $p=-\pi 4$. However, for stress orientations around $\rho=-\pi, 8$, the two theories yield similar predictions. One possible explanation is as follows. A variation in stress orientation implies a change in effective stress $\tau$ and pressure $p[$ see (21)] and thus a modification of the value of the effective plastic strain that controls hardening (see Fig. 2). If the permanent deformation does not clearly exced the elastic strain, the difference between the moduli obtained using flow and deformation theories should be small and the predictions of the two theories should be comparable. A second factor that could explain the small difference between the two theories for certain stress orientations will become clear at a later stage of this discussion.

The main conclusion drawn from the above analysis is that according to deformation-theory, geological systems in compression suffer from structural instabilities of a beam-lype prior to but nof far from the initiation of faulting as lateral stresses increase in magnitude. For the case of tension. a neeking-type instability just precedes the lautting one for relatively thin overburdens. This observation is in agreement with the result of HuL and Hutcinsson (1975), who find the maximum load instability prior to loss of ellipticity in uniformly strained specimens under uniaxial plane strain tension conditions. The aforementioned behaviour in tension ceases to be true for thicker layers, since in such a case stress gradients become important. The closeness between the onset of the first instability in folding or necking and the onset of faulting is. of course, in agreement with field observations and results of laboratory experiments.

(c) Effects of material parameters, stress-state and redistribution condinon. Having established the appropriateness of deformation theories for modelling the stability of the layered structure at hand. we employ exclusively the deformation-theory model 
(a)

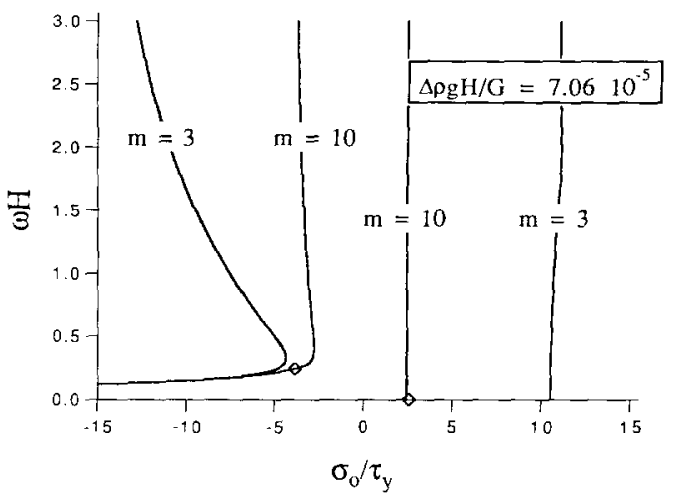

(b)

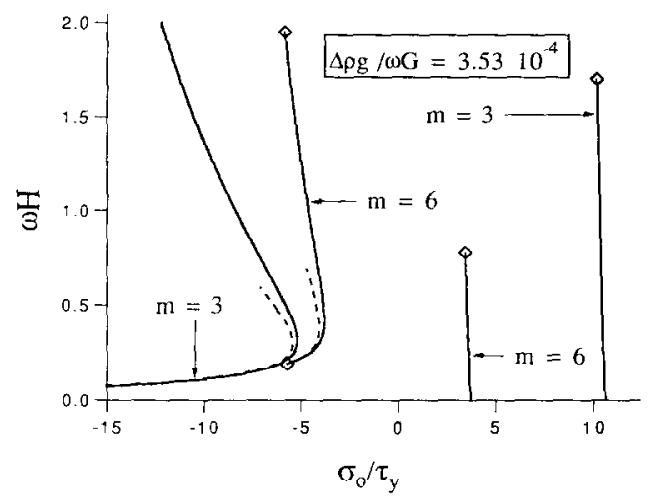

Fig. 9. Influence of the hardening exponent $m$ on the results obtained with deformation theory, for a constant value of $\Delta p g H / G(a)$ and $\Delta p g / G(t)$ (b). The solid curves correspond to the F.E.M. calculations and the dashed ones to the results of the asymptotic analysis for small values of (') $H$. The diamonds indieate loss of ellipticity. For these curves. $\mu=0.3$ and $\beta=0$.

proposed at the end of Section 2 in all the subsequent calculations. The results from our study of the influence of the overburden material properties, namely the hardening exponent $m$ and the cohesion $\tau_{y}$, are given in Figs 9-11. The role of the in situ stress distribution in determining the system's stability is discussed with reference to Figs 12-13, while the study of the erosion or redistribution condition at the top surface is reported in Fig. 14.

The sensitivity of the stability results to the hardening exponent $m$ for constant values of the dimensionless numbers $\Delta \rho g H / G$ and $\Delta \rho g / \omega G$ are depicted in Figs $9(a)$ and $9(\mathrm{~b})$, respectively. This analysis is analogous to the study of the influence of the hardening parameter $h$ shown in Fig. 6 for the case of the flow-theory. Figure 9(a) shows that the hardening exponent $m$ influences the stress at the loss of ellipticity in both tension and compression. For the very low hardening case of $m=10$, the necking (maximum load) instability in tension practically coincides with the initiation of faulting (loss of ellipticity). The instability in compression on the other hand, is triggered prior to the loss of ellipticity: the magnitude of the compressive stress $\sigma_{n}$ must be some $30 \%$ greater than $\sigma_{\mathrm{m}}$ to trigger faulting. For the higher hardening case of $m=3$, the loss of ellipticity requires a stress magnitude that differs significantly 


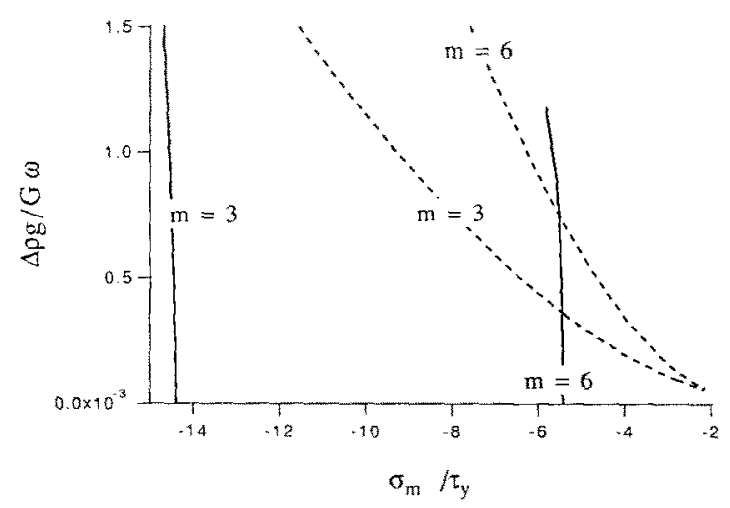

Fic. 10. The smallest (in absolute value) compressive critical stress $\sigma_{m}$ required to destabilize the system as a function of $\Delta p g G(t$. Comparison between the results of the asymptotic analysis for the longwavelength (dashed eurves) and the short-wavelength (solid curves) limits. The deformation theory was considered for two values of the hardening exponent $m$. Note that for $m=6$ and for large cnough values of $\triangle p g^{\prime}\left(G(1)\right.$, the asymptotic analysis for large $(\omega) H$ predicts the smallest stress $\left|\sigma_{\mathrm{tu}}\right|$. These curves were derived for $\mu=0.3$ and $\beta=0$.

from the one necessary for the onset of instability in either tension (maximum load stress) or compression (minimum critical stress). No diamonds marking the loss of ellipticity are shown for the case $m=3$, since they lie outside the range of stress depicted in Fig, 9(a). The same trend with regard to the hardening exponent's effect on the loss-of-ellipticity stress is found in Fig. 9(b), which furthermore shows the reasonably accurate predictions of the asymptotic analysis of Section 3.1 .3 for small (o) $H$ in the compressive range.

A second common feature of the two graphs of Fig. 9 is the influence of the hardening parameter $m$ on the shape of the neutral-stability eurve when the system is under compression. In contrast to the flow-theory results in Fig. 6, there is a large range of the dimensionless wave numbers $\omega H$ corresponding to neutrally stable modes for values of $\sigma_{0}$ slightly smaller than the minimum critical stress $\sigma_{m}$. This is the case, for example, for $m=10$ in Fig. 9(a) and for $m=6$ in Fig. 9(b). This feature raises the question of whether or not a beam mode of instability, which occurs at small values of $\omega H$, will always correspond to the lowest critical stress $\sigma_{m}$. To answer this question, we have plotted in Fig. 10 the dependence of the minimum critical stress in compression $\sigma_{m}$ predicted by the asymptotic analyses for the short-wavelength limit (corresponding to an interface type mode) and the long-wavelength limit (corresponding to a beam-type mode), as a function of the dimensionless number $\Delta \rho g(\omega G$. For a small value of the hardening exponent $(m=3)$, beam modes (long-wavelength limit. dashed curves) always give the lowest (in absolute value) critical stress in compression. However, for another value of the hardening parameter $(m=6)$, the interfacial instabilities (short-wavelength limit, solid curves) are triggered for smaller absolute values of $\sigma_{\mathrm{m}}$, if large enough values of $\Delta \rho y / \omega G$ are considered. This result, obtained for fixed values of the perturbation wave number $(t)$, shows that beam-type modes of instability are dominant for shallow overburdens ( $\omega \mathrm{H}$ small), whereas thick overburdens are destabilized in a mode reminiscent of interfacial instabilities $(\omega H$ large). The results of this calculation provide a rational basis for choosing, according 
to the material properties of the overburden, the magnitude of the top layer"s thickness above which the assumption of infinite layer thickness can be safely adopted. This assumption, introduced for the sake of analytical tractability by TRIANTAFYLLIDIS and LEHNER (1993), is thus shown to be a meaningful one for overburdens with low hardening and for values of $\Delta \rho g / \omega G$ beyond a certain magnitude.

In all the calculations reported so far, the stability of a rather cohesive overburden $\left(\tau_{y} / E=10^{3}\right)$ has been investigated. Even though this ratio appears to be realistic for modelling the lithosphere (SHEMENDA, 1992), one could expect lower values of that ratio. Indeed, sand, which is used as an analogue material in laboratory experiments, has a cohesion of only a few hundred pascals (KRANTZ, 1991). Furthermore, in view of the dependence of the elastic moduli of sand on the confining pressure, we could expect a ratio $\tau_{y} / E$ as low as $10^{-5}$. It is thus reasonable to conclude our materialsensitivity investigation with a study of the influence of cohesion on the stability of the overburden. The results calculated using the finite-element method for the compressive and tensile ranges of deformation and assuming a constant value of the dimensionless number $\Delta \rho g H / G$, chosen on the basis of an overburden thickness of $1000 \mathrm{~m}$, are displayed separately in Figs 11 (a) and (b), respectively. One of the expected results is the shrinkage of the stability region, centred on the elastic domain, for diminishing values of the cohesion. However, an unforeseen result, both in tension and in compression, is the variation in the mode of instability corresponding to the lowest magnitude of the lateral stress. Indeed, a shift of the dominant dimensionless wave number $(\omega H)_{\mathrm{m}}$ from 0.37 to 0.84 can be observed as the cohesion is reduced by a factor of 10--from $E \times 10^{-3}$ to $E \times 10^{-4}$. That increase becomes even greater if one reduces the cohesion further by half: $(\omega H)_{\mathrm{m}}$ takes on a value of 1.12 for a cohesion of $5 E \times 10^{5}$. In tension, faulting in the overburden coincides with a necking-type instability for small values of the cohesion; for the case of more cohesive materials, faulting requires stresses of larger magnilude than those leading to necking. These results are similar to the ones presented in Fig. 9, which illustrates the influence of the overburden hardening exponent $m$ on its stability. We explain this analogy by noting from Fig. 2 that a reduction in the cohesion $\tau_{y}$ leads to an increase of the normalized effective stress and equivalent plastic strain (with $\gamma_{y}=\tau_{y} / G$ ). For a constant effective stress $\tau$ and pressure $p$, the reduction in $\tau_{y}$ implies a displacement of the current position on the stress-strain curve away from the origin of the graph. From the same figure, one can see a similar effect if the value of $m$ is increased.

Having investigated the role of the overburden material properties, we now turn our attention to the influence of the lateral stress gradient in the top layer [see definition in (27)]. Note that for the rest of this section the value $E \times 10^{-3}$ is once again used for the cohesion $\tau_{y}$. This is the same value that has been used in deriving all the results presented in Figs 3-10. The first item studied is the influence of the lateral stress $\sigma_{11}$ gradient $k_{1}$. The results depicted in Fig. 12(a) pertain to the compressive range of deformation and to two different values of the hardening exponent $m$, whereas those depicted in Fig. 12(b) are calculated for the same two values of $m$ but correspond to the tensile range of deformation. The results shown in Fig. 12(c) have been calculated for $m=3$ under tensile conditions but for two values of the dimensionless number $\Delta \rho g H / G$ corresponding to overburden thicknesses of 1 and $10 \mathrm{~km}$. The first two graphs, which correspond to a shallow overburden (1 $\mathrm{km}$ thick), show the weak 
(a)

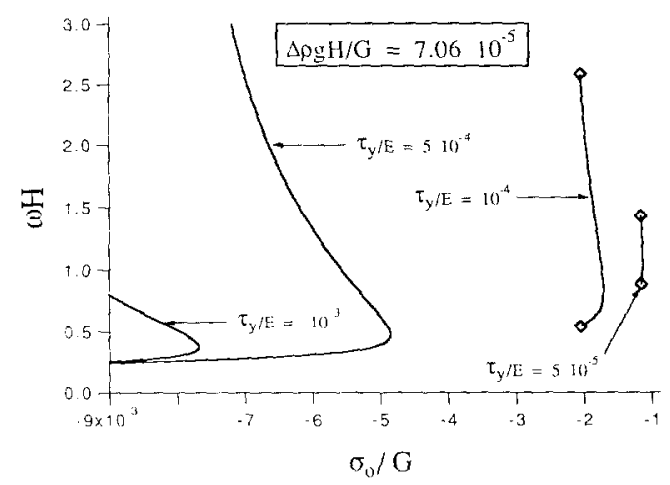

(b)

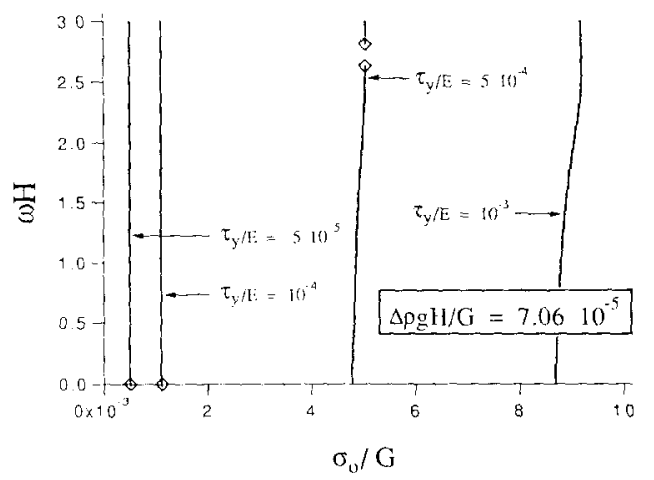

Fre. H. Intluence of the cohesion $\tau_{1}$, aceording to the results of the F. F. M. analysis and for a constant value of $\Lambda p g H: G$ in compression (a) and in lension (b). Note the increase in the dominant wave number $(1 / I)_{m}$ in compression as the coltesion is reduced. Loss of ellipticity (marked by diamonds) is the dominant mode of instability in tension. For small cohesion. These results are based on deformation theory with at hatdening exponent $m=6$ and $\mu=0.3, \beta=0$.

intluence of the in siru stress gradient parameter $k_{1}$ compared with the sensitivity to the hardening exponent. However, for a thick overburden [Fig. 12(c) and $\left.\Delta p g H: G=7,06 \times 10^{+}\right]$and in the tensile range of deformation. a drastic reduction of the critical minimum stress is observed if the in situ stress gradient is accounted for. This trend can be explained by inspecting the two terms that compose the stress $\sigma_{11}$ (see Fig. 1). That stress depends on the gradient $k_{1}$ and the stress $\sigma_{0}$, which is also the lateral stress at the depth of the interface. In the presence of a stress gradient $k_{1}$, the thicker the overburden. the larger the average stress $\sigma_{1}$. Consequently, the system is destabilized by a smaller value of $\sigma_{01}$ if it has a thick overburden.

the second parameter that enters the definition of the in sim stress in (27) is the stress orientation angle $\rho$. Its influence. which has already been discussed when we compared llow and deformation theories in Fig. 8 , is further explored in Fig. 13. The results of the finite-element andysis are presented for various values of the angle $\rho$ and a constant value of the dimensionless number $\Delta p q H G$. In the compressive regime, depicted in Fig. 13(a), it appears that the critical stress is very much influenced by a value of $p$ in the range of $[0 . \pi ; 4]$ but not so in the interval $[-\pi, 4.0]$. A word of caution, however, is in order before interpeting these results quantiatlively. Inded. 
(a)
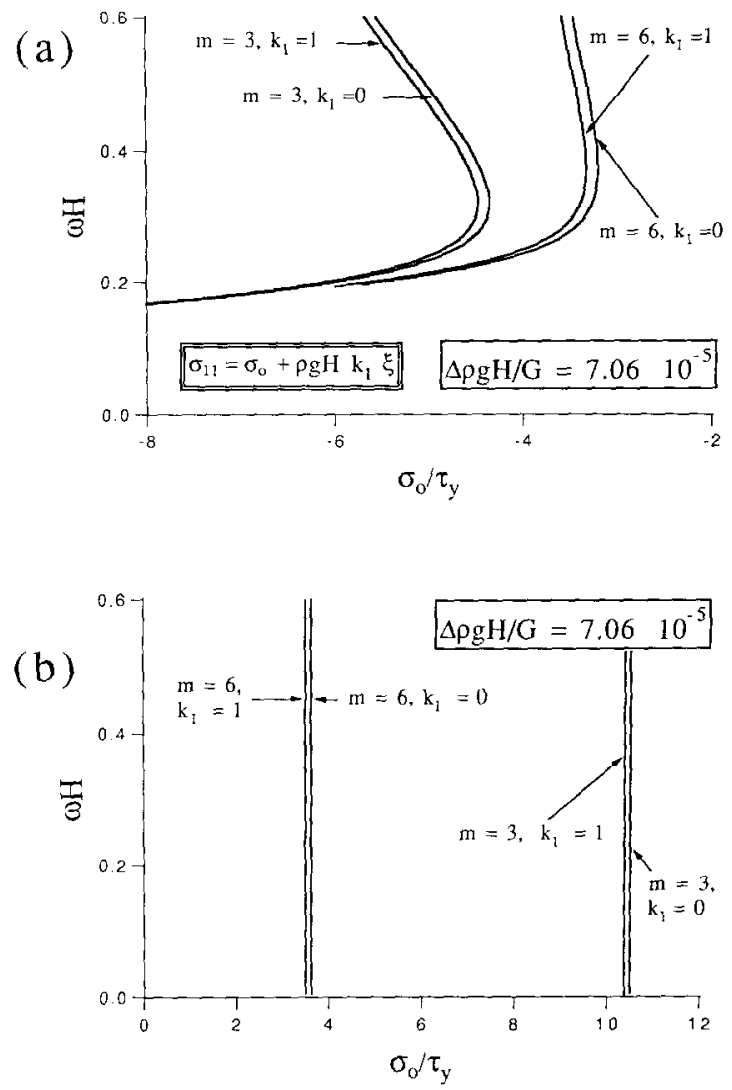

(c)

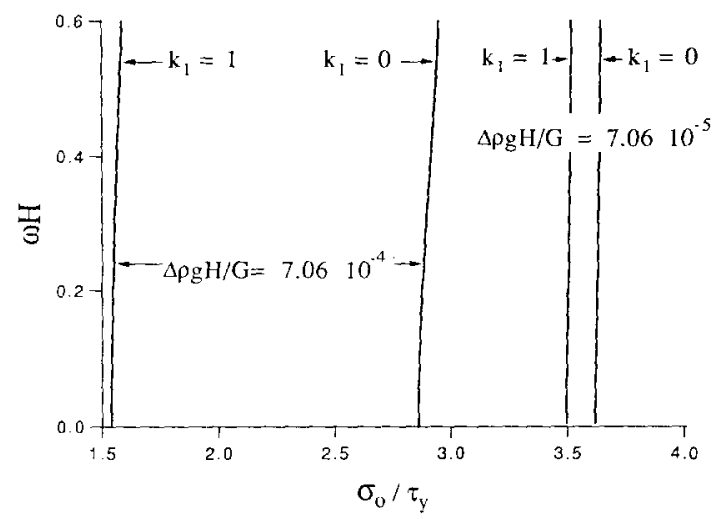

FIG. 12. Influence of the stress gradient parameter $k_{1}$, according to the results of the F. E. M. analysis, in compression for two values of the hardening exponent $m$ (a), in tension for the same value of $m$ (b) and in tension for two values of $\Delta \rho g H / G$ (c). The last graph illustrates the importance of the stress gradient for thick overburdens (a valuc of $10 \mathrm{~km}$ for $H$ corresponds to $\Delta \rho g H G=7.06 \times 10^{-4}$ ). These results are based on deformation theory with a hardening exponent $m=3$ and $\mu=0.3, \beta=0$. 
(a)

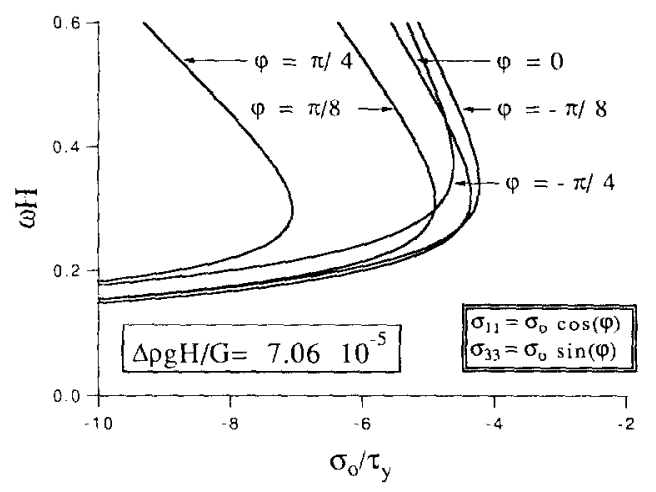

(b)

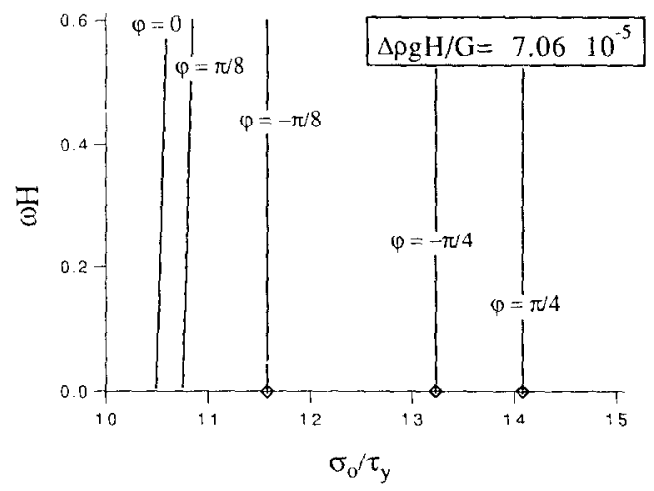

Fici, 13. Influence of the stress orientation angle $\varphi$, aceording to the results of the F.E.M. analysis and for a constant value of $\triangle \rho g H / G$, in compression (a) and in tension (b). In tension, there is no distinction between the stress at loss of ellipticity (represented by diamonds) and the stress at neutral stability, for three of the tive orientation angles studied. The deformation theory was considered with a hardening exponent $m=3$ and $\mu=0.3 . \beta=0$.

it is surprising to find that a compression in the two directions (e.g. $\varphi=\pi / 4$ ) results in a more stable situation than in the case of uniaxial compression $(\varphi=0)$ or in the case of in-plane compression and out-of-plane tension $(\varphi=-\pi / 4)$. This paradoxical result is likely to be an indicator of the limitations of our planar analysis and will only be resolved by a complete 3-D stability analysis. It is with this argument that we invite the reader to reassess with caution the comparison between the predictions of the flowand deformation-theories for varying stress orientation angle which was presented in Fig. 8 .

The last item in our parametric study of the deformation-theory model is the influence of the erosion or redistribution condition at the free surface. This condition was first considered by BIOT and ODE (1965) to account for the crosion of those parts of the top surface that are upheaved and the deposition of the eroded material in the subsided regions. Such a condition leads to a mixed boundary condition between traction rate and displacement rate at the top surface that is expressed by (1) 3 . The influence on stability of this "redistribution" condition, as it was named by Biot and Odé, has already been incorporated in the asymptotic analyses in Sections 3.1-3. The 
(a)

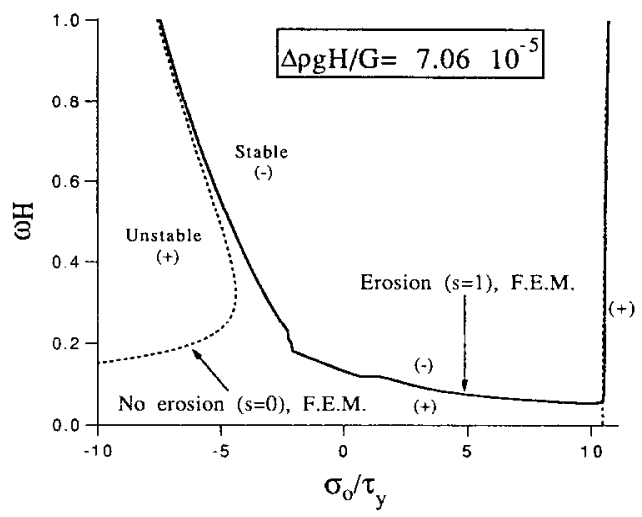

(b)

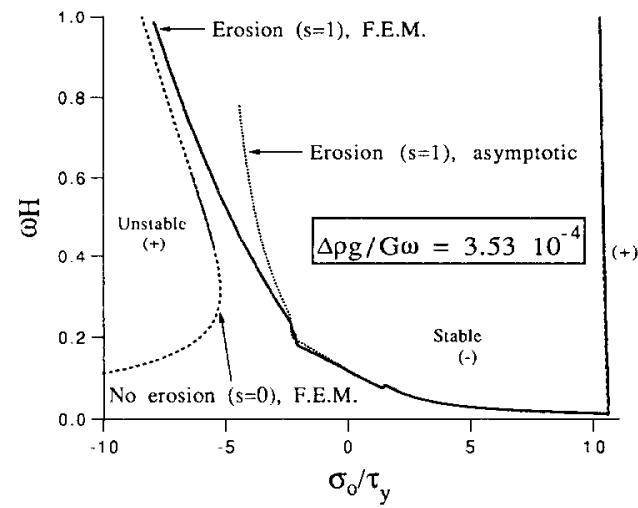

Fig. 14. Influence of erosion for a constant value of $\Delta \rho g H / G$ (a) and $\Delta \rho g / G\left({ }^{\prime}\right)$ (b). The dashed and the solid curves are the results of the F.E.M. analysis in the absence and presence of gravity, respcetivcly. The dotted curve is obtained from the asymptotic analysis for small $\omega H$. Note that the system is unstable, even in the elastic range of deformation, if erosion is accounted for. The deformation theory was considered with a hardening exponent $m=3$ and $\mu=0.3, \beta=0$.

results on the influence of the redistribution condition on the overburden stability, based on finite-element calculations for a constant value of the dimensionless numbers $\Delta \rho g H / G$ and $\Delta \rho H / G \omega$, are depicted in Figs 14(a) and (b), respectively. The new information displayed here is that, with redistribution at the top, the overburden is always unstable for small $\omega H$, exactly as predicted from the asymptotic analysis in Section 3.1. More precisely, if the overburden deforms elastically, the redistribution condition favours the development of perturbalion with wave number $\omega H$ smaller than approximately 0.1 . Note that the critical height ( $\omega H=0.12$ ) for $\sigma_{0}=0$ is the value predicted by (81), which was obtained with the asymptotic analysis for small values of $\omega H$ and with the assumption of an elastic response of the overburden. If a lateral compression or tension is prescribed, the results of the two stability analyses, with and without redistribution at the top, merge for a large enough value of $\omega H$.

To summarize the findings of this last part of the discussion, it is found that the properties of the constitutive model, namely the hardening exponent and cohesion, play an essential role in the stability of the overburden. This influence on stability not 
only affects the minimum critical stresses but can also influence significantly the corresponding mode of instability. Concerning the stress distribution, one can state that the gradient parameter is important only for thick overburdens, whereas the stress orientation angle is important for all thicknesses. The imposition of a redistribution condition at the top surface renders shallow overburdens unstable regardless of the tectonic stress distribution.

\section{CONCLUSIONS}

The am of this work has been to investigate the stability of a prestressed solid layer, composed of a cohesive frictional material, resting atop of a viscoclastic hallspace of lower density. The novel elements introduced in this study pertain to the choices of geometry and material properties for the overburden : it has a finite thickness and is modelled as a strain-rate independent, elastoplastic material. The relaxation time of the substrate introduces a natural characteristic time into the problem, which is used in the stability analysis.

This study is an extension of recent work by TRIANTAFYILIDIS and LFHNIR (1993), who investigated the stability of the interface between the prestressed infinite solid resting atop of a fluid half-space of different density. It was found that because of the siffiness of the solid layer, small perturbations will always have ranges of wavelength for which their amplitude dceays. This leature. coupled with the finite thickness of the overburden. results in the possibility of stable configurations in the present investigation, i.e. configurations for which any small disturbance decays. This behaviour is of course in contrast to all previous analyses of two- or multilayered viscoelastic solid systems based on Biol's approach [see BIOT and ODf (1965) for a review]. These analyses led to the conclusion that a density contrast alone is sufficient to ensure the growth of any perturbation regardless of $i t s$ wavelength. For such viscoelastic models. it was then necessary to compare the rate of growth of the fastest perturbation to the rate of lateral extension or compression of the geological formation to identify stable configurations.

Owing to the existence of stress gradients in the finite overburden. which are inevitable in the presence of gravity, no general analytical solution to the linearized stability problem is possible. A straightforward finite-element technique is thus the numerical method of choice in all the calculations for the resulting eigenvalue problem. An asymptotic analysis for both small and large values of the dimensionless perturbation wave number " $H$ has been performed, where $\theta$ is the perturbation wave number and $H$ the overburden thickness. The small and large values of the dimensionless wave number correspond to beam- and interface-type modes, respectively. These asymptotic analyses provide valuable insight into the behaviour of the system as well as an excellent verification tool for the numerical algorithm. As it turns out, the asymptotic results, together with the analytical solution for the case of zero gravity. provide most of the information required for the range of interest of the parameters in the problem.

The consideration of frictional materials in the present study produces asymmetric stability operators. This asymmetry raises the possibility of complex eigenvalues, 
which correspond to flutter at the point of neutral stability. However, the existence of such instabilities has never been detected in laboratory experiments, nor inferred from field observations. In fact, only real stability exponents are encountered in all the calculations reported here. The reason is to be found in the spectrum of the discretized system's stability matrix. In the absence of friction, the stability operator is symmetric and the stability parameters $\Lambda$ are the two-in general distinct -real eigenvalues of a symmetric $2 \times 2$ matrix. In the presence of friction, the corresponding $2 \times 2$ stability matrix is asymmetric but not sufficiently perturbed from its symmetric counterpart in the absence of friction as to produce complex eigenvalues. For the range of friction coefficients measured in the geological materials of interest, the resulting perturbation of the initially symmetric operator leaves the two eigenvalues $\Lambda$ far enough from each other and hence real.

The main conclusion of this paper is the strong dependence of the stability behaviour of the system on the details of the constitutive model adopted for the overburden. This conclusion is in contrast with the one reached by studying viscoelastic models: there, the geometry of the system and the density contrast determine the stability conditions (BIOT and ODÉ, 1965). We have found that the friction parameter, whose introduction destroys the symmetry of the stability operator, has less influence than the cohesion and hardening of the overburden on the results of the stability analysis. If the hardening or cohesion are decreased, a reduction in the magnitude of the minimum stress for the first instability in both tension and compression is observed. Additionally, the initiation of faulting in extension is favoured and the critical wave number in compression increases. In some instances, this shift in the wave number can be such that interfacial-type instabilities occur at a lower lateral stress magnitude than the beam-mode ones. It is in such cases that the assumption of an infinitely thick top layer, adopted by TRIANTAFYLLIDIS and LFHNER (1993), is justified.

Another consequence of the importance of the constitutive behaviour of the top layer is reflected in the difference between the predictions of the two descriptions of the rate-independent response of solids considered here, namely the flow- and deformation-theory models. Deformation theories used in stability and bifurcation analyses are known to account for the reduced stiffness normal to the proportional loading directions found in rocks, which arises from the presence of randomly oriented cracks or small faults (RUDNICKI and RICE, 1975). The magnitude of the critical stress at the first loss of stability is consistently smaller for deformation than for flow theories, in both the tensile and the compressive range of deformation. Furthermore, concomitance of structural instability and faulting in the tensile range of deformation is predicted by the deformation theory. This last result appears to be in agreement with field observations [see Vendeville and Jackson (1992) for a discussion].

The results of BIOT and ODÉ (1965), concerning the influence of a redistribution condition at the top surface, have been extended here to the case of frictional materials in the overburden. It is found that the erosion of material from uplifted regions and its redeposition in subsiding regions render shallow overburdens always unstable, regardless of the prevailing tectonic stress.

The present results should also contribute to a better understanding of laboratory experiments, conceived as analogue models of geophysical two-layer systems. For example. the role of hardening. as revealed by the results of our stability analysis, 
provides a deeper insight into the interpretation of some of the results obtained recently by SHEMENDA (1992) with a two-layer model of the oceanic lithosphere. It appears from the results presented by Shemenda that buckling is initiated in an elastoplastic layer at a compressive stress that lies below the maximum strength of the analogue material. Furthermore, initiation of faulting is observed only after buckling has stared to develop. These results are similar to the predictions of the present stability analysis.

A second type of analogue test of interest is provided by sand-hox experiments Granular materials have long been recognized as excellent analogue materials for sediments of their ability to develop faults, Early experimental work with granular material to model the initiation of faulting above rising salt domes includes the study of Nettlfion and ElKins (1947). A comprehensive review of the application of sandbox experiments to the study of faulting is found in MANDL's book (1988). In experiments where the deformation is heterogeneous as a result of boundary-condition effects, such as spatially varying sedimentation, the details of the constitutive behaviour of the analogue materials are likely to be unimportant in determining the system's kinematic response and the position of the first set of faults. However, if the heterogeneous deformation results from the development of a structural instability, one should expect the properties of the analogue material to be key parameters in the experiments. For example. we have seen in this study that the existence of a yield vertex was an important aspect of the material description. Sedimentary rocks accommodate part of the deformation by slip along small faults or cracks, justifying the use of a deformation theory in our stability analysis to model the yield vertex. The question remains whether or not analogue materials have a similar feature. It is known that sand deforms in an heterogeneous manner, by isolated local failure prior to maximum load (ARTHLR el al. 1977). Such a prefailure deformation mechanism has been modelled as slip along distributed defects whose growth and interaction were responsible for localization of the deformation and ultimate failure of the specimen (SHI and HORRI, 1989). It remains to be determined whether such a deformation mechanism can result in a plasticity model with a vertex at the loading point on the yield surface. Such questions, concerning the similarity of deformation mechanisms and of nonlinear material properties between analogue and natural materials, could become important once experimentalists are able to determine with sufficient accuracy the stress distribution in the brittle layers of their laboratory experiments. At that time, no doubt, they will try to compare their predictions with the in situ stress conditions in the Earth's crust.

Despite the simplicity of the geometry of our model, only the stability, with respect to small perturbations, of a perfect interface between finite overburden and semiinfinite substratum could be studied. The geological problem of interest presents a number of features, which, although important for an accurate description of the physical problem, have not yet been incorporated in the present study in order to limit its analytical complexity. However, it is clear that the present study should be extended substantially along the following lines.

First, an analysis similar to the one presented here is needed to quantify the influence on stability of the thickness of the substrate. This influence is known to be important for ratios of overburden to substrate thicknesses larger than 0.1 , in the case of 
viscoelastic layered systems (BIOT and ODÉ, 1965). Furthermore, this first extension of the present work is a prerequisite before starting a series of nonlinear stability analyses which are necessary for assessing the validity of the linearized approach used here. This second step of post-instability modelling, obviously to be based on numerical methods in view of the large nonlinear deformations involved, would identify the conditions that control the evolution of the instability towards diapirs (e.g. salt domes), or towards stable finite-amplitude disturbance (e.g. salt pillows). Initiation of faulting in the overburden during the rise of the dome could then also be studied numerically [see LEROY et al. (1989) for further discussion]. Finally, the three-dimensional character of gravitational instabilities will have to be accounted for. A 3-D linearized stability analysis will shed light on the geometry of rising domes that vary from elongated to circular structures, when seen from the top. This 3-D analysis is also required to pursue further the comparison between the initiation of structural instabilities, so far modelled in 2-D, and the initiation of faulting, which has been studied for general 3-D deformation conditions. Although the 3-D stability of certain geological structures can be determined by superposition of 2-D results, as was done some years ago by BIoT (1966), this approach depends on the condition of transverse isotropy with respect to the vertical axis. This condition, of course, is ruined as soon as one allows a differenec between the in situ stress gradients in the two horizontal directions.

\section{ACKNOWLEDGEMENTS}

The authors would like to thank J. L. Urai and D. A. Nieuwland, both at K.S.E.P.L., for helpful comments. The valuable discusions with F. K. Lehner, K.S.E.P.L., and his continual encouragement throughout the realization of this work are gratefully acknowledged. This paper is published by permission of Shell Internationale Research Maatschappij.

\section{REFERENCES}

Arthur, J. R. F., Dunstan, T., Al-ani, Q. A. and Assadi, A. (1977) Plastic deformation and failure in granular media. Géotechnique 27, 53-74.

Bender, C. M. and OrszaG, S. A. (1978) Advanced Mathematical Methods for Scientists and Engineers. McGraw-l Iill, New York.

Bıт, M. A. (1963a) Internal buckling under initial stress in finite elasticity. Proc. $R$. Soc. London A 273, 306-328.

Brot, M. A. (1963b) Surface instability in finite anisotropic elasticity under initial stress. Proc. R. Soc. London A 273, 329-339.

Вют, M. A. (1963c) Interfacial instability in finite elasticity under initial stress. Proc. R. Soc. London A 273, 340-344.

Biot, M. A. (1965) Mechanics of Incremental Deformation. Wiley, New York.

Bıot, M. A. (1966) Three-dimensional gravity instability derived from two-dimensional solutions. Geophysics 31, 153-166.

BIOT, M. A. and ODE, H. (1965) I heory of gravity instability with variable overburden and compaction. Geophysics 30, 213-227.

Chandrasekhar, S. (1961) Hydrodynamic and Hydromagnetic Stability. Oxford University Press, Oxford. 
Chal. K. T. and RUDNick, J. W. (1990) Bilureations of compressible pressure-sensitive materials in plane strain tension and compression. I. Mech. Phys. Solids 38, 875 898.

Danis, Z. F. (1964) Mathematical formulation of salt-dome dynamics. Geophrsics 29, 414 424.

Dorris, J. F. and Nemat-Nassir. S. (1980) Instability of a layer on a half space. J. Appl. Mech. 47,304312 .

Hit.. R. and Hutchisson. J. W. (1975) Bifurcation phenomena in the plane tension test. J. Moch. Phis. Solids 23, 239264.

KRANIZ. R. W. (1991) Measurements of friction coefficients and cohesion for faulting and fault reactivation in laboratory models using sand and sand mixtures. Tectomophysics 188 , $203-207$.

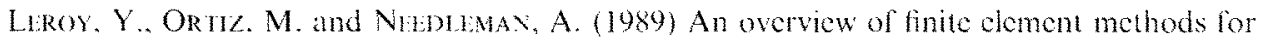
the andysis of strain localization. Proc. Funce U.S. Workshop on Strain Lotedizaton and

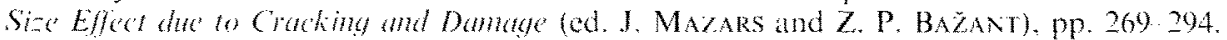
Elsevier. London.

LORET, B. and HaRIRICH:, O. (1991) Acceleration wayes, futter instabilities and stationary discontinuties in inclastic porous media. J. Mech. Phys. Solids 39, 569606.

Mavid, G. (1988) Mechanics of Toctonic Futhing. Elsevier. Amsterdam.

Niliduman. A. (1979) Non-nomality and bifurcation in plane strain tension and compression. J. Mech. Phys. Solide 27, 231254.

Netri:ToN, 1.. L. and Fr.kivs. T. A. (1947) Gcologic models made from granular materials. Am. Gouphis. Linion 28,451 466.

Rambi:Re. H. R. (1981) Grattr. Deformation and the Earth's Chust (second edition). Academic Press. London.

Rambirk, H. R. and Snimanson. O. (1964) Compression of floating clastic and viscous plates affected by gravily, a basis for discussing crustal buckling. Tentomophrsics 1, 101 120.

Ruct. J. R, (1976) The localization of plastic deformation. Theortical and applied Methates. Proc of the 14h, IUTAM Conference (ed, W. T. Komter). pp. 207-220. North Holland. Amsterdam.

Rup.ik Kl. J. W. and Ricf. J. R. (1975) Conditions for the localization of the deformation in pressure-sensitive dilatant materials. I. Mech. Phrs. Solids 23, 371394.

Slitici. F. (1965) A theoretical prediction of salt dome patterns. Geophlisics 30, 633-643.

Shemenda. A. I. (1992) Horizontal lithosphere compression and subduction: constraints provided by physical modeling. J. Goophrs. Re's. 97, 1109711116.

SHI. Z. H. and Horn, H. (1989) Microslip model of strain localization in sand deformation. Moch. Mater. 8, 89.102.

Smolcchonski, M. (1909) Über ein gerrisses Stabilitätsproblem der Elastizitätslehre und dessen Bezichung zur Entsehung von taltengebirgen. Abhandl. Akad. Wiss, Krakau, Math. KI. $1909,3-20$.

Sntt. P. (1986) Bimaterial interlice instabilities in plastic solids. Int. J. Sollds Sthtet. 22, 195 307.

TAYIok. G. 1. (1950) The instability of liquid surfaces when aceelerated in a direction perpendicular to their planes. Proc. R. Soc. Lemton A 201, 192 196.

Triantaryllupis. N. and LiHAir. F. K. (1993) Interfacial instability of density-strattified twolayer systems under initial stress. J. Mech. Phiss. Solids 41, 117-142.

TRIANTAFYLIIDIS, N. and MAKIR, B. N. (1985) On the comparison between microscopic and macroscopic instability mechanisms in a class of fiber-reinfored composites. J. Appl. Mech. 52,794800 .

VeNomitil: B. C, and Jackson, M. P. A. (1992) The rise of dapirs during thin-skinned extension. Marine Petroleum Geol. 9, $331,353$. 